\title{
The following abstracts were presented as posters at the 2015 NEI Psychopharmacology Congress
}

Congratulations to the scientific poster winners of the 2015 NEI Psychopharmacology Congress!

$1^{\text {st }}$ PLACE: Tardive Dyskinesia in the Era of Second Generation Antipsychotics: A Case Report and Literature Review (page 22)

$2^{\text {nd }}$ PLACE: Comparing Deep Brain Stimulation (DBS) and ECT for Treatment Resistant Depression in Elderly Population (page 37)

$3^{\text {rd }}$ PLACE: The Complex Multimodal Management of First-Psychotic Episode in Patients with Morphometric Alteration in Hippocampal Formation (page 30)

\section{Violent Video Games - Physiological and Psychological Impact on Children and Adolescents: Literature Review}

\section{Sree Latha Krishna Jadapalle, $M D^{\prime}$ and Monifa Seawell, $M D^{\prime}$ \\ ${ }^{1}$ Morehouse School of Medicine, Atlanta, GA}

ABSTRACT: Background: Video games became popular in the US in the 1980s. Approximately 145 million Americans play video games regularly and $42 \%$ of households have a video game system. $70 \%$ of children 2 to 18 years old have a game system in their home. In American children, game use increased from an average of 4 hours per week at the end of 1980 s to a current average of 13 hours per week. Recent school shootings involving young perpetrators with history of playing violent video games raised a high alert on further investigating the association between violent video game use and actual violence. American Academy of Pediatrics (AAP) and AACAP proposed that children learn by observing, mimicking and adopting behaviors, which has been the basic principle of social learning model. Literature also supports the idea that prolonged exposure to violent video games can desensitize the youth by emotionally blunting them. Neuro imaging studies so far showed common neural substrates such as the prefrontal cortex and the limbic system being involved in aggression and violent video games exposure.

OBJECTIVES: To understand the physiological and psychological impacts of violent video games on children and adolescents; To understand the neurobiology of aggression and its association with violent video games.

METHODS: Literature review of 12 peer reviewed journal articles dated from 2010 to 2014 was collected, compiled, and analyzed. The key words used for searching databases for articles pertaining to the topic included, but was not limited to, "children, adolescent, heart rate variability, emotion, sleep quality, violent video game, desensitization, aggressiveness".

RESULTS: Literature so far shows an association between daily exposure to violent video games and number of depressive and anxiety symptoms among pre-adolescent youth. The physiological changes observed in the prolonged violent video play group are increased perception of emotional sadness, lower sleep quality and increased alertness during the sleep, higher heart rate and higher low frequency/high frequency (sympathetic predominence) during sleep. There is building evidence that prolonged violent videogame play is significantly related to drastic increases in adolescents' course of aggressive behavior, thoughts and affect; increased physiological arousal; and decreased prosocial (helping) behavior over time. Also, there are studies showing no predictive relationship established between violent video game play and aggressiveness.

CONCLUSION: The results cannot be generalized due to lack of standardized conceptualization, poor methodologies applied, differences in the sample populations and lack of consideration to cultural differences contributing to the social and peer support in some of the studies. The review highlights the further requirement of large controlled prospective studies including diverse ethnicities and also neuroimaging studies in pediatric population to understand the causality.

\section{Lamotrigine for the Treatment of Restless LEG Syndrome}

Vithyalakshmi Selvaraj, $M D^{\prime}$; Babuji R. Gandra, $M B B S^{2}$; Palanikumar Gunasekar, MBBS ${ }^{\prime}$ and Imad Alsakaf, $M D^{\prime}$

${ }^{1}$ Creighton University School of Medicine, Omaha, NE

${ }^{2}$ Essentia Health-Duluth Clinic, Duluth, MN 
ABSTRACT: Lamotrigine is Food and Drug Administration approved for maintenance treatment in bipolar disorder type I, partial and generalized seizures. Restless legs syndrome (RLS) is a neurological condition either idiopathic or secondarily associated with conditions such as iron deficiency, folate deficiency, uremia, pregnancy, Parkinson's disease, diabetes mellitus, and rheumatoid arthritis. The estimated prevalence of RLS is $7 \%$ to $10 \%$. Dopaminergic agents are first-line therapy for RLS although anticonvulsants are increasingly used with success. We present a case of RLS successfully treated with lamotrigine. To the best of our knowledge only two reports of a similar occurrence have been published.

\section{Buprenorphine-Induced Urinary Hesitancy may be Transient and Easy to Treat}

\author{
Anjali Varma, $\mathrm{MD}^{1,2,3,4}$; Mamta Sapra, $M D^{1,2}$ and \\ Joseph Iskandar, $D O^{3}$ \\ ${ }^{1}$ Salem VA Medical Center, Mental Health Clinic, \\ Salem, VA \\ ${ }^{2}$ Virginia Tech Carilion School of Medicine, Roanoke, \\ VA \\ ${ }^{3}$ University of Virginia School of Medicine, \\ Charlottesville, VA \\ ${ }^{4}$ Edward Via College of Osteopathic Medicine, \\ Blacksburg, VA
}

ABSTRACT: Objectives: A retrospective study to examine the prevalence of urinary hesitancy in veterans being treated with buprenorphine/naloxone for opioid dependence and explore the relationship between dose and duration of treatment with these symptoms and other patient and treatment factors.

METHODS: One hundred seventeen charts of veterans with a diagnosis of opioid dependence who have received treatment with buprenorphine since September 2009 to March 2012 in the Buprenorphine Clinic at Salem VA medical center was conducted. The chart review included socio-demographic information, history of opioid addiction, buprenorphine dose, duration of buprenorphine treatment, side effects of treatment, prevalence of urinary hesitancy, duration and treatments used for its management, other prescribed medications, comorbid medical diagnoses and pertinent laboratory results.

RESULTS: Of the 117 charts reviewed, 105 met inclusion criteria for the study. Mean age of subjects was $40.5 \pm$ 12 yrs. $36.5 \%$ of the patients reported no side effects, $22 \%$ patients reported urinary hesitancy. Mean age of subjects who reported urinary symptoms was $39.4 \pm$ 11.2 yrs. Urinary symptoms were reported as early as one day and as late as 2 years after initiation of treatment. Mean timeframe for onset of symptoms was 26 days.
Mean dose of buprenorphine at the time of onset of symptoms was $19.7 \pm 6.6 \mathrm{mg} /$ day. Fifty $(50 \%)$ of the subjects accepted bethanechol for urinary symptoms while the rest increased fluid intake and symptoms subsided over time. Average dose of bethanechol was $25 \mathrm{mg}$ twice a day. $80 \%$ of the patients who did receive bethanechol noted resolution of urinary symptoms after 6 months and were no longer on bethanechol.

CONCLUSIONS: Urinary hesitancy is a known side effect of buprenorphine due to the agonism at the mu opioid receptor leading to detrusor and urinary sphincter muscle contraction. However, this side effect is often under recognized and may go untreated. It may lead to significant discomfort and could possibly interfere with patient compliance with buprenorphine treatment. In most cases the symptoms are mild and transient and can easily be treated with increased fluid intake and use of bethanechol, a cholinergic drug that has long been used for nonobstructive urinary hesitancy or retention, at low doses.

IMPACT: Office based treatment of opioid dependence with buprenorphine/naloxone combination used for maintenance continues to increase among veterans as we fight the opioid epidemic. Since urinary hesitancy is typically an early and transient side effect, patients need to be educated about the symptoms being short lasting and treated as needed with fluids and bethanechol. This is likely to enhance compliance and lower risk of relapse of this highly vulnerable population. Symptoms subsided over time and increased fluid intake in the other $50 \%$ of the patients who declined or decided to defer treatment with bethanechol as noted below.

\section{A Preliminary Investigation of the Influence of the Adverse Side Effects of Medications on Adherence to Medications in Patients with Depression}

Azeez A Lawal

College of Medicine of the University of Lagos, Lagos, Nigeria

ABSTRACT: Depression is a state of low mood and aversion to activity that can affect a person's thoughts, behavior, feelings and sense of well-being. Adherence to medication is a problem in this group of patients because of the symptomatology. It is therefore necessary to know if there are factors that can influence adherence to medication in depressed patients. The objective of the study was to assess the preliminary investigation of the influence of the side effects of medication on adherence to medications in patients with depression in outpatients attending Federal Neuropsychiatric Hospital, Yaba, Lags State. 
Employing Smartphone Apps as Therapy

Extenders in Anxiety, Mood and Cognitive Disorders: The Consumer's Perspective

Dale A. D'Mello, $M D$

Department of Psychiatry, Michigan State University, East Lansing, MI

ABSTRACT: There is wide consensus that the combination of psychopharmacology and cognitive behavior therapy (CBT) is superior to either approach used alone, in managing depressive, anxiety and psychotic disorders. Completing homework assignments is prerequisite for successful outcomes with CBT. Computer-assisted CBT can decrease automatic dysfunctional thoughts. Computer-assisted cognitive enhancement training is effective in patients with schizophrenia and others with mild cognitive impairment. The convergence of digital computer technology and consumer self-empowerment has driven the release of a bewildering array of mental health applications for smartphones and other mobile devices.

OBJECTIVE: The purpose of the present study was to review some of the most popular smartphone apps available for the management of psychiatric disorders.

METHOD: A recent search was conducted of the stores on the iOS and Android platforms, seeking apps for mood, anxiety, and cognitive disorders. Reviews of digital technology resources provided by the International Mental Health Research Organization (IMHRO) at www.psyberguide.org were consulted. Criteria for inclusion included: (1) greater than 10,000 downloads, (2) a score of 3.5 or higher on a consumer 5 star rating scale, (3) acquisition cost less than $\$ 1$, (4) performance tracking capability and (5) messaging capacity. Consumer reviews were scanned.

RESULTS: A total of 12 apps were culled from an expanding universe of thousands. This included the 3 top-rated apps in each of 3 symptom domains: depression, anxiety and cognitive disorders. Ranked in order of popularity (download volume) the leading depression apps were "Depression CBT Self-Help Guide", "eCBT Mood" and "CBT-i Coach". The most popular anxiety apps were "Stop Panic \& Anxiety", "Mindshift" and "Self-Help Anxiety Management (SAM)". The top apps for cognitive enhancement training were "Lumosity", "Elevate Brain Training" and "Fit Brains Focus". Three additional recently introduced apps were included because of their lifesaving potential. "My3" is a suicide prevention app, which enables the user to instantly communicate with family, friends and 911. "Step Away" is designed for managing alcohol use disorder, but can be modified for misuse of any substance. "NAMI Air", which is offered by the National Alliance on Mental Illness, provides information, social support and instant phone access to crisis services. Consumers rated the reviewed apps favorably.

CONCLUSION: Homework assignments, such as relaxation training, cognitive restructuring, behavioral activation are commonly employed as therapy extenders in the management of patients with depression and anxiety. Smartphone apps are achieving wider acceptance in self-management of common psychiatric disorders. Clinicians may need to become familiar with the use of these adjunctive therapeutic tools. Strategies for integrating smartphone apps in routine brief psychopharmacology visits will be reviewed.

\section{Clozapine on Aggression in Pervasive Developmental Disorder - A Case Report and Literature Review}

Rumana Rahmani, $\mathrm{MD}^{\prime}$; Islam Farial, MS-IV'; Pankaj Manocha, $M D^{\prime}$; Omar Colon, $M D^{\prime}$ and Asghar Hossain, $M D^{\prime}$

${ }^{1}$ Bergen Regional Medical Center, Paramus, NJ

EDUCATIONAL OBJECTIVE: To understand the role of clozapine as an effective alternative for aggressive behavior in individuals who failed first line therapy with conventional medications for pervasive development disorder (PDD).

ABSTRACT: PDD is a complex early-onset disorder, which affects multiple developmental domains and severely limits the child's social interaction and/or communication skills. These individuals often engage in repetitive and stereotypical behaviors and/or mannerisms and showcase disruptive behavior. PDD encompasses Autism Spectrum Disorder, Rett's Disorder and Child Disintegrative Disorder. The role of atypical antipsychotics (risperidone, aripiprazole and olanzapine) in addressing disruptive and/or aggressive behaviors in patients diagnosed with PDD has been extensively published. However, due to its adverse effects such as agranulocytosis and seizures, clozapine has not been studied for its diagnostic roles outside schizophrenia treatment. On the contrary, clozapine appears to present with the least number of extra-pyramidal side effects and the World Health Organization list of essential medications considers it as one of the most important medications needed in the healthcare system. We report a case of a 17-year-old male with a past psychiatric history for PDD, Impulse Control Disorder and Obsessive Compulsive Disorder admitted to our hospital, whose impulsivity and aggression successfully improved on clozapine after he 
failed other above mentioned atypical antipsychotic trials. On literature review, a recent study exhibited the role of atypical antipsychotics such as risperidone and aripiprazole as an effective treatment for disruptive symptoms in patients with Autism Spectrum Disorder. However, there remains a subset of patients that appear refractory to treatment to these 2 drugs, where clozapine may serve as a therapeutic option for patients that appear to have failed other atypical antipsychotics. We explain the pharmacology of clozapine and its distinctive effect from other atypical antipsychotics (risperidone and olanzapine) on 5H2A/D2 receptors, resulting in its higher 5H2A/D2 occupancy ratio and relevance for autistic disorder. We also discuss off label use of clozapine in addressing aggressive behavior symptoms in a study involving adolescents with Bipolar disorder, Intermittent Explosive Disorder or Posttraumatic Stress Disorder especially when behavioral dysfunction prevails.

DISCUSSION: PDD severely limits the way a child interacts with society. These behavioral issues such as disruptive behavior can alienate a child and he/she may be considered as being 'violent' and/or a 'menace'. As a reactionary response, the child may internalize these labels that may aggravate his/her aggression. As a psychiatrist, we must recognize the importance of these behavioral issues at an earlier age to effectively facilitate a child's transition to normal functional living within the context of his or her community.

\section{Lorazepam used to Distinguish between}

\section{Catatonia and Delirium: A Case Report and} Review of Literature

Muhammad Zeshan, $\mathrm{MD}^{\prime}$; Cynthia Rutherford, DO, MS; Luisa Gonzalez, MD, FAPA and Panagiota Korenis, $M D$

${ }^{1}$ Albert Einstein College of Medicine, Bronx NY

ABSTRACT: Subject Objective: Catatonic syndromes involve a complex mixture of motor, behavioral, and systemic manifestations that are derived from unclear mechanisms. Catatonia usually occurs in psychiatric and general medical conditions. It is most commonly associated with mood disorders, specifically bipolar I disorder. Diagnosing catatonia at initial evaluation is important because the syndrome may go unrecognized which often complicates the management. In general, patients with catatonia present with immobility, rigidity, mutism and posturing. While the pathophysiology of catatonia remains unclear, the literature suggests involvement of numerous neurotransmitters which include: GABA, dopamine and glutamate. Treatment for catatonia varies depending on the underlying mechanism and involves medications that target the specific neurotransmitter.
METHOD: Here we present the case of a 51 year-old male with bipolar I disorder, current episode depressed who was brought to our Comprehensive Psychiatric Emergency Program (CPEP) with sudden changes in his behavior. He was anorexic, anhedonic and avolitional. Upon initial examination, the treatment team noted that he had a clouded sensorium and diagnosis of delirium was considered; however, there were no overt signs of delirium. Based on the patient's presentation the team considered a diagnosis of catatonia and ordered lorazepam 1mg intramuscular injection.

RESULTS: The patient was reassessed one hour after administration of lorazepam. His sensorium cleared and his mental status was vastly improved.

CONCLUSION: We use this case to clarify challenges when working with apparently depressed patients with altered mental status in the emergency setting. We aim to illustrate some treatment options based on the underlying mechanisms of catatonia.

\section{Lurasidone for the Treatment of Major Depressive Disorder with Mixed Features: A Randomized, Double-Blind, Placebo-Controlled 6 Week Trial}

Trisha Suppes, $M D^{1,2}$; Robert Silva, PhD ${ }^{3}$; Yongcai Mao, PhD ; Josephine Cucchiaro, PhD ${ }^{3}$; Caroline Streicher, BA ${ }^{3}$; Andrei Pikalov, $M D, P h D^{3}$ and Antony Loebel, $M D^{3}$

${ }^{1}$ Stanford School of Medicine, Stanford

${ }^{2}$ VA Palo Alto Health Care System, Palo Alto, CA

${ }^{3}$ Sunovion Pharmaceuticals Inc., Fort Lee, NJ and

Marlborough, MA

OBJECTIVE: Accumulating evidence indicates that manic symptoms, below the threshold for hypomania (mixed features), are common in individuals with major depressive disorder. This form of depression is often severe, and is associated with an increased risk for recurrence, suicide attempts, substance abuse, and functional disability. This study evaluated the efficacy and safety of lurasidone in major depressive disorder with mixed (subthreshold hypomanic) features.

METHODS: Patients with MDD, presenting with 2 or 3 DSM-5 mixed features criteria manic symptoms on most days over $\geq 2$ weeks prior to screening were randomized to 6 weeks of double-blind treatment with either lurasidone $20-60 \mathrm{mg} / \mathrm{d}$ or placebo. Changes from baseline in Montgomery-Asberg Depression Rating Scale (MADRS) total score (primary assessment) and Clinical Global Impression, Severity scale (CGI-S) score (key secondary), and the Young Mania Rating Scale (YMRS) were analyzed using a mixed model for repeated 
measures analysis. Responder rates $(\geq 50 \%$ reduction from baseline in MADRS total score) were analyzed using logistic regression.

RESULTS: Treatment with lurasidone $(\mathrm{N}=108)$ was associated with significantly greater improvement vs placebo $(\mathrm{N}=100)$ from Weeks 2 through 6 on both the MADRS and CGI-S. At Week 6, LS mean change for lurasidone vs placebo on the MADRS total score was -20.5 vs $-13.0(\mathrm{P}<0.001$; effect size, 0.80), and on the CGI-S score was -1.83 vs $-1.18(\mathrm{P}<0.001$; effect size, 0.60). Week 6 responder rates, for lurasidone vs placebo, were $67.6 \% \quad$ vs $\quad 33.0 \% \quad(\mathrm{P}<0.001 ; \quad \mathrm{NNT}=3)$. Significant improvement in manic symptoms, assessed by the YMRS, was also observed $(\mathrm{P}<0.001$; effect size, 0.61$)$, in addition to other secondary efficacy endpoints. Discontinuation due to adverse events was low; the most common adverse events (incidence of lurasidone $>$ placebo) were nausea (6.4\% vs. $2.0 \%)$ and somnolence (5.5\% vs. $1.0 \%)$.

CONCLUSIONS: Lurasidone was effective and well tolerated in this study involving patients with MDD associated with subthreshold hypomanic symptoms (mixed features).

FUNDING: This study was funded by Sunovion Pharmaceuticals Inc.

Clinicaltrials.gov identifier: NCT01423240.

\section{Long-Term use of Lurasidone in Patients with Bipolar Disorder: Safety and Effectiveness Over 2 Years of Treatment}

Andrei Pikalov, MD, PhD'; Joyce Tsai, PhD'; Yongcai Mao, PhD'; Robert Silva, PhD'; Josephine Cucchiaro, $P h D^{\prime}$ and Antony Loebel, $M D^{\prime}$

${ }^{1}$ Sunovion Pharmaceuticals Inc., Fort Lee, NJ and Marlborough, MA

OBJECTIVE: Among patients with a diagnosis of bipolar disorder, episodes typically recur over many decades, with periods of depression occurring with greater frequency than episodes of mania. Few studies are available that provide evidence for the acute efficacy of atypical antipsychotics, administered as monotherapy, or adjunctively with mood stabilizers, for the acute or maintenance therapy of bipolar depression. Thus, there is clearly a need for additional options that are safe, welltolerated, and effective in bipolar patients at risk for depression recurrence. The aim of the current study was to evaluate the safety and effectiveness of lurasidone over 2 years of treatment in bipolar depression.

METHODS: Patients with bipolar I depression were enrolled in one of three 6-week, double-blind, placebo-controlled trials (monotherapy with lurasidone, 1 study; adjunctive therapy with lurasidone and lithium or valproate, 2 studies); followed by 6 -month open-label extension treatment, with study completers treated for an additional 18 months with lurasidone in the dose range of $20-80 \mathrm{mg}$. The Clinical Global Impression-Severity (CGI-S) scale was included as a measure of treatment efficacy.

RESULTS: A total of 122 patients entered the 18-month continuation study after completing 6 weeks of doubleblind treatment, followed 6 months of extension treatment. Overall, $19.7 \%$ of patients discontinued during the 18 month study period, including $6.6 \%$ due to adverse events and $1.6 \%$ due to insufficient efficacy. An additional 58 patients $(47.5 \%)$ were ongoing at the time the study was terminated by the sponsor. Among patients who entered the 18-month continuation study, the mean CGI-S score at baseline of the acute study was 4.3 , which improved to 2.8 at baseline of the 6-month extension study, and showed further improvement to a mean of 2.1 at baseline of the 18-month continuation study. At 18-month endpoint, the mean CGI-S score was 1.7 (observed case [OC] analysis; LOCF, 1.9). Mean change in weight, from acute baseline to 18-month continuation endpoint was $+0.8 \mathrm{~kg}(\mathrm{OC}, \mathrm{n}=55)$; median change in cholesterol was $-3.0 \mathrm{mg} / \mathrm{dL}$, and median change in triglycerides was $+26.0 \mathrm{mg} / \mathrm{dL}(\mathrm{OC}$, $\mathrm{n}=54$ for both).

CONCLUSIONS: Up to 2 years of treatment with lurasidone was safe and well-tolerated in this bipolar depression population, with minimal effects on weight and metabolic parameters. Efficacy was maintained during extended treatment with lurasidone.

FUNDING: This study was funded by Sunovion Pharmaceuticals Inc.

Clinicaltrials.gov identifier: NCT00868452; NCT00868699; NCT01284517; NCT00868959; NCT01485640.

\section{Optimizing Response to Lurasidone in Patients} with Acute Schizophrenia: A Randomized, Double-Blind, Placebo-Controlled Study of Dosing Regimens

Antony Loebel, MD'; Robert Silva, PhD'; Robert Goldman, PhD'; Kei Watabe, $M S^{\prime}$; Andrei Pikalov, MD, PhD'; Josephine Cucchiaro, $P h D^{\prime}$ and John M. Kane Ka,3 $^{2,3}$

${ }^{1}$ Sunovion Pharmaceuticals Inc.

${ }^{2}$ Zucker Hillside Hospital, Glen Oaks, NY

${ }^{3}$ Hofstra North Shore-LIJ School of Medicine, Glen

Oaks, NY

STUDY OBJECTIVE: To evaluate the efficacy and safety of low-dose lurasidone in patients with schizophrenia and 
the effect of dose increase in patients demonstrating inadequate response after 2 weeks of standard lurasidone dosing.

METHODS: In this 6-week, randomized, double-blind, placebo-controlled study, patients experiencing an acute exacerbation of schizophrenia were initially randomized in a 1:2:1 ratio to receive lurasidone $20 \mathrm{mg} / \mathrm{d}$, lurasidone $80 \mathrm{mg} / \mathrm{d}$, or placebo. After 2 weeks of treatment, patients in the lurasidone $80 \mathrm{mg} / \mathrm{d}$ group classified as early nonresponders (defined as $<20 \%$ improvement on the Positive and Negative Syndrome Scale [PANSS] total score) were re-randomized in a $1: 1$ ratio to receive lurasidone $80 \mathrm{mg} / \mathrm{d}$ or $160 \mathrm{mg} / \mathrm{d}$ for the remainder of the study.

RESULTS: The intent-to-treat analysis included 411 patients randomized to receive lurasidone $20 \mathrm{mg} / \mathrm{d}$ ( $\mathrm{n}=101)$, lurasidone $80 \mathrm{mg} / \mathrm{d}(\mathrm{n}=198)$, or placebo ( $\mathrm{n}=112$ ). At Week 6, mean change from baseline in the PANSS total score was not significantly different for lurasidone $20 \mathrm{mg} / \mathrm{d}$ compared with placebo (-17.6 vs -14.5; $\mathrm{P}=0.26)$. Assay sensitivity for the study was confirmed by the patient group receiving lurasidone 80 to $160 \mathrm{mg} / \mathrm{d}$, which demonstrated significant improvement in the PANSS total score relative to placebo (mean change from baseline, $-24.9 ; \mathrm{P}<0.001$; effect size $=$ 0.63). In early responders to lurasidone $80 \mathrm{mg} / \mathrm{d}$ $(\mathrm{n}=100)$, mean PANSS total score was significantly reduced compared with the placebo group at Week 2 $(-20.4$ vs $-8.2 ; \mathrm{P}<0.001)$ and Week 6 study endpoint $(-32.1$ vs $-14.5 ; \mathrm{P}<0.001$; effect size $=1.06)$. In early nonresponders to lurasidone $80 \mathrm{mg} / \mathrm{d}(\mathrm{n}=95)$, the reduction in mean PANSS total score from Week 2 to Week 6 was significantly greater with dose escalation to lurasidone $160 \mathrm{mg} / \mathrm{d}(\mathrm{n}=43)$ compared with continuation of the $80 \mathrm{mg} / \mathrm{d}$ dose $(\mathrm{n}=52 ;-16.6$ vs -8.9 ; $\mathrm{P}=0.023$; effect size $=0.52$ ). The most common adverse events (AEs) associated with lurasidone therapy (dose groups combined vs placebo) were akathisia $(8.7 \%$ vs $1.8 \%)$, nausea $(6.4 \%$ vs $3.6 \%)$, and vomiting $(3.7 \%$ vs $0.9 \%)$. The rate of study discontinuation due to AEs was higher in the placebo group $(7.1 \%)$ than in lurasidone 20 $\mathrm{mg} / \mathrm{d}(2.0 \%)$ or lurasidone 80 to $160 \mathrm{mg} / \mathrm{d}(4.0 \%)$ groups.

CONCLUSION: For patients not responding to 2 weeks of treatment with lurasidone $80 \mathrm{mg} / \mathrm{d}$, dose increase to lurasidone $160 \mathrm{mg} / \mathrm{d}$ provided statistically significant and clinically meaningful improvement compared with continuing lurasidone $80 \mathrm{mg} / \mathrm{d}$. Lurasidone $20 \mathrm{mg} / \mathrm{d}$ was not efficacious in the treatment of patients with schizophrenia.

FUNDING: This study was sponsored by Sunovion Pharmaceuticals Inc.
ClinicalTrials.gov identifier: NCT01821378.

REFERENCES:

Loebel A, Silva R, Goldman R, Watabe K, Cucchiaro J, Kane J. Optimizing treatment with lurasidone in patients with schizophrenia: results of a randomized, double-blind, placebo-controlled trial (OPTIMIZE trial). Presented at the Annual Meeting of the American College of Neuropsychopharmacology; December 7-11, 2014; Phoenix, AZ.

\section{Major Depressive Disorder with Mixed Features: Clinical Characteristics of Patients Entered in A Multiregional Placebo-controlled Study}

J. Cara Pendergrass, PhD'; Steven Targum, $M D^{\prime}$; Trisha Suppes, $\mathrm{MD}, \mathrm{PhD}^{2,3}$; Sang Lee ${ }^{1}$; Robert Silva, PhD ${ }^{4}$; Josephine Cucchiaro, $\mathrm{PhD}^{4}$ and Antony Loebel, $\mathrm{MD}^{4}$

${ }^{1}$ Clintara LLC

${ }^{2}$ Stanford School of Medicine, Stanford University, Stanford, CA

${ }^{3}$ VA Palo Alto Health Care System, Palo Alto, CA

${ }^{4}$ Sunovion Pharmaceuticals Inc.

STUDY OBJECTIVE: Major depressive disorder (MDD) with mixed features (subthreshold hypomania) has been identified as a distinct nosological entity in the DSM-5. We identified the predominant manic symptoms at baseline in a multiregional, placebo-controlled study involving patients with MDD with mixed features.

METHODS: This study was conducted as part of the patient selection process for RESOLVE 1, a randomized, 6-week, double-blind, placebo-controlled, flexible-dose, parallelgroup study of lurasidone (once-daily, flexibly dosed 20-60 mg/d) for the treatment of MDD with mixed features. Patients had a diagnosis of MDD, current major depressive episode, and 2 to 3 protocol-specified manic symptoms (consistent with DSM-5 criteria) for $\geq 2$ weeks prior to screening. Forty-four sites in Russia, Serbia, Ukraine, the United Kingdom, and the United States participated in the study between April 2011 and October 2014.

RESULTS: A total of 211 patients were enrolled in the study ( $\mathrm{n}=62$, United States; $\mathrm{n}=62$, Ukraine; $\mathrm{n}=52$, Serbia; $\mathrm{n}=31$, Russia; $\mathrm{n}=4$, United Kingdom). Baseline mean \pm standard deviation (SD) MontgomeryÅsberg Rating Scale (MADRS) score was 33.2 \pm 4.2 , and the mean \pm SD Hamilton Anxiety Rating Scale score was $16.9 \pm 6.4$, with no significant differences in scores in the United States compared to Europe. The MADRS items of apparent sadness, reported sadness, reduced sleep, and concentration difficulties were associated with the highest mean item scores at study baseline (4.1 \pm $0.8,4.4 \pm 0.7,4.3 \pm 1.1$, and $4.0 \pm 0.9$, respectively) Baseline mean Young Mania Rating Scale (YMRS) score 
was $12.4 \pm 5.0$ in the United States and $10.0 \pm 4.1$ in Europe $(\mathrm{t}=3.56 ; \mathrm{P}=0.0004)$. Overall, the YMRS items of decreased need for sleep, increased rate or amount of speech (talkativeness), and irritability were associated with the highest mean item scores at study baseline $(2.0 \pm 0.9,2.1 \pm 2.1$, and $1.9 \pm 1.4$, respectively). Of the 211 patients, $133(63.0 \%)$ endorsed experiencing 2 of the 7 protocol-specific manic symptoms for $\geq 2$ weeks prior to screening, whereas 78 patients $(37.0 \%)$ endorsed 3 manic symptoms. Flight of ideas (racing thoughts) and increased talkativeness (pressured speech) were endorsed by $>60 \%$ of patients.

CONCLUSION: We identified flight of ideas (racing thoughts) and increased talkativeness (pressured speech) as the most common manic symptoms meeting eligibility criteria in this acutely depressed population. In this analysis, irritability and distractibility (concentration difficulties) were also commonly reported.

FUNDING: This study was sponsored by Sunovion Pharmaceuticals Inc.

ClinicalTrials.gov identifier: NCT01421134.

\section{REFERENCES:}

Pendergrass JC, Targum S, Suppes T, et al. Major depressive disorder with mixed features: clinical characteristics of patients entered in a multiregional placebo-controlled study. Presented at the annual meeting of the American Society of Clinical Psychopharmacology (ASCP); June 22-25, 2015; Miami, Florida.

\section{Mental Health Nurse Practitioners Embracing Telepsychiatry}

Christina Fuchs, MSN, PMHNP-S' and Marie Tamanji, $M S N, P M H N P-S^{\prime}$

${ }^{1}$ Alverno College, Milwaukee, WI

ABSTRACT: Purpose: This multidimensional capstone scholarship delivers a thoughtful investigation of telepsychiatry service. Over a one year time span, a comparison is analyzed capturing the intervention of telepsychiatry services compared to traditional in-person psychiatric services delivered to adults suffering from mental illness in rural underserved areas. The emerging role of the PMHNP is blended in utilizing the Clinical Scholar Model to support the telepsychiatry process change.

CONTENT: The nation-wide shortage of psychiatric providers is igniting innovative ideas for delivering mental health treatment. Telepsychiatry captures the advantages of modern technology, connecting providers to those "hard to reach" patients and families around the globe. PMHNPs steeped in scientific scholarship, committed to quality care, and possessing leadership qualities, are well-suited for implementing a successful telepsychiatry process change.

METHODOLOGY: Capturing the spirit of change, the Clinical Scholar Model can assist with implementing the telepsychiatry process utilizing the PMHNP role. Workshops developed could begin with promoting the spirit of inquiry through reflection and observation of community and organization needs, analysis and scientific critique of data, synthesis of evidence based peerreviewed articles, and application of telepsychiatry to a small sample size with evaluation of patient outcomes, and finally dissemination to community stake-holders.

POPULATION: For purposes of the capstone project, the population included adults aged 18-65 living in rural communities with chronic mental illness, according to the DSM-IV-TR.

RESULTS: Across the articles selected and small sample size in rural Wisconsin, commonalities were found. Telepsychiatry allows for the patient to remain in their community, receiving prompt and available mental health care with reduced costs, travel time and lost work time. Organizations using telepsychiatry had a reduction in provider cost, with less travel and lodging cost for the provider, and increased provider access. Empowerment to the patient and community was chiefly recognized when allowing the choice of telepsychiatry or traditional in person appointments.

CONCLUSION: Satisfaction survey indicated overall an equivalency between traditional in person appointments and telepsychiatry. Patients in Taylor County, WI, provided positive reviews of the telepsychiatry process and technology. An electronic medical record system supports their telepsychiatry process, and allows for monitoring of clinical quality outcomes, including tracking psychiatric medication trials, BMI, laboratory data, and appointments. During an interview with their telepsychiatry provider, he revealed the unexpected benefits of removing barriers, mental health care stigma and enhancing transparent patient care.

\section{Penultimate Symptoms of Truman Show Syndrome: Reduplicative Paramnesia with Prop Delusions - A Case Study}

\footnotetext{
Mohamed Salah'; Dima Mohammad ${ }^{2}$; Tasnim Mohammad ${ }^{2}$ and Alan R. Hirsch, $M D^{3}$

${ }^{1}$ St. James School of Medicine, Kralendijk-Bonaire, Netherlands Antilles

${ }^{2}$ Caribbean Medical University, Willemstad, Curaçao

${ }^{3}$ Smell \& Taste Treatment and Research Foundation, Chicago, IL
} 
OBJECTIVES: To highlight the insensate delusions of Truman Show Syndrome.

METHODS: Case Study: 20 year old right-handed, single, African American male complains of, since the age of 7, feeling like he is the central character in the movie The Truman Show, where everyone is watching him and everyone (except immediate family) have been replaced by actors. Moreover, the physical objects around him have been replaced by artificial cardboard movie scenery. He repetitively touches surrounding furniture and objects in order to validate their true nature. He believes that the physical environment surrounding him is fake, as if "in an experiment, or in the movie The Matrix". When estivating and flying to a new city, he perceives the fellow travelers, flight attendants, and pilots as actors and the plane and city itself are all elaborate props in his personal drama. He admits ideas of references, whereby glances from strangers suggest to him that he is being watched as the protagonist of this elaborate scheme. As a result, he avoids social gatherings and groups. Confiding in his parents received reassurance. Querying his close companions as to their occupation as actors is pleasantly rebuffed as if it is humorous. When the monothematic delusions are present, he feels overwhelmed saying "if the delusional thought of being the star of the Truman Show enters my thought process, I can not derail my own thought train".

Approximately $70 \%$ of the time, he has insight as to the delusional nature of his condition and $30 \%$ of the time he is submerged in the pathological belief system. Intensity of the delusion is enhanced by lack of sleep and markedly exacerbated by marijuana, 3,4-methylenedioxyamphetamine (MDA), and psilocybin mushrooms.

He affirms occasional auditory hypnogogic hallucinations as well as when surrounded by a large crowd, at which time he notes nondescript noises and his name being called in a mechanical voice. His past medical history is relevant for head trauma with loss of consciousness at age 9 and recurrent syncope while playing a thoracic compression game, "Pass Out Challenge", with friends.

RESULTS: Birth, developmental, family, social history, and general physical exam are normal.

Normal neurologic exam includes gait, cerebellar, sensory, \& reflexes.

-Abnormalities: Motor Exam: Right Pronator Drift.

-Ancillary Tests include: Clock drawing test 4/4; Semantic Fluency Test: 33; Beck Depression Inventory type II: 29; Beck Anxiety Inventory: 16; Alcohol Sniff Test: 17

TREATMENT: Despite Aripiprazole, he did not follow up with the presumption that the medical team was also included in his delusion.
CONCLUSION: Unlike other misidentification delusional syndromes like Capgras or Fergoli's, not only are the people replaced with 'imposters' but inanimate objects are also incorporated in the Truman Show delusion. Queries should be conducted as to the extent of the delusion systems in those who present with this syndrome.

\section{The Insight of "Inside Out" - Co-Localization of Color and Affect; Feel Blue See Blue, Feel Happy See Yellow}

\author{
Mohamed Salah'; Tasnim Mohammad ${ }^{2}$; Dima Moham- \\ $\mathrm{mad}^{2}$; Alan R. Hirsch, $\mathrm{MD}^{3}$ and Neil Allen, $M D^{4}$ \\ ${ }^{1}$ St. James School of Medicine, Kralendijk-Bonaire, \\ Netherlands Antilles \\ ${ }^{2}$ Caribbean Medical University, Willemstad, Curaçao \\ ${ }^{3}$ Smell \& Taste Treatment and Research Foundation, \\ Chicago, IL \\ ${ }^{4}$ Consultants in Neurology, Northbrook, IL
}

INTRODUCTION: Neuroanatomical localization of color is in the occipital lobe, rather than the limbic system. Colloquialisms bind colors with emotions, as illustrated by the euphemisms of 'feeling blue' in states of depression and 'golden' when happy. Zeitgeist cements this connection as manifest in the happiness of the color yellow in McDonalds golden arches, euphoric Big Bird, and ecstatic Spongebob, in contrast to the unhappiness of the blue grumpy Cookie Monster in 'Sesame Street' and a melancholy Eeyore from "Winnie the Pooh".

Such associations suggest an intrinsic link between these colors and emotions. This would imply the existence of either a second anatomic localization of these colors exists in the limbic system or a well-established pathway from Papez circuit connecting these emotions and the occipital areas for these colors. A clinical case of deep brain stimulation (DBS)-induced co-occurrence of these specific colors and emotion is presented.

METHODS: Case Study: 25 year old right handed woman for intractable obsessive compulsive disorder (OCD) had electrodes implanted bilaterally in the anterior limb of the internal capsule, just anterior to the rostral border of the anterior commissure, for induction of DBS for OCD management. Postoperatively, in the process of calibration of the electrodes, the patient experienced $\approx 100$ epochs of stereotypic phenomena during subsequent calibration visits. These episodes lasted for a second, consisting of a colored haze, an egodystonic mood, and a taste followed by a vivid nostalgic autobiographical flashback with an olfactory hallucination.

These episodes were dichotomized into two distinct types: Yellow and Blue. During the Yellow event she 
would see a translucent yellow-colored tint obscuring her vision and a lemon in her upper left visual field, experience intense euphoria, and taste lemon. She would then experience a happy nostalgic memory and smell. In the Blue event, she would see a transparent bluecolor obscuring her vision, envision blueberries in her lower right visual field, taste blueberries, feel depressed, and recall a sad memory and associated smell. Prior to these incidents, the patient had never experienced hallucinations, phantogeusia, nor phantosmia; however she did admit to odor-induced migraines and aversions.

RESULTS: Chemosensory tests:

Olfactory: Normal: Alcohol Sniff Test (14); Pocket Smell Identification Test (3); University of Pennsylvania Smell Identification Test (38). Abnormal: Snap \& Sniff Phenylethanolamine Threshold: Left $=-5.0$. Right $=-3.0$ (Hyposmia).

Retronasal Smell: Normal Jelly Bean Difference (9). Gustatory: Normal Propylthiouracil Disc (10).

DISCUSSION: It has been hypothesized that DBS current spreads and stimulates olfactory, gustatory, and affective pathways ("What's in a "Smile?"”, Okun 2004). Similarly, diffusion to the visual pathways may explain the color co-occurrence. Such diffusion may also explain the physiology of synesthesia.

\section{Genetic Polymorphisms and Antidepressant Adverse Effects}

Rajnish Mago, $\mathrm{MD}^{\prime}$; Sandeep Gupta, $\mathrm{MD}^{2}$; Kelly Huhn, BS ; Ronakkumar Shah, MBBS ${ }^{2}$; Rachel Scott, PharmD ${ }^{3}$; Daniel Dowd, PharmD ${ }^{3}$ and Lorna Khensouvann, $M S^{3}$

${ }^{1}$ Mood Disorders Program, Thomas Jefferson University, Philadelphia, PA

${ }^{2}$ Christiana Care Health System, Newark, DE

${ }^{3}$ Genomind, Inc

OBJECTIVES: To assess the association between antidepressant adverse events (AEs) or poor response and genetic polymorphisms including CYP450 polymorphisms that affect antidepressant metabolism.

METHODS: This case-control study assessed the association between increased AEs to antidepressants (Cases) and poor response without significant AEs (Controls). For Cases, increased AEs were defined as $\geq 3$ moderate/ severe or $\geq 5$ mild AEs on a usual dose. Controls were patients with $<30 \%$ reduction in depression and minimal or no AEs following antidepressant treatment. DNA samples were analyzed using the Genecept Assay (psychiatric genetic test) and SPSS v.19 was used for statistical analyses (Fisher's Exact test).
RESULTS: This preliminary analysis included 50 subjects (21 Cases, 29 Controls). 57.1\% of Cases were poor/ intermediate metabolizers (PM/IMs) for the relevant P450 enzyme vs. $17.2 \%$ of Controls $(p=0.006)$. Of those who had $>1$ severe AE, $52.9 \%$ were found to be PM/IMs $(p=.061)$ as were $69.3 \%$ of those who had had $>2$ severe AEs $(p=0.005)$. $27.6 \%$ of Controls were ultrarapid metabolizers (UMs) of the relevant enzyme vs. $14.3 \%$ of Cases $(p=0.221)$. MTHFR and SLC6A4 genotypes were evaluated, but did not reach statistical significance in this sample size.

CONCLUSIONS: Patients on selected antidepressants who had increased AEs were much more likely to be PM/IMs of the P450 enzyme responsible for metabolism of the selected antidepressant(s). This prevalence was even higher in patients who had two or more severe AE. Genetic testing should be routine in these patients. Patients with nonresponse to antidepressants were numerically more likely to be UMs on the relevant isoenzyme but this was not statistically significant in this sample. Recruitment in this study continues to evaluate these correlations in a larger sample.

FUNDING: This study was funded by Genomind, Inc.

\section{Pharmacogenetics: Guiding Treatment, Improving Care}

\section{Elizabeth Drew, $M D^{1,2}$ and Patricia Allen, MSN, $P M H N P-B C^{\prime}$}

${ }^{1}$ Summit Behavioral Health, Doylestown, PA

${ }^{2}$ Addiction Medical Treatment Specialty, Private addiction practice

ABSTRACT: More than $65 \%$ of those with substance use disorders also suffer from a mood or anxiety disorder. For the past decade pharmacogenetics has emerged as a promising clinical tool for the treatment of those with co-occurring disorders. This testing enables individualized treatment and offers the client and practitioner valuable information that can positively impact the course of treatment. Many of our clients come to us with unresolved or worsening symptoms, a history of treatment non-compliance, or premature discontinuation of prescribed medications due to the medication not working or intolerable side effects. Genetic testing guides the prescriber, individualizes care, educates the client, and facilitates a clinical partnership that empowers the client and supports recovery. With the advances in medication assisted treatment for addictions, genetics testing can helps us work with our patients to design the most successful recovery program. When patients have appropriate treatment from the beginning, they are more likely to remain in treatment and recovery. Current use of 
genetic testing is helping us to achieve retention rates up to $80 \%$ in our treatment programs. Case studies demonstrate the clinical value of genetic testing and its impact on clinical outcomes for clients with co-occurring disorders, and also reduce the stigma that is a barrier to treatment.

\section{Prior Hospitalization and Treatment Response in Schizophrenia: A Moderator Analysis}

Antony Loebel, $\mathrm{MD}^{\prime}$; Cynthia Siu, PhD'; Michael Tocco, PhD'; Josephine Cucchiaro, PhD'; Andrei Pikalov, $\mathrm{MD}, \mathrm{PhD}^{\prime}$ and Steven Potkin, $\mathrm{MD}^{3}$

${ }^{1}$ Sunovion Pharmaceuticals Inc., Fort Lee, NJ
${ }^{2}$ COS \& Associates Ltd., Central, Hong Kong
${ }^{3}$ University of California, Irvine, Irvine, CA

STUDY OBJECTIVES: Responsiveness to treatment has been associated with stages of illness in schizophrenia, with greater treatment response at earlier stages of illness. The objective of this study was to assess if diminished treatment responsiveness is associated with relapse and the number of prior hospitalizations in schizophrenia.

METHOD: A post-hoc analysis was performed on pooled data from three 6-week, double-blind, placebocontrolled trials for which prior hospitalization and PANSS (LOCF) data were available. ANCOVA model was applied to compare mean changes from baseline in PANSS total among patients with and without prior hospitalization.

RESULTS: Baseline PANSS total was comparable between the lurasidone $(96.8, \mathrm{n}=844)$ and the placebo $(96.4$, $\mathrm{n}=358$ ) groups. In patients who had no prior hospitalization ( $\mathrm{n}=158,13.1 \%$ ), treatment effect size (Cohen's d) was 0.85 (mean change -27.6 in the lurasidone group versus -12.1 in the placebo group). Treatment effects sizes were 0.34 in patients who had 1 prior hospitalization $(\mathrm{n}=115,9.6 \%), 0.46$ in patients who had 2 prior hospitalizations ( $\mathrm{n}=145,12.1 \%$ ), 0.38 in patients who had 3 prior hospitalizations $(\mathrm{n}=138,11.5 \%), 0.34$ in patients who had 4 or more prior hospitalizations $(\mathrm{n}=646,53.7 \%)$. Statistical interaction test showed that the number of prior hospitalizations was a moderating variable for treatment effect size, with significant difference between treatment effect and prior hospitalizations ( 0 versus 1 or more) $(\mathrm{p}<0.01)$.

CONCLUSIONS: In this pooled lurasidone database analysis, patients with a history of prior hospitalization (and presumed relapse) in schizophrenia had diminished treatment effect size compared to those with no prior hospitalization. Trajectories of improvement (by number of past hospitalizations) indicated that a slower rate of improvement (more gradual efficacy slope) occurred as the number of prior hospitalizations increased. Number of past hospitalizations seems to moderate treatment responsiveness in patients with schizophrenia. These findings suggest that psychotic relapse may impair the ability to achieve optimal response in patients with schizophrenia.

FUNDING: This study was funded by Sunovion Pharmaceuticals Inc.

\section{Effect of Lurasidone on Prosocial Functioning in Patients with Schizophrenia: A Pooled Analysis of Five Short-Term, Placebo-Controlled Studies}

Andrei Pikalov, MD, PhD'; Michael Tocco, PhD'; Hanzhe Zheng, PhD'; Josephine Cucchiaro, $P h D^{\prime}$ and Antony Loebel, $\mathrm{MD}^{\prime}$

${ }^{1}$ Sunovion Pharmaceuticals Inc.

STUDY OBJECTIVE: To assess the efficacy of lurasidone for improving prosocial behavior in patients with schizophrenia.

METHODS: Patient-level data were pooled from 5 randomized, double-blind, placebo-controlled, 6-week studies of fixed-dose lurasidone $(40-160 \mathrm{mg} / \mathrm{d})$ in the treatment of patients with an acute exacerbation of schizophrenia. This analysis included patients who had data at baseline and at least 1 post-baseline assessment for the Positive and Negative Syndrome Scale (PANSS). The prosocial factor score was calculated as the sum of 6 PANSS item scores: active social avoidance, emotional withdrawal, passive social withdrawal, stereotyped thinking, hallucinatory behavior, and suspiciousness. Lower scores indicate better prosocial behavior. Mixed-model repeated-measures analysis was used to compare change from baseline in the prosocial factor score for patients receiving lurasidone versus placebo.

RESULTS: This analysis included 1526 patients (lurasidone, $\mathrm{n}=1030$; placebo, $\mathrm{n}=496$ ). Mean baseline PANSS prosocial factor scores were 23.0 and 23.2 in the lurasidone and placebo groups, respectively. Leastsquares (LS) mean change from baseline to Week 6 study endpoint was -6.3 for lurasidone (dose groups pooled) and -3.7 for placebo $(\mathrm{P}<0.001$; effect size $=0.57)$. Lurasidone (dose groups pooled) provided significantly greater improvement than placebo in the prosocial factor score beginning as early as Day $3 / 4$ and continuing through the 6 -week study period $(\mathrm{P} \leq 0.001)$. When each dose of lurasidone was analyzed separately, LS mean change from baseline to Week 6 endpoint in the prosocial factor score was -5.9 for $40 \mathrm{mg} / \mathrm{d},-6.3$ for 80 
$\mathrm{mg} / \mathrm{d}$, -6.1 for $120 \mathrm{mg} / \mathrm{d}$, and -7.3 for $160 \mathrm{mg} / \mathrm{d}$. All doses of lurasidone were superior to placebo from Week $1(\mathrm{P} \leq 0.001)$ through Week $6(\mathrm{P}<0.001$; effect sizes ranged from 0.49 for $40 \mathrm{mg} / \mathrm{d}$ to 0.75 for $160 \mathrm{mg} / \mathrm{d}$ ). In these studies, the most common adverse events $(\geq 10 \%$ of patients receiving lurasidone and incidence $>$ placebo) associated with lurasidone versus placebo were akathisia ( $16.1 \%$ vs $4.6 \%)$, somnolence $(12.6 \%$ vs $6.2 \%)$, and insomnia ( $10.5 \%$ vs $8.9 \%)$.

CONCLUSIONS: In this pooled analysis of short-term studies of patients with schizophrenia, lurasidone 40 $\mathrm{mg} / \mathrm{d}$ to $160 \mathrm{mg} / \mathrm{d}$ produced significantly greater improvement than placebo in prosocial behavior. Effect sizes indicate that these were clinically meaningful differences, suggesting that lurasidone may improve prosocial functioning in patients with schizophrenia.

FUNDING: This study was sponsored by Sunovion Pharmaceuticals Inc.

ClinicalTrials.gov identifiers: NCT00088634, NCT00549718, NCT00615433, and NCT00790192. One study was completed prior to the requirement to register trials.

\section{REFERENCES:}

Pikalov A, Tocco M, Zheng H, Cucchiaro J, Loebel A. Effect of lurasidone on prosocial functioning in patients with schizophrenia: a pooled analysis of five short-term, placebo-controlled studies. Presented at the American Society of Clinical Psychopharmacology Annual Meeting; June 22-25, 2015; Miami, FL.

\section{Randomized Pilot Study of Two Maintenance Strategies of Neurostar Transcranial Magnetic Stimulation in Patients with Major Depression}

\author{
Noah S. Philip, $M D^{\prime}$ and David G Brock, $M D^{2}$ \\ ${ }^{1}$ Butler Hospital, Providence, RI \\ ${ }^{2}$ Neuronetics, Inc, Malvern, PA
}

ABSTRACT: Study Objective: Transcranial magnetic stimulation (TMS) is an effective acute treatment for patients with major depressive disorder (MDD), but an important unanswered question is what is the ideal strategy for maintaining symptom benefit once a patient has had an initial response to TMS. This pilot study examined the efficacy over one-year of once monthly scheduled maintenance TMS treatment compared to monthly observation for the prevention of symptomatic worsening in patients who had shown a clinical response to acute TMS treatment.

METHODS: Pharmacotherapy-free patients with unipolar, non-psychotic MDD, who had failed to receive benefit from prior antidepressant treatment were enrolled. Patients meeting criteria (HAMD17 <15 and >25\% improvement) at the end of a fixed six-week acute treatment were randomized to either once monthly maintenance TMS or monthly observation. Patients in either arm received TMS reintroduction for protocol defined symptomatic worsening (HAMD17 $\geq 16$ and worsening $\geq 25 \%$ ).

RESULTS: Sixty-seven medication-free patients were enrolled of whom 49 met randomization criteria $(\mathrm{N}=23$ to scheduled treatment and $\mathrm{N}=26$ to observation only); 42 of $67(63 \%)$ patients remitted with acute treatment (HAMD17 <8). The primary outcome, comparison of evaluable patients not requiring TMS reintroduction at any time point during the maintenance phase, was 5/16 patients $(31.3 \%)$ with maintenance vs. $4 / 16$ patients $(25.0 \%)$ for the observation $\operatorname{arm}(\mathrm{p}=\mathrm{NS})$. The mean (SD) number of days after end of acute TMS series until the first re-introduction TMS treatment was 103.5 (61.3) days for monthly TMS vs. 97.8 (59.1) days for observation only $(\mathrm{p}=\mathrm{NS})$. Of those participants needing retreatment during the maintenance phase, $17(58.6 \%)$ required 1 retreatment period, and $10(34.4 \%)$ required 2 .

CONCLUSIONS: In this pilot study, prophylactic TMS delivered once per month did not significantly delay the time until re-treatment was needed or reduce the proportion of patients who needed re-treatment. The study did confirm that it is feasible to maintain treatment resistant MDD patients with TMS alone over the course of 1 year. Although TMS reintroduction was needed in the majority of this medication-free group, most patients required no more than two unique retreatment series. The results suggest that a brain stimulation treatment may require different, sequenced approaches to maintenance, for example, observation at first, moving to prophylaxis as a second stage and reserving combination with pharmaceuticals as a last step.

FUNDING: This study was funded by Neuronetics, Inc.

Clinical trial posted on www.clinicaltrials.gov, NCT 01415154

\section{Psychosis or Ptsd: My 12 Year Old Son Thinks he is Toxic}

Sultana Jahan, $M D$

University of Missouri, Columbia, MO

STUDY OBJECTIVE: To describe the differential diagnosis and clinical course of a child who presented with symptoms of possible psychosis, with a history of repeated trauma. 
METHOD: 12 year old B presented to our university child and adolescent psychiatry outpatient clinic for an evaluation of strange behavior and unusual thinking. According to his mother, he had moved to the United States several months previously. Since coming to the United States, he had been catching invisible objects from the air and putting them in his mouth. When she asked him about it, he answered, "I am catching the toxins and swallowing them so that no one gets sick". He also had strange vocalizations without any explanation. He thought that he was toxic. Furthermore, he was suspicious that a man had been following him.

While talking to the patient individually he reported that he was sexually molested by his classmates in his native country. He became terrified whenever he saw students with similar ethnic background at his new school in the USA. He described having nightmares and flashbacks related to the molestation. His sleep was interrupted due to extreme anxiety \& nightmares.

Past psychiatric history, Medical and surgical history was insignificant. Substance abuse history was negative.

His developmental history was normal. His family history was notable for his mother having suffered from OCD as a teenager.

His mental status exam was positive for extremely anxious mood and affect. He was hyper-vigilant. $\mathrm{He}$ presented unusual behavior; he was catching toxins from the air and swallowing them. He was also paranoid.

RESULTS: After the initial evaluation, he was given a presumptive diagnosis of post traumatic stress disorder (PTSD). He was started on low doses of sertraline and risperidone. He also received trauma focused cognitive behavioral therapy. Gradually the doses of risperidone and sertraline were increased to $1.5 \mathrm{mg}$ orally bid and $150 \mathrm{mg}$ orally daily respectively. A year later paranoia, strange behavior, and unusual thinking subsided and then his PTSD symptoms subsided. Another year later his risperidone was slowly tapered off. Psychotic symptoms did not return once risperidone was stopped. His PTSD symptoms were well managed with sertraline alone. Eventually the patient graduated high school and is currently a junior at his local university.

CONCLUSIONS: Patients with PTSD may also present with the symptoms of psychosis. It took more than 2 years to verify that patient's psychotic symptoms were manifestation of PTSD. The key point is to be cautious before making diagnoses of schizophrenia when patients present with symptoms of psychosis and PTSD.

\section{REFERENCE:}

Sareen J, Cox BJ, Goodwin RD. Asmundson GJG. Co-occurrence of posttraumatic stress disorder with positive psychotic symptoms in a nationally representative sample. Journal of Traumatic Stress. 2005; 18: $313-322$.
An Evidence-Based Analysis of The Relationship Between Lithium Dosage And Related Safety and Efficacy Outcomes in Adults with Bipolar Disorder

Doug Taylor, $B S^{\prime}$; Nicole Coolbaugh, BA'; Susan Martin, PhD' and Philip Sjostedt, BPharm'

${ }^{1}$ Institute for Evidence Based Medicine, New Hope, PA

STUDY OBJECTIVES: To conduct an analysis of efficacy and safety of lithium in adult patients with bipolar disorder (BPD) to determine if higher doses of lithium are clinically appropriate. This analysis also seeks to provide evidence-based guidance for lithium use in adult BPD patients based on the included results.

METHODS: Evidence-based treatment guidelines and clinical trials for bipolar disorder published in the last 5 years were reviewed. The guidelines and clinical trial results were compared with the lithium carbonate (controlled- and immediate-release) prescribing information to determine the relationship between efficacy, safety, and dosage in adult patients with bipolar disorder. The safety analysis included prevalence of adverse events and study discontinuation rates. Efficacy was defined as using the remission criteria employed by the authors of the original trials.

RESULTS: A total of 5 guidelines and 43 clinical trials were analyzed. Adverse events were positively correlated with higher doses of lithium across all reviewed clinical trials, with no significant corresponding increase in efficacy at the higher doses. Treatment compliance, as measured by study completion rates, was lower in patients receiving higher doses of lithium in both monotherapy and adjunctive therapy trials. Furthermore, switching from high- to low-dose lithium results in decreases in psychiatric morbidity and side effects. For patients with higher serum lithium concentrations, discontinuation rates for reasons other than acute mood episodes were $36.0 \%$, compared to $23.1 \%$ in patients receiving a lower dose.

CONCLUSIONS: Recent clinical data and evidence-based guidelines show no statistically significant correlation between increased serum lithium concentrations and remission of bipolar symptoms. Higher lithium concentrations are also associated with an increase in adverse events. Previous suggestions that patients must balance the efficacy improvements associated with high lithium doses with increased side effect prevalence require reevaluation.

FUNDING: This study was funded by Institute for Evidence Based Medicine. 
Effect of Online Continuing Medical Education in Bipolar Depression: A Path to Improved Care?

Jovana Lubarda, PhD'; Piyali Chatterje ${ }^{\prime}$; Margaret McLaughlin, PhD' and Roger S. McIntyre, $M D^{2,3}$

${ }^{1}$ Medscape LLC, New York, NY

${ }^{2}$ Head, Mood Disorders Psychopharmacology Unit, University Health Network, University of Toronto,

Toronto, Ontario, Canada

${ }^{3}$ Professor of Psychiatry and Pharmacology, University of Toronto, Toronto, Ontario, Canada

OBJECTIVES: To evaluate effects of multiple online continuing medical education (CME) activities on competence and performance of physicians managing bipolar I (BP-I) depression.

METHODS: Psychiatrists and primary care physicians (PCPs) participated in 3 online CME formats to address key concepts in BP-I depression: a case-based expert interview, a video panel discussion, and an in-depth textbased monograph. Effect of each activity was assessed through case-based survey questions pre-, post-, and again 30-60 days on follow-up on linked participants who served as their own controls. McNemar's chi-squared tests were used to determine statistical significance $(\mathrm{P}<0.05)$; educational effect size of each activity was calculated using Cramer's V, based on pre- and postCME performance. Individual questions from each CME activity were then grouped into critical themes in BP-I management, including diagnosis, treatment, and ongoing long-term management, and physician responses were averaged to provide a comprehensive view of educational effects. The CME activities launched online between $12 / 18 / 13$ to $9 / 17 / 2014$ and data were collected for 337 days.

RESULTS: Both psychiatrists (average $\mathrm{V}=0.42$, moderate effect) and PCPs (average $\mathrm{V}=0.32$, robust effect) improved competence and performance following CME activities. On the theme of diagnosis of BP-I depression, on average, $67 \%$ of psychiatrists answered correctly on pre-assessment, increasing to $77 \%$ post-assessment ( $\mathrm{n}=411 ; \mathrm{P}<.001$ ), and $73 \%$ of PCPs answered correctly on pre-assessment increasing to $85 \%$ on post-assessment ( $\mathrm{n}=324 ; \mathrm{P}<.001)$. On follow-up on a smaller sample ( $\mathrm{n}=55$ psychiatrists; $\mathrm{n}=28 \mathrm{PCPs}$ ), all the responses remained close to post-assessment levels, indicating strong retention.

On themes of individualized treatment and selection of first-line treatments, on average, $48 \%$ of psychiatrists answered correctly on pre-assessment, rising to $67 \%$ post-assessment $(\mathrm{n}=3683 ; \mathrm{P}<0.05)$, and $28 \%$ of PCPs answered correctly on pre-assessment, rising to $64 \%$ post-assessment $(\mathrm{n}=1301 ; \mathrm{P}<0.05)$. On follow-up on a smaller sample, psychiatrists $(\mathrm{n}=267)$ improved performance by $7.5 \%$ on average, while PCPs $(n=64)$ declined by $13 \%$ on average.

On the theme of long-term management of BP-I depression, on average, $28 \%$ of psychiatrists answered correctly on pre-assessment, rising to $68 \%$ postassessment $(\mathrm{n}=3683 ; \mathrm{P}<0.05)$, and $38 \%$ of PCPs answered correctly on pre-assessment, rising to $60 \%$ post-assessment $(\mathrm{n}=643 ; \mathrm{P}<0.05)$. On follow-up on a smaller sample, psychiatrists $(\mathrm{n}=447)$ retained performance completely, while PCPs $(n=64)$ declined by $\mathbf{8 \%}$ on average.

CONCLUSIONS: Online CME in various formats, including expert interview, panel discussion, and text-based monograph, improved evidence-based decisions of psychiatrists and PCPs in BP-I depression, which may translate into clinical practice and patient care.

FUNDING: This study was funded by an independent educational grant from Sunovion Pharmaceuticals Inc.

\section{Continuing Medical Education Improves Knowledge on Latest Clinical Data in Schizophrenia and Bipolar Disorder}

\author{
Jovana Lubarda, PhD'; Piyali Chatterje' and \\ Roger S. McIntyre, $M D^{2,3}$ \\ ${ }^{1}$ Medscape LLC, New York, NY \\ ${ }^{2}$ Head, Mood Disorders Psychopharmacology Unit, \\ University Health Network, University of Toronto, \\ Toronto, Ontario, Canada \\ ${ }^{3}$ Professor of Psychiatry and Pharmacology, University \\ of Toronto, Toronto, Ontario, Canada
}

STUDY OBJECTIVES: This study assessed whether online continuing medical education (CME) can improve clinical knowledge of psychiatrists and primary care physicians (PCPs) regarding the latest data on current and emerging antipsychotic therapies for managing schizophrenia (SCZ) and bipolar disorder (BP).

METHODS: The effectiveness of an online video-based panel discussion focusing on summaries and expert perspectives on emerging clinical data on antipsychotics for SCZ and BP in 2014 was analyzed. The educational effect of the program was assessed by comparing the same group of participants' responses to 4 identical preand post-CME assessment questions. A paired 2-tailed t-test was used to assess whether the mean postassessment score was different from the mean preassessment score. McNemar's chi-squared statistic was used to determine statistical significance and the effect of education was calculated using Cohen's d which compared the mean pre-assessment versus postassessment scores. 
RESULTS: Responses from 631 psychiatrists and 135 PCPs who answered all pre/post questions were analyzed. Participation in online CME discussing emerging clinical data in SCZ and BP demonstrated the following: For psychiatrists who participated in the CME activity, correct responses on post-assessment questions were between $54 \%$ and $76 \%$ higher after CME completion versus pre-assessment, with an overall large educational effect size of $d=1.231(\mathrm{P}<.05)$. While only $67(11 \%)$ of psychiatrists answered all 4 questions correctly on preassessment, 338 (54\%) answered all 4 questions correctly on the post-assessment.

For PCPs who participated in the CME activity, correct responses on post-assessment questions were between $55 \%$ and $76 \%$ higher after CME completion versus pre-assessment, with an overall large educational effect size of $\mathrm{d}=1.244(\mathrm{P}<.05)$. While only $18(13 \%)$ of PCPs answered all 4 pre-assessment questions correctly, $72(53 \%)$ answered all questions correctly on the post-assessment.

Significant improvements for both psychiatrists and PCPs were noted regarding correctly identifying relevant attributes of antipsychotic medications and understanding the results of clinical trial data, such as for cariprazine and ITI-007.

CONCLUSIONS: Online CME utilizing a video-based panel discussion to communicate the latest clinical data in SCZ and BP was effective in improving physician knowledge, which may facilitate adoption in clinical practice.

FUNDING: This study was funded by independent educational grant from Forest Laboratories, Inc.

\section{A Case of 31-Year-0ld Male with Chronic History of Toluene use Causing Parkinsonism}

Manoj Puthiyathu, $\mathrm{MD}^{\prime}$; Shahan Sibtain, $M D^{\prime}$ and Rumana Rahmani, $M D^{\prime}$

${ }^{1}$ Bergen Regional Medical Center, Paramus, NJ

STUDY OBJECTIVES: a) Diagnostic criteria for Parkinsonism and differentiating it from Parkinson Disease b) Treatment modalities for case of toluene induced Parkinsonism.

ABSTRACT: Parkinsonism is a syndrome defined by a specific set of clinical findings which overlaps Parkinson's disease. Many etiological factors are linked to Parkinsonism which ranges from Parkinson's disease, being most common, to many medications, toxins and chemical compounds. Toluene is one such chemical compound which is a colorless liquid used in industrial solvents. Due to its intoxicating properties it is also used as an inhalant by many, even though it has been established that it can cause severe neurological damage. We report a case of a 31-year-old Caucasian male presented for addiction treatment with prior multiple hospitalizations for poly-substance abuse and positive history of chronic use of toluene. He used toluene for over a period of 2 years several times a week. He was exhibiting anxiety, labile mood and tremors which got worse over the years and led him to seek treatment. Patient reported that even after medication there was no improvement in his tremors and it was more of postural type and were more profound in upper extremities. On further evaluation patient showed no other symptoms which could conclude the diagnosis of essential tremors or Parkinson's disease. The use of toluene and no improvement in tremors after medication were more in favor of Parkinsonism. Research studies have established a strong association between the chronic use of toluene with Parkinsonism. Our case highlights these associations and diagnostic criteria for Parkinsonism and further supports the conclusions of these research studies.

\section{Work Readiness After Treatment with Aripiprazole Once-Monthly and Paliperidone Palmitate: Results from Qualify, A Head-to- Head Study in Schizophrenia}

Steven G. Potkin, $M D^{\prime}$; Jean-Yves Loze, MD, $\mathrm{MSc}^{2}$ Carlos Forray, $\mathrm{MD}^{3}$; Ross A. Baker, PhD, MBA Christophe Sapin, $\mathrm{MSc}^{5}$; Timothy Peters-Strickland, $\mathrm{MD}^{4}$; Maud Beillat, MSc$c^{5}$; Jessie Chambers, PhD ${ }^{4}$; Phyllis Salzman, PhD ; Anna-Greta Nylander, PhD, $\mathrm{MBA}^{6}$; Peter Hertel, PhD ${ }^{6}$; Henrik Steen Andersen, $\mathrm{MSc}^{6}$; Anna Eramo, $\mathrm{MD}^{7}$; Karina Hansen, $\mathrm{PhD}, \mathrm{MSc}^{5}$ and Dieter Naber, $M D^{8}$

${ }^{1}$ Department of Psychiatry and Human Behavior, University of California, Irvine, CA

${ }^{2}$ Otsuka Pharmaceutical Europe, Wexham, UK

${ }^{3}$ Lundbeck Research, Paramus, NJ

${ }^{4}$ Otsuka Pharmaceutical Development \& Commercialization, Inc., Princeton, NJ

${ }^{5}$ Lundbeck SAS, Issy-les-Moulineaux, France

${ }^{6}$ H. Lundbeck A/S, Valby, Denmark

${ }^{7}$ Lundbeck LLC, Deerfield, IL

${ }^{8}$ University Medical Center Hamburg-Eppendorf, Hamburg, Germany

OBJECTIVES: This study compared the effectiveness of aripiprazole once-monthly $400 \mathrm{mg}$ (AOM 400) and paliperidone palmitate once-monthly (PP) on symptoms and functioning in patients with schizophrenia. Secondary effectiveness analyses of AOM 400 and PP on the Readiness for Work Questionnaire (WoRQ), a scale measuring functional capacity, are reported here. 
METHODS: QUALIFY was a 28-week, randomized, open-label, head-to-head study (NCT01795547) of 2 atypical long-acting injectable antipsychotics, AOM 400 and PP (flexible dosing 78-234 mg/month as paliperidone palmitate) in patients with schizophrenia aged 18-60 years. Patients were randomized to 3 weeks' conversion to oral aripiprazole or paliperidone followed by 5 weeks' initiation of AOM 400 or PP treatment and continued injections every 4 weeks for 20 weeks. Mean ą SD dose at week 24 was 387 ą34 mg AOM and 172 ą52 $\mathrm{mg}$ PP. The primary endpoint was change from baseline to week 28 in the Heinrichs-Carpenter Quality-of-Life Scale (QLS) total score, analyzed with a mixed model for repeated measures (MMRM). The Tolerability and Quality of Life Questionnaire (TooL) is a patient-rated assessment of the impact of specific antipsychotic medication side effects on patients' quality of life; total scores range from 8 (no impact) to 32 (maximum impact). Change from baseline to week 28 on TooL total score (MMRM) was a predefined secondary endpoint. As an additional predefined endpoint, change from baseline in clinician-rated WoRQ total scores was assessed with analysis of covariance. Logistic regression was used to assess readiness for work at week 28. Lower WoRQ total scores indicate better clinical condition.

RESULTS: In the primary analysis, AOM $400(\mathrm{n}=136)$ showed superiority vs $\mathrm{PP}(\mathrm{n}=132)$ on improvement in QLS total score (least squares mean [LSM] treatment difference: $4.67 ; 95 \%$ CI: $[0.32,9.02], \mathrm{P}=0.036$ ). The change in TooL total scores showed a nonsignificant trend in favor of AOM 400 (LSM treatment difference: $-0.70 ; 95 \%$ CI: $[-1.51,0.12], \mathrm{P}=0.095)$. At baseline, the clinical assessment of readiness to work (yes/no) was similar between groups. At week 28, 29/110 (26.4\%) of patients on AOM 400 had changed from "no" to "yes" in readiness to work compared to $12 / 98(12.2 \%)$ of patients on $\mathrm{PP}$, and the odds of being rated as ready for work were higher for AOM 400 vs PP (adjusted odds ratio: 2.67; 95\% CI:[1.39, 5.14], $\mathrm{P}=0.003)$. Significant improvements were also found in change in WoRQ total scores with AOM 400 over PP (LSM treatment difference: -1.16 ; 95\% CI: [-1.96, -0.37], $\mathrm{P}=0.004)$.

CONCLUSION: Superior improvements in health-related quality of life and functioning measured with QLS total score were found for AOM 400 vs PP treatment in patients with schizophrenia. The primary results were supported by significant improvements with AOM 400 treatment relative to $\mathrm{PP}$ on the clinician-rated WoRQ, suggesting that patients' functional capacity increased after AOM 400 treatment.

FUNDING: This study was funded by Otsuka Pharmaceutical Development \& Commercialization, Inc.

\section{Treatment Modalities for A Case of Olfactory Reference Syndrome Causing Substance Abuse}

Manoj Puthiyathu, $M D^{\prime}$; Shahan Sibtain, $M D^{\prime}$ and
Rumana Rahmani, $M D^{\prime}$

${ }^{1}$ Bergen Regional Medical Center, Paramus, NJ

ABSTRACT: Olfactory reference syndrome (ORS) is a delusional disorder having a false belief about abnormal odor. This odor can range from body odor to any material and nonhuman odor which is not perceived by anyone in reality. The pathophysiology of ORS is not completely understood but symptoms overlap many psychiatric illnesses thus imposing a challenge for doctors to diagnose the disease. We present a case of a 26-year-old male presented for addiction treatment and symptoms showing some features of depression and delusion. On detailed examination and evaluation the patient complain that he has a bad body odor and he takes GHB because it helps him forget about his body odor. $\mathrm{He}$ showed symptoms of depression and avoided going to parties and social gatherings as he thinks others can smell his odor and react by making gestures, which according to patient was in reaction to his body odor. He reported anxiety and restlessness when attending social gatherings or even in crowded places but the underlying reason was his concern that his body odor may be perceived by others. On physical examination there was no foul body odor or any other smell perceived that could be the cause of concern in patient. No other physical abnormality was present. Patient did show depressive symptoms but no symptoms of panic disorder, social phobia or obsessive compulsive disorder. Our case highlights the diagnostic criteria for olfactory reference syndrome, how to differentiate it from other psychiatric disorders with similar spectrum of symptoms, and also explains treatment modalities that support the previous conclusions of the research done on ORS.

\section{Qualify: A Randomized Head-To-Head study Of Aripiprazole Once-Monthly and Paliperidone Palmitate in the Treatment of Schizophrenia}

Steven G. Potkin, $M D^{\prime}$; Jean-Yves Loze, MD, $M S c^{2}$; Carlos Forray, $\mathrm{MD}^{3}$; Ross A. Baker, PhD, $\mathrm{MBA}^{4}$; Christophe Sapin, $\mathrm{MSc}^{5}$; Timothy Peters-Strickland, $\mathrm{MD}^{4}$; Kamila Piekos, PharmD ${ }^{4}$; Maud Beillat, $\mathrm{MSc}^{5}$; Anna-Greta Nylander, PhD, $M B A^{6}$; Peter Hertel, $P h D^{6}$; Henrik Steen Andersen, $\mathrm{MSc}^{6}$; Anna Eramo, $\mathrm{MD}^{7}$; Karina Hansen, $\mathrm{PhD}, \mathrm{MSc}^{5}$ and Dieter Naber, $M D^{8}$

${ }^{1}$ Department of Psychiatry and Human Behavior, University of California, Irvine, CA

${ }^{2}$ Otsuka Pharmaceutical Europe, Wexham, UK 
${ }^{3}$ Lundbeck Research, Paramus, NJ

${ }^{4}$ Otsuka Pharmaceutical Development \&

Commercialization, Inc., Princeton, NJ

${ }^{5}$ Lundbeck SAS, Issy-les-Moulineaux, France

${ }^{6}$ H. Lundbeck A/S, Valby, Denmark

${ }^{7}$ Lundbeck LLC, Deerfield, IL

${ }^{8}$ University Medical Center Hamburg-Eppendorf, Hamburg, Germany

OBJECTIVE: The QUALIFY study directly compared treatment effectiveness of aripiprazole once-monthly $400 \mathrm{mg}$ (AOM 400) and paliperidone palmitate oncemonthly (PP) using the Heinrichs-Carpenter Quality-ofLife Scale (QLS), a validated measure of effectiveness designed to assess intrapsychic, interpersonal, and instrumental functioning, as the primary endpoint in patients with schizophrenia.

METHODS: QUALIFY was a 28-week, randomized, openlabel, rater-blinded, head-to-head study (NCT01795547) of 2 atypical long-acting injectable anti-psychotics (LAIs), AOM 400 or PP (flexible dosing 78-234 mg/ month as paliperidone palmitate), to evaluate effectiveness in patients with schizophrenia. Included patients were aged 18-60 years, needing a change from current oral antipsychotic treatment and, in the investigator's judgment, would benefit from LAI treatment. The study comprised 3-week conversion to oral aripiprazole or oral paliperidone, initiation of AOM 400 or PP according to their labels, and continued with injections every 4 weeks for 20 weeks. The study was designed to test noninferiority, with the pre-defined possibility for subsequent superiority testing if the noninferiority criterion was met. A change in QLS total score $>=5.3$ points is considered clinically relevant, and the noninferiority margin was -5 points on the QLS total score. The primary endpoint was change from baseline to week 28 in raterblinded QLS total score and was analyzed with a mixed model for repeated measures.

RESULTS: Of 295 randomized patients, 67.6\% (100/148) in the AOM group and $56.5 \%(83 / 147)$ in the PP group completed 28 weeks of treatment. In treated patients, adverse events (AEs) were the most frequent reason for discontinuation (AOM: 11.1\% [16/144]; PP: 19.7\% [27/137]). At week 24, mean \pm SD dose was $387 \pm 34 \mathrm{mg}$ $\mathrm{AOM}$ and $172 \pm 52 \mathrm{mg} \mathrm{PP}$ as paliperidone palmitate. The between-group least squares mean (LSM) difference in change from baseline to week 28 in QLS total score was 4.67 (95\% CI: $0.32-9.02, \mathrm{P}=0.036$ ), confirming noninferiority and establishing superiority of AOM 400 vs PP. The respective LSM changes were $7.47 \pm 1.53$ for AOM 400 (baseline $66.0 \pm 21.7$ ) and $2.80 \pm 1.62$ for PP (baseline $62.9 \pm 21.5$ ). Both treatments were generally well tolerated; AEs occurring in the treatment continuation phase at rates $>=5 \%$ in either group were weight increased (AOM 400: 12/119 [10.1\%]; PP: 17/ 109 [15.6\%]), psychotic disorder (AOM 400: 3/ 119 [2.5\%]; PP: 6/109 [5.5\%]), and insomnia (AOM 400: 3/119 [2.5\%]; PP: 6/109 [5.5\%]).

CONCLUSIONS: Superior improvements on health-related quality of life and functioning as measured by the clinician-rated QLS and numerically lower rates of allcause discontinuation suggest greater overall effectiveness for AOM 400 vs PP. The observed changes from baseline in QLS total score after AOM 400 treatment are considered to represent a clinically relevant improvement in functioning, in contrast to PP.

FUNDING: This study was funded by

\section{Tetrabenazine Induced Rapid Improvement of Tardive Dyskinesia: A Case Report}

\author{
Asif Khan, $M D^{\prime}$ and David Leavitt, $M D^{\prime}$ \\ ${ }^{1}$ Department of Psychiatry, St. Mary Mercy Hospital, \\ Livonia, MI
}

INTRODUCTION: Tardive dyskinesia (TD) is a hyperkinetic movement disorder that appears with a delayed onset after prolonged use of dopamine receptor blocking agents. There is no generally accepted treatment for TD following neuroleptic use. Several proposed therapeutic strategies include switching of high potency to lower potency neuroleptics, discontinuation of neuroleptics, use of GABAergic agents especially clonazepam, use of antioxidant vitamin $\mathrm{E}$, amantadine and tetrabenazine. Tetrabenazine functions as a reversible depletor of monoamines from nerve terminals.

CASE REPORT: We report a case of a 67 -year-old female with history of mood disorder, who developed severe lower limb TD after exposure to atypical antipsychotics for a brief period of 3-4 months. Her TD symptoms did not respond to switching and discontinuation of the neuroleptics, clonazepam, propranolol and vitamin E. Her concurrent depression was successfully treated in the meantime. After more than a year with continued symptoms of unchanged intensity, the patient in collaboration with the movement disorder center of the University of Michigan was treated with tetrabenazine monotherapy, with rapid improvement of the severity of the symptoms in about 4 weeks.

DISCUSSION: This case illustrates the potential of management of severe TD with tetrabenazine. Tetrabenazine is FDA approved for treatment of chorea and can be utilized as treatment option in tardive dyskinesia, with careful monitoring of the side effects including severe depression. Further longitudinal studies will help assess the efficacy of this drug as monotherapy in the treatment of tardive dyskinesia. 
Efficacy and Safety of Vortioxetine in Adults with Major Depressive Disorder and Comorbid Diabetes Mellitus: Pooled Analyses of Short- and Long-Term Studies

Atul R. Mahableshwarkar, $M D^{\prime}$; Dapo Tomori, $M D^{\prime}$; Wei Zhong, PhD'; William Palo, $M S^{\prime}$ and Louis Mini, $M^{\prime}$

1 Takeda Development Center Americas, Deerfield, IL

OBJECTIVE: The association between major depressive disorder (MDD) and diabetes mellitus (DM) is of increasing clinical interest. This post hoc analysis evaluated short-term (ST) and long-term (LT) safety and efficacy of vortioxetine $5-20 \mathrm{mg} / \mathrm{d}$ in adults with MDD and comorbid DM.

METHOD: Data were pooled from 12 ST (6-8wks), randomized, placebo (PBO)-controlled studies in which vortioxetine $5-20 \mathrm{mg} / \mathrm{d}$ was investigated in adults with MDD. Data from 5 LT, open-label (OL) extension studies $(\leq 52 \mathrm{wks})$ of vortioxetine $5-20 \mathrm{mg} / \mathrm{d}$ were analyzed separately. The efficacy outcome measure was the change in total score on the Montgomery-Åsberg Depression Rating Scale (MADRS) from baseline to study endpoint. Safety and tolerability assessment was based on the nature, incidence, and severity of adverse events (AEs) and clinical laboratory findings during treatment.

RESULTS: Out of 5222 patients in the 12 ST studies, 166 $(3.2 \%)$ had comorbid DM (PBO $\mathrm{n}=64$; vortioxetine $\mathrm{n}=102$ ) and were included in the safety analysis. The full analysis set included 61 PBO and 101 vortioxetine patients. Depression severity was similar for PBO and vortioxetine (baseline MADRS: 31.9, 32.0, respectively). Vortioxetine produced significant improvement in clinical efficacy vs PBO after 6-8wks (MADRS $\Delta-3.5$, $\mathrm{P}=0.025$; ANCOVA, LS means). Patients continuing on OL vortioxetine for $\leq 52 \mathrm{wks}$ ( $\mathrm{n}=68$, including those who switched to vortioxetine after the initial treatment period) demonstrated ongoing benefit (MADRS $\Delta-8.8$ vs OL baseline). In MDD patients with DM during ST treatment, the incidence of AEs for PBO and vortioxetine was $57.8 \%$ and $62.7 \%$, respectively; treatment-related AEs (TRAEs) was $48.4 \%$ and $46.1 \%$; AEs leading to treatment withdrawal was $1.6 \%$ and $8.8 \%$; and serious AEs (SAEs) was $1.6 \%$ and $2.9 \%$. Nausea $(9.4 \%, 20.6 \%)$, headache $(15.6 \%, 12.7 \%)$, and vomiting $(0 \%, 6.9 \%)$ were the most common AEs (incidence $\geq 5 \%$ ). During LT vortioxetine treatment the incidence of AEs in MDD patients with DM (76.5\%) was similar to that of the total population, as was the incidence of TRAEs (57.4\%), AEs leading to withdrawal (7.4\%), and SAEs (5.9\%). The most common AEs during LT treatment were nausea (26.5\%), headache $(11.8 \%)$, dry mouth $(8.8 \%)$, nasopharyngitis (8.8\%), abdominal pain upper $(5.9 \%)$, diarrhea (5.9\%), fatigue $(5.9 \%)$, and vomiting $(5.9 \%)$. No significant changes from baseline or differences vs PBO in weight or key laboratory parameters, including blood glucose, were noted after ST or LT treatment with vortioxetine.

CONCLUSIONS: In all 12 ST studies, vortioxetine $5-20 \mathrm{mg} / \mathrm{d}$ was efficacious vs PBO in reducing MADRS score in MDD patients with DM. MADRS scores continued to improve during $52 \mathrm{wks}$ of OL treatment. No unexpected AEs, changes in laboratory parameters or safety concerns were apparent in this small sample relative to the total population. Based on these data, vortioxetine is a consideration for the treatment of MDD in patients with comorbid DM.

FUNDING: This study was funded by Takeda Pharmaceutical Company, Ltd and H. Lundbeck A/S.

\section{Clinical Global Impression After Aripiprazole Once-Monthly and Paliperidone Palmitate: Results from Qualify, A Head-To-Head Study in Schizophrenia}

Dieter Naber, $\mathrm{MD}^{\prime}$; Karina Hansen, PhD, $\mathrm{MSc}^{2}$; Carlos Forray, $\mathrm{MD}^{3}$; Ross A. Baker, PhD, MBA ${ }^{4}$; Christophe Sapin, $\mathrm{MSc}^{2}$; Maud Beillat, $\mathrm{MSc}^{2}$; Timothy PetersStrickland, $M D^{4}$; Anna-Greta Nylander, PhD, $M B A^{5}$; Peter Hertel, PhD ${ }^{5}$; Henrik Steen Andersen, MSc ${ }^{5}$; Rolando Veloso, PharmD $D^{6}$ Anna Eramo, $M D^{6}$; JeanYves Loze, MD, MSc ${ }^{7}$ and Steven G. Potkin, $M D^{8}$

${ }^{1}$ University Medical Center Hamburg-Eppendorf, Hamburg, Germany

${ }^{2}$ Lundbeck SAS, Issy-les-Moulineaux, France

${ }^{3}$ Lundbeck Research, Paramus, NJ

${ }^{4}$ Otsuka Pharmaceutical Development \&

Commercialization, Inc., Princeton, NJ

${ }^{5}$ H. Lundbeck A/S, Valby, Denmark

${ }^{6}$ Lundbeck LLC, Deerfield, IL

${ }^{7}$ Otsuka Pharmaceutical Europe, Wexham, UK

${ }^{8}$ Department of Psychiatry and Human Behavior, University of California, Irvine, CA

OBJECTIVE: The QUALIFY study directly compared the treatment effectiveness of the dopamine $D_{2}$ receptor partial agonist aripiprazole once-monthly $400 \mathrm{mg}$ (AOM 400) against the $D_{2}$ antagonist paliperidone palmitate once-monthly (PP) in stable patients with schizophrenia. The Heinrichs-Carpenter Quality-of-Life Scale (QLS) was the primary outcome, and Clinical Global Impression scores of illness severity (CGI-S) and improvement (CGI-I) were secondary effectiveness measures. In these post hoc analyses, changes in CGI scores after AOM 400 vs PP treatment and correlations between changes in QLS and CGI-S scores are presented.

METHODS: QUALIFY was a 28-week, randomized, openlabel, head-to-head study (NCT01795547) of 2 atypical long-acting injectable antipsychotics, AOM 400 and PP 
(flexible dosing with $78-234 \mathrm{mg} / \mathrm{month}$ as paliperidone palmitate). The primary endpoint was change from baseline to week 28 in QLS total score rated by a trained clinician blinded to treatment and analyzed using a mixed model for repeated measures (MMRM). Responders were defined as patients with either a decrease from baseline in CGI-S score of at least 1 or a CGI-I score $\leq 2$ at week 28. For both response definitions, the proportion of patients responding to AOM 400 and PP treatment was compared using logistic regression. Changes from baseline to week 28 for QLS and CGI-S scores were analyzed for correlation within MMRM for both treatment groups.

RESULTS: The analysis of the primary and secondary endpoints showed significant improvement for AOM 400 $(\mathrm{n}=136)$ vs PP $(\mathrm{n}=132)$. Least squares mean $(\mathrm{LSM})$ changes \pm SE in QLS total score from baseline to week 28 were $7.47 \pm 1.53$ for $\mathrm{AOM} 400$ and $2.80 \pm 1.62$ for PP, with an LSM difference between treatments of 4.67 (95\% CI: $[0.32,9.02], P=0.036)$. At week $28,62.7 \%$ (64/102) of patients on AOM 400 treatment had a decrease from baseline in CGI-S score of at least 1 compared with $43.5 \%(37 / 85)$ on PP treatment (adjusted odds ratio: $2.26,95 \%$ CI: [1.21, 4.20], $\mathrm{P}=0.010)$. At week $28,52.0 \%(53 / 102)$ of patients on AOM 400 treatment had a CGI-I score $\leq 2$ (much improved or very much improved) compared with $29.4 \%(25 / 85)$ on PP treatment (adjusted odds ratio: 2.51, 95\% CI: [1.36, 4.62], $P=0.0032$ ). The response on CGI-S was correlated with response on QLS after treatment for 28 weeks with AOM 400 (correlation estimate: $-0.59, P<0.0001, \mathrm{n}=143$ ) or PP (correlation estimate: $-0.38, P<0.0020, \mathrm{n}=136$ ).

CONCLUSIONS: In the QUALIFY study, AOM 400 showed superior improvements vs PP on health-related quality of life and functioning. The greater effectiveness of AOM 400 vs PP was supported by a higher number of patients responding to treatment, regardless of whether response was measured with CGI-S or CGI-I. After both AOM 400 and PP treatment, improvements in QLS were correlated with improvements in clinical symptoms measured with CGI-S, supporting the clinical relevance of QLS as a primary endpoint in schizophrenia.

FUNDING: This study was funded by Otsuka Pharmaceutical Development \& Commercialization, Inc.

\section{Effects of Aripiprazole Once-Monthly and Paliperidone Palmitate on Qls Domain Scores: Results from Qualify, A Randomized Head-To- Head Clinical Study}

Steven G. Potkin, $M D^{\prime}$; Jean-Yves Loze, MD, MSc ${ }^{2}$; Carlos Forray, $\mathrm{MD}^{3}$; Ross A. Baker, PhD, $\mathrm{MBA}^{4}$; Christophe Sapin, $\mathrm{MSc}^{5}$; Timothy Peters-Strickland, $\mathrm{MD}^{4}$; Aneta Fornal, PharmD ${ }^{4}$; Maud Beillat, MSc ${ }^{5}$;
Anna-Greta Nylander, PhD, MBA ${ }^{6}$; Peter Hertel, $P h D^{6}$, Henrik Steen Andersen, $\mathrm{MSc}^{6}$; Anna Eramo, $\mathrm{MD}^{7}$; Karina Hansen, $\mathrm{PhD}, \mathrm{MSc}^{5}$ and Dieter Naber, $\mathrm{MD}^{8}$

${ }^{1}$ Department of Psychiatry and Human Behavior, University of California, Irvine, CA

${ }^{2}$ Otsuka Pharmaceutical Europe, Wexham, UK

${ }^{3}$ Lundbeck Research, Paramus, NJ

${ }^{4}$ Otsuka Pharmaceutical Development \& Commercialization, Inc., Princeton, NJ

${ }^{5}$ Lundbeck SAS, Issy-les-Moulineaux, France

${ }^{6}$ H. Lundbeck A/S, Valby, Denmark

${ }^{7}$ Lundbeck LLC, Deerfield, IL

${ }^{8}$ University Medical Center Hamburg-Eppendorf, Hamburg, Germany

OBJECTIVES: Aripiprazole once-monthly $400 \mathrm{mg}$ (AOM 400) was compared head-to-head with paliperidone palmitate once-monthly (PP) over a 28-week period on the Heinrichs-Carpenter Quality-of-Life Scale (QLS), a validated measure of intrapsychic, interpersonal, and instrumental functioning in patients with schizophrenia. The present results are based on predefined secondary analyses of the effects of AOM 400 and PP on individual domains of the QLS.

METHODS: QUALIFY was a 28-week, open-label, raterblinded, head-to-head study (NCT01795547) of 2 atypical long-acting injectable antipsychotics, AOM 400 and PP (flexible dosing with $78-234 \mathrm{mg} / \mathrm{month}$ as paliperidone palmitate), to evaluate effectiveness in patients with schizophrenia, 18-60 years of age. Patients were randomized to 3 weeks' conversion to oral aripiprazole or paliperidone followed by 5 weeks' initiation of AOM 400 or PP treatment and continuing with injections every 4 weeks for 20 weeks. Mean a SD dose at week 24 was $387 \mathrm{a} 34 \mathrm{mg}$ AOM and 172ą52 $\mathrm{mg} \mathrm{PP}$ as paliperidone palmitate. The primary endpoint was change from baseline to week 28 in QLS total score analyzed with a mixed model for repeated measures (MMRM), and the study was powered accordingly. QLS total score comprises 21 items scored from 0 (severe impairment) to 6 (no impairment). In QUALIFY, QLS items were rated by a blinded clinician trained for consistent and reliable scoring. Items are grouped in 4 domains: interpersonal relations (8 items), instrumental role (4 items), intrapsychic foundations (7 items), and common objects and activities (2 items). In predefined MMRM analyses, changes from baseline on individual QLS domains were assessed.

RESULTS: The primary endpoint showed superior improvement with AOM 400 vs PP on the change in QLS total score from baseline to week 28 (least squares mean [LSM] difference between treatments, 4.67; 95\% CI: [0.32; 9.02], $\mathrm{P}=0.036$ ). Improvements in QLS domain scores from baseline to week 28 within the intrapsychic foundations domain were significantly greater after AOM 400 vs PP 
treatment (LSM difference: 1.8; 95\%CI: [0.09; 3.4], $\mathrm{P}=0.039$ ). Treatment differences in the remaining QLS domains tended to favor AOM 400 without reaching statistical significance vs PP (LSM differences: 1.8; 95\% CI: $[-0.14,3.7], \mathrm{P}=0.070$ for interpersonal relations; $0.92,95 \%$ CI: $[-0.28,2.1], \mathrm{P}=0.13$ for instrumental role; and $0.33,95 \%$ CI: $[-0.12,0.78], P=0.15$ for common objects and activities).

CONCLUSION: In a head-to-head study, superior clinicianrated improvement in health-related quality of life for AOM 400 vs PP was shown with QLS total score. The analysis of QLS domains suggests that improvements in the underlying intrapsychic foundations, such as sense of purpose, curiosity, empathy, ability to experience pleasure, and emotional interaction, may facilitate greater functioning with AOM 400 treatment compared with PP in patients with schizophrenia.

FUNDING: This study was funded by Otsuka Pharmaceutical Development \& Commercialization, Inc.

\section{Medication Interaction Online Tutorial: With Acronyms To Improve User Retention (MINOTAUR)}

Anthony Tobia, $\mathrm{MD}^{\prime}$ and Jason Minion, $M D^{\prime}$

${ }^{1}$ Rutgers Robert Wood Johnson Medical School, New Brunswick, NJ

ABSTRACT: Objective: The purpose of this study is to develop a clinical reference for drug interactions to be used by psychiatric residents in training. What differentiates our project from other medication interaction resources (e.g. Epocrates $($ ) $)$ is that we formatted the lists of drugs so as that the first letter of each name spells out an acronym. We believe these acronyms along with visual mnemonics also built into the program will result in improved retention of the material when compared to traditional resources.

METHODS: A psychopharmacology program was developed to promote understanding, retention, and future teaching of material on the $\mathrm{P} 450$ enzyme system. Isoenzymes 1A2, 2A6, 3A4, 3A5-7, 2B6, 2C8, 2C9, 2C18, 2C19, 2D6, 2E1 were presented using acronyms and visual mnemonics to enhance recall and retention. Two groups of residents stratified by post-graduate year $(\mathrm{n}=11)$ participated in the study and were asked to complete a questionnaire and 5-item quiz at baseline, 1 month, and 6 months. A Likert scale was used in the questionnaire to explore the views of the residents regarding learning, knowledge, and retention. Following baseline assessments, residents were presented the material in a PowerPoint format. The presentation was then uploaded to the university's intranet (psychiatry residents' forum), and residents were encouraged to reference the file during their clinical rotations. Residents completed follow-up assessments at 1- (recall) and 6-month (retention) intervals that included how often they used the program.

RESULTS: At baseline, both groups exhibited low quiz scores overall, each obtaining an average of one (out of five) question correct. First- and second-year residents scored higher on the follow-up quiz at 1 month (3.6 and 3.2 out of 5 questions correct, respectively). Scores representing retention of the material at 6 months were intermediate between the scores received before and after the material was presented, and correlated with the extent to which residents used the on-line reference.

CONCLUSIONS: The psychopharmacology program is an interactive method used to promote recall and retention of this challenging topic. Placing the material on the intranet forum allows the psychiatric residents to have the material available to them in various settings of their training to promote reinforcement. Future applications of this design should include easier access of the material than the institution's intranet allowed.

\section{Improvement in Secondary Effectiveness Measures with Aripiprazole Once-Monthly vs Paliperidone Palmitate in Qualify, A Head-To- Head Study}

Dieter Naber, $\mathrm{MD}^{\prime}$; Karina Hansen, PhD, $\mathrm{MSc}^{2}$; Carlos Forray, $\mathrm{MD}^{3}$; Ross A. Baker, PhD, $\mathrm{MBA}^{4}$; Christophe Sapin, $\mathrm{MSc}^{2}$; Maud Beillat, $\mathrm{MSc}^{2}$; Timothy PetersStrickland, $\mathrm{MD}^{4}$; Roland Larkin, $\mathrm{PhD}^{4}$; Anna-Greta Nylander, PhD, MBA ; Peter Hertel, PhD ; Henrik Steen Andersen, $\mathrm{MSc}^{5}$; Anna Eramo, MD ${ }^{6}$; Jean-Yves Loze, MD, MSc ${ }^{7}$ and Steven G. Potkin, $M D^{8}$

${ }^{1}$ University Medical Center Hamburg-Eppendorf, Hamburg, Germany

${ }^{2}$ Lundbeck SAS, Issy-les-Moulineaux, France

${ }^{3}$ Lundbeck Research, Paramus, NJ

${ }^{4}$ Otsuka Pharmaceutical Development \&

Commercialization, Inc., Princeton, NJ

${ }^{5}$ H. Lundbeck A/S, Valby, Denmark

${ }^{6}$ Lundbeck LLC, Deerfield, IL

${ }^{7}$ Otsuka Pharmaceutical Europe, Wexham, UK

${ }^{8}$ Department of Psychiatry and Human Behavior, University of California, Irvine, CA

OBJECTIVES: The effects of aripiprazole once-monthly 400 mg (AOM 400) and paliperidone palmitate once-monthly (PP) on health-related outcomes and clinical symptoms of schizophrenia were assessed in QUALIFY, a head-tohead clinical study in schizophrenia. 
METHODS: QUALIFY was a 28-week, open-label, raterblinded study (NCT01795547) comparing the atypical long-acting injectable antipsychotics, AOM 400 and PP (flexible dosing with $78-234 \mathrm{mg} / \mathrm{month}$ as paliperidone palmitate), in adult patients with schizophrenia, 18-60 years of age. Patients were randomized to 3 weeks conversion to oral aripiprazole or paliperidone followed by 5 weeks initiation of AOM 400 or PP treatment and continuing with injections every 4 weeks for 20 weeks. The primary endpoint showed noninferiority and subsequently superiority of AOM 400 compared with PP in change from baseline to week 28 on the rater-blinded Heinrichs-Carpenter Quality-of-Life Scale total score. Predefined secondary outcome measures of effectiveness are reported here, including the rater-blinded Investigator's Assessment Questionnaire (IAQ: assesses efficacy, safety, and tolerability of antipsychotic medications) total score at week 28 , and changes from baseline to week 28 on the Clinical Global Impression-Severity (CGI-S) and patient-rated Subjective Well-Being under Neuroleptic Treatment (short version) (SWN-S) scales. Secondary outcomes reported here were analyzed with a mixed model for repeated measures.

RESULTS: The study randomized 295 patients; 100/148 (67.6\%) in the AOM 400 group and 83/147 (56.5\%) in the PP group completed 28 weeks of treatment; at week 24 , mean ą SD dose was 387ą34 mg AOM and 172ą52 mg PP. In treated patients, baseline scores for CGI-S (AOM 400: 4.0ą0.66, PP: 4.0ą0.65) and SWN-S (AOM 400: 84.0ą16.1, PP: 85.3ą14.6) were similar between treatment groups. Improvements on CGI-S least square mean (LSM) change were found in the AOM $400 \quad(-0.75 \mathrm{a} 0.07)$ and PP (-0.46a 0.07$)$ groups, and improvements were greater with AOM 400 vs PP treatment (LSM difference: $-0.28,95 \%$ CI: $[-0.48,-0.09], \mathrm{P}=0.004)$. Treatment effectiveness measured with IAQ total score at week 28 also favored AOM 400 over PP (LSM: 32.32ą0.52 for AOM 400; 33.81ą0.55 for PP) with LSM differences for AOM 400 vs PP of -1.49 (95\% CI: [-2.94, -0.049], P = 0.043). Numerical improvements on the patient-reported SWN-S scale were observed in both treatment groups (LSM change from baseline: 4.82ą1.24 for AOM 400, 3.81ą1.30 for PP; LSM difference AOM 400 vs PP: 1.01, 95\% CI: [-2.40, 4.42], $\mathrm{P}=0.56)$.

CONCLUSIONS: In a head-to-head comparison, treatment with AOM 400 resulted in significantly greater improvements in health-related outcomes and clinical symptoms than PP in patients with schizophrenia. Secondary effectiveness measures in QUALIFY consistently favored treatment with AOM 400 and support the primary results demonstrating greater treatment effectiveness of $\mathrm{AOM}$ 400 compared with PP.

FUNDING: This study was funded by Otsuka Pharmaceutical Development \& Commercialization, Inc.
SSRI-Associated Tesd in Patients with WellTreated MDD After a Direct Switch to Vortioxetine or Escitalopram

Atul R. Mahableshwarkar, MD'; Paula L. Jacobsen, $M S^{\prime}$; Yinzhong Chen, PhD'; Wei Zhong, PhD'; Lambros Chrones, $M D^{\prime}$ and Anita H. Clayton, $M D^{2}$

${ }^{1}$ Takeda Development Center Americas, Deerfield, IL ${ }^{2}$ University of Virginia, Charlottesville, VA

BACKGROUND: Patients with MDD often report symptoms of sexual dysfunction despite being well treated for mood symptoms. Patients taking SSRIs are at greater risk for developing treatment-emergent sexual dysfunction (TESD), often switching treatment to manage this adverse event.

OBJECTIVE: This 8-wk, randomized, double-blind, headto-head comparison (NCT01364649) of vortioxetine and escitalopram examined the degree and severity of TESD in MDD patients taking SSRIs and the clinical impact of switching to vortioxetine or escitalopram.

METHODS: Well-treated MDD patients with SSRI-induced TESD were directly switched to vortioxetine $10 \mathrm{mg}$ $(\mathrm{n}=225)$ or escitalopram $10 \mathrm{mg}(\mathrm{n}=222)$ for wk 1 , and both treatments escalated to $20 \mathrm{mg}$ for wk 2 . Investigators could adjust the dose between 10 and $20 \mathrm{mg}$ at wks 4 and 6 . TESD was assessed at baseline (BL), wks 2, 4, 6, and 8 using the Changes in Sexual Functioning Questionnaire Short-Form (CSFQ-14), with higher scores indicating improved sexual functioning. Adverse events (AEs) were assessed at each study visit.

RESULTS: Patients taking citalopram (vortioxetine, 53.3\%; escitalopram, $51.8 \%)$, sertraline $(30.7 \%$; $34.7 \%)$, or paroxetine $(16.0 \% ; 13.5 \%)$ prior to randomization had welltreated depression (BL MADRS, 7.9; 8.3) with significant TESD (BL CSFQ-14, 36.5; 36.3), which was independent of age, gender, or current SSRI regimen. All patients were below the threshold (CSFQ-14 men $\leq 47$; women $\leq 41$ ) for healthy sexual functioning (BL CSFQ-14 men [39.9; 40.7]; women [33.5; 33.3]). 348 patients completed the study (vortioxetine, $\mathrm{n}=169 / 225$; escitalopram, $\mathrm{n}=179 / 222$ ). Patients switched to vortioxetine experienced superior improvement in TESD and a positive shift in sexual functioning compared to escitalopram (CSFQ-14 $\Delta+2.2$, $\mathrm{P}=0.013 ; \mathrm{MMRM})$. TESD symptoms improved in all patient groups, irrespective of previous SSRI. More patients who switched to vortioxetine improved from abnormal to healthy sexual functioning at wk 8, compared to escitalopram (113/217 [52.1\%]; 91/206 [44.2\%]; $\mathrm{P}=0.112)$. Antidepressant efficacy was maintained or slightly improved at wk 8 for both treatments and was independent of previous SSRI (MADRS, $\Delta-1.2 ;-1.5, \mathrm{P}=0.520$ ). The $\mathrm{AE}$ profile for vortioxetine was similar to previous trials, with nausea $(25.0 \%)$, headache $(9.4 \%)$, and dizziness $(8.0 \%)$ 
the most common TEAEs, and was similar across groups when evaluated by previous SSRI treatment, with the majority of AEs resolving after 14 days of treatment.

CONCLUSIONS: Patients treated with citalopram, paroxetine, or sertraline were safely and effectively switched to improve the symptoms of TESD. More vortioxetinetreated patients shifted to healthy sexual functioning compared to escitalopram, with the maintenance of clinical efficacy. Patients switched to vortioxetine demonstrated an $\mathrm{AE}$ profile similar to previous trials which was independent of their previous SSRI therapy.

FUNDING: This study was funded by Takeda Pharmaceutical Company, Ltd and H. Lundbeck A/S.

\section{Reduced Sexual Dysfunction with Aripiprazole Once-Monthly vs Paliperidone Palmitate: Results From Qualify, A Head-to-Head Study in Schizophrenia}

Steven G. Potkin, MD'; Jean-Yves Loze, MD, MSc ${ }^{2}$; Carlos Forray, $\mathrm{MD}^{3}$; Ross A. Baker, PhD, MBA ${ }^{4}$; Christophe Sapin, $\mathrm{MSc}^{5}$; Timothy Peters-Strickland, $\mathrm{MD}^{4}$; Maud Beillat, MSc ; Phyllis Salzman, $P h D^{4}$; Anna-Greta Nylander, PhD, MBA ${ }^{6}$; Peter Hertel, $P h D^{6}$; Henrik Steen Andersen, $M S c^{6}$; Anna Eramo, $\mathrm{MD}^{7}$; Karina Hansen, $\mathrm{PhD}, \mathrm{MSc}^{5}$ and Dieter Naber, $\mathrm{MD}^{8}$

${ }^{1}$ Department of Psychiatry and Human Behavior,

University of California, Irvine, CA

${ }^{2}$ Otsuka Pharmaceutical Europe, Wexham, UK

${ }^{3}$ Lundbeck Research, Paramus, NJ

${ }^{4}$ Otsuka Pharmaceutical Development \&

Commercialization, Inc., Princeton, NJ

${ }^{5}$ Lundbeck SAS, Issy-les-Moulineaux, France

${ }^{6}$ H. Lundbeck A/S, Valby, Denmark

${ }^{7}$ Lundbeck LLC, Deerfield, IL

${ }^{8}$ University Medical Center Hamburg-Eppendorf, Hamburg, Germany

OBJECTIVE: The QUALIFY study directly compared the treatment effectiveness of the dopamine $\mathrm{D}_{2}$ receptor partial agonist aripiprazole once-monthly $400 \mathrm{mg}$ (AOM 400) to the $\mathrm{D}_{2}$ antagonist paliperidone palmitate once-monthly $(\mathrm{PP})$ on health-related quality of life in patients with schizophrenia. Here we present post hoc analyses of incidences of sexual dysfunction and safety data related to levels of prolactin.

METHODS: QUALIFY was a 28-week, randomized, openlabel, head-to-head study (NCT01795547) of 2 atypical long-acting injectable anti-psychotics (LAIs), AOM 400 and PP (flexible dosing, per label, with 78-234 mg/month as paliperidone palmitate) in schizophrenia. Included patients were aged 18-60 years, needing a change from current oral antipsychotic treatment and, in the judgment of the investigator, would benefit from LAI treatment. The
Arizona Sexual Experience Scale (ASEX) is a 5-item, 6-point, patient-rated scale used to identify individuals with sexual dysfunction. The presence of sexual dysfunction was pre-defined as an ASEX total score of $>=19$, or a score of $>=5$ on any item, or a score of $>=4$ on at least 3 items. The odds ratio (OR) for sexual dysfunction after 28 weeks of AOM 400 or PP treatment was calculated post hoc using logistic regression adjusted for baseline presence or absence of sexual dysfunction. Serum prolactin was measured by a central laboratory at baseline and last visit and summarized with descriptive statistics.

RESULTS: Primary endpoint showed superior improvement with AOM $400(\mathrm{n}=136)$ vs PP $(\mathrm{n}=132)$ on change from baseline to week 28 on Heinrichs-Carpenter Quality-of-Life Scale (QLS) total score. Patients had lower odds for sexual dysfunction after 28 weeks of AOM 400 (baseline: 56/103; week 28: 39/103, 37.9\%) vs PP (baseline: 55/84; week 28: 53/84, 63.1\%) treatment (adjusted OR: 0.29; 95\%CI: [0.14$0.61], \mathrm{P}=0.0012$ ). The difference between treatments was significant in males (adjusted OR: 0.33; 95\%CI: [0.13-0.86], $\mathrm{P}=0.023$ ) and in females (adjusted OR: 0.14; 95\%CI: [0.03-0.62], $\mathrm{P}=0.0099)$. In the AOM 400 group, mean \pm SD serum prolactin decreased from $401 \pm 629 \mathrm{mIU} / \mathrm{L}$ at baseline $(\mathrm{n}=139)$ to $156 \pm 323 \mathrm{mIU} / \mathrm{L}$ at week 28 $(\mathrm{n}=99)$ while an increase from $489 \pm 823 \mathrm{mIU} / \mathrm{L}$ ( $\mathrm{n}=135)$ at baseline to $1043 \pm 1006 \mathrm{mIU} / \mathrm{L}$ at week 28 ( $n=81$ ) was observed in the PP group. The pattern in mean changes from baseline to last visit persisted in both sexes. Six patients (all PP-treated) exhibited prolactinrelated treatment-emergent adverse events.

CONCLUSIONS: Superior improvements in health-related quality of life on the clinician-rated QLS suggested greater effectiveness for AOM 400 vs PP, and the improvements were accompanied by a lower risk for sexual dysfunction with AOM 400 vs PP treatment. The effects of AOM 400 and PP on prolactin and rates of sexual dysfunction may be related to the differential effect of the compounds at the dopamine $\mathrm{D}_{2}$ receptor.

FUNDING: This study was funded by Otsuka Pharmaceutical Development \& Commercialization, Inc.

\section{Metabolic Syndrome Status in Patients with Schizophrenia After Short-Term Treatment with Lurasidone, Olanzapine, and Quetiapine Xr: A Pooled Analysis}

John W. Newcomer, $\mathrm{MD}^{\prime}$; Michael Tocco, PhD ${ }^{2}$; Andrei Pikalov, MD, PhD ; Hanzhe Zheng, PhD'; Josephine Cucchiaro, $P h D^{2}$ and Antony Loebel, $M D^{2}$

${ }^{1}$ Florida Atlantic University Charles E. Schmidt College of Medicine, Boca Raton, FL

${ }^{2}$ Sunovion Pharmaceuticals Inc. 
STUDY OBJECTIVE: To evaluate the status of metabolic syndrome in patients with schizophrenia following shortterm treatment with lurasidone, olanzapine, and quetiapine XR.

METHODS: Patient-level data were pooled from three phase 3, randomized, double-blind, placebo-controlled, 6-week studies of fixed-dose lurasidone $(40-160 \mathrm{mg} / \mathrm{d})$ in the treatment of patients with an acute exacerbation of schizophrenia. Two studies included an active comparator (olanzapine $15 \mathrm{mg} / \mathrm{d}$ or quetiapine XR $600 \mathrm{mg} / \mathrm{d}$ ). Patients were classified as having metabolic syndrome (based on the updated US National Cholesterol Education Program criteria [without using drug treatment criteria]) if they met any 3 of the following 5 criteria: 1 ) elevated waist circumference ( $\geq 102 \mathrm{~cm}$ for men, $\geq 88 \mathrm{~cm}$ for women), 2) elevated triglycerides ( $\geq 150 \mathrm{mg} / \mathrm{dL}), 3$ ) reduced high-density lipoprotein cholesterol $(<40 \mathrm{mg} / \mathrm{dL}$ in men, $<50 \mathrm{mg} / \mathrm{dL}$ in women), 4) elevated blood pressure (systolic $\geq 130 \mathrm{~mm} \mathrm{Hg}$ or diastolic $\geq 85 \mathrm{~mm} \mathrm{Hg}$ ), and 5) elevated fasting glucose $(\geq 100 \mathrm{mg} / \mathrm{dL})$. Logistic regression analysis adjusting for baseline metabolic syndrome status using a last observation carried forward (LOCF) approach was performed.

RESULTS: The analysis included 1203 patients with both baseline and post-baseline data for metabolic syndrome (lurasidone, $\mathrm{n}=703$; olanzapine, $\mathrm{n}=105$; quetiapine $\mathrm{XR}, \mathrm{n}=96$; placebo, $\mathrm{n}=299$ ). At baseline, the percentage of patients with metabolic syndrome was $19.1 \%$ for placebo, $21.6 \%$ for lurasidone, $21.0 \%$ for olanzapine, and $14.6 \%$ for quetiapine XR. Rates of metabolic syndrome at Week 6 study endpoint (LOCF) were $20.1 \%$ for placebo, $21.8 \%$ for lurasidone, $36.2 \%$ for olanzapine, and $38.5 \%$ for quetiapine $\mathrm{XR}(\mathrm{P}<0.001$ for olanzapine vs placebo and quetiapine $\mathrm{XR}$ vs placebo). In patients without metabolic syndrome at baseline, the proportion who met criteria for metabolic syndrome after 6 weeks of treatment (LOCF) was $10.7 \%$ of patients receiving placebo, $9.4 \%$ of lurasidone-treated patients, $25.3 \%$ of olanzapine-treated patients, and $30.5 \%$ of quetiapine XR $(\mathrm{P}<0.05$ for olanzapine vs placebo; $\mathrm{P}<0.01$ for quetiapine XR vs placebo).

CONCLUSIONS: This post hoc pooled analysis in patients with schizophrenia found statistically significant differences between lurasidone and other atypical antipsychotic agents in the percentage of patients who developed metabolic syndrome after short-term therapy (6 weeks). These patients were significantly less likely to develop metabolic syndrome during treatment with lurasidone compared with olanzapine or quetiapine XR.

FUNDING: This study was sponsored by Sunovion Pharmaceuticals Inc.
ClinicalTrials.gov identifiers:

NCT00549718, NCT00615433, and NCT00790192.

REFERENCES:

Newcomer JW, Tocco M, Pikalov A, Zheng H, Cucchiaro J, Loebel A. Metabolic syndrome prevalence in patients with schizophrenia receiving short-term treatment with lurasidone, olanzapine, and quetiapine XR: a pooled analysis. Presented at the American Society of Clinical

Psychopharmacology Annual Meeting; June 22-25, 2015; Miami, FL.

\section{Venlafaxine Induced Neutropenia}

Maria Mojica-Rangel, MSN, APRN, PMHNP-BC'; Palanikumar Gunasekar, $M B B S^{2}$; Vithyalakshmi Selvaraj, $M D^{3}$ and Imad Alsakaf, $M D^{3}$

${ }^{1}$ Creighton University School of Medicine, Omaha, NE

${ }^{2}$ Department of Biomedical Sciences, Creighton

University, Omaha, NE

${ }^{3}$ Department of Psychiatry, Creighton University

School of Medicine, Omaha, NE

ABSTRACT: Venlafaxine is a serotonin-norepinephrine reuptake inhibitors (SNRI), antidepressant approved by Food and Drug Administration for the treatment of major depression, generalized anxiety disorder, social anxiety disorder, and panic disorder. We here report a case of a patient who experienced leukopenia and neutropenia after initiating treatment with venlafaxine. To the best of our knowledge, there are two published case reports in which venlafaxaine was possibly causing a decrease in WBC and/or neutrophil count.

\section{Tardive Dyskinesia in The Era of Second} Generation Antipsychotics: A Case Report and Literature Review

Shirshendu Sinha, $M B B S^{1,2}$; Ajay Shah, MD ${ }^{1,3}$ and Sylvester Edeh, $M D^{\prime}$

${ }^{1}$ Department of Psychiatry, University of Connecticut School of Medicine, Farmington, CT

${ }^{2}$ Department of Psychiatry and Psychology, Mayo Clinic College of Medicine, Rochester, MN

${ }^{3}$ Menninger Department of Psychiatry and Behavioral Sciences, Baylor College of Medicine, Houston, TX

PURPOSE: Tardive dyskinesia (TD) is a delayed onset iatrogenic movement disorder of various phenomenology caused by dopamine receptor-blocking agents. Newer Second Generation Antipsychotics (SGAs) are thought to have lower TD risk. Here we report a case of lurasidone-induced TD. The goal of this case report is to alert providers to continue to be diligent in monitoring for TD in patients taking SGAs.

CLINICAL CASE: A 33-year-old white woman with history of bipolar disorder, generalized anxiety disorder, 
post-traumatic stress disorder, presented with symptoms of low mood, irritability, decreased interest, poor concentration, and low energy. She denied mania or psychosis. She had been on lithium carbonate $300 \mathrm{mg}$ twice daily (BID), clonidine $0.1 \mathrm{mg}$ BID and lorazepam $0.5 \mathrm{mg}$ BID as needed (PRN) for anxiety. Her most recent serum lithium level was 0.4. She was kept on lithium and clonidine with lurasidone $20 \mathrm{mg}$ added to target bipolar depression, the dose of which was titrated up to $60 \mathrm{mg}$ at bedtime (HS) over several months. Benztropine was added for prophylaxis for extrapyramidal symptoms (EPS). She was then lost to follow-up and stopped medications for two months. Upon her return she was restarted on lurasidone $40 \mathrm{mg}$ HS and lithium 300 $\mathrm{mg}$ HS for bipolar depression with clonazepam $0.5 \mathrm{mg}$ daily PRN for anxiety and benztropine $0.5 \mathrm{mg}$ daily for EPS prophylaxis. Lithium and benztropine were discontinued due to nausea and double vision. Clonazepam was stopped due to poor response. Eszopiclone was started for sleep and gabapentin for anxiety. Lurasidone was titrated up to $60 \mathrm{mg}$ HS. She developed involuntary tongue movements 9 weeks after lurasidoe re-challenge. A diagnosis of TD was suspected and lurasidone was tapered off over the next few weeks. She was restarted on clonazepam $0.5 \mathrm{mg}$ BID, this time for TD. Her dyskinetic movements disappeared 10 weeks following discontinuation of lurasidone. Her medications at that time included: ramelteon $8 \mathrm{mg}$ for sleep, clonazepam $0.5 \mathrm{mg}$ BID for TD, oxcarbazepine $600 \mathrm{mg}$ BID for mood swings and gabapentin $300 \mathrm{mg}$ BID with $600 \mathrm{mg}$ at HS for anxiety and sleep.

DISCUSSION: Pathophysiology of TD is poorly understood. Theories include dopamine-2 receptor hypersensitivity, oxidative stress created from chronic antipsychotic use, dysfunctional striatal GABA input to motor neurons, and lower expression of serotonin (5HT-2A) receptors. There is no Food and Drug Administration approved drug for the treatment of TD. In our case, TD symptoms remitted 10 weeks after discontinuation of lurasidone. Clinically this case exemplifies early identification of TD and discontinuation of offending agent as potentially reversing the course of TD. Clonazepam contributed to symptom resolution most likely due to increased striatal GABAergic input. Additionally, gabapentin and ramelteon may have augmented the therapeutic benefit of clonazepam through GABAergic and anti-oxidant effects, respectively.

\section{Treatment of Acute Schizophrenia in Adult}

Patients Using Either Brexpiprazole (OPC-34712) or Aripiprazole, an Exploratory Study

Leslie Citrome, $\mathrm{MD}, \mathrm{MPH}^{1} ; \mathrm{Ai}$ Ota, $\mathrm{BSc}^{2} ;$ Kazuhiro Nagamizu, BSc ${ }^{2}$; Pamela Perry, $M S^{3}$; Emmanuelle Weiller, PsyD $D^{4}$ and Ross A. Baker, PhD, $M B A^{3}$

${ }^{1}$ New York Medical College, Valhalla, NY

${ }^{2}$ Otsuka Pharmaceutical Co., Ltd., Tokyo, Japan
${ }^{3}$ Otsuka Pharmaceutical Development \& Commercialization, Inc., Princeton, NJ

${ }^{4}$ H. Lundbeck A/S, Valby, Denmark

BACKGROUND: Brexpiprazole is a serotonin-dopamine activity modulator that acts as a partial agonist at serotonin 5-HT1A and dopamine D2 receptors at similar potency, and as an antagonist at serotonin 5-HT2A and noradrenaline alpha1B/2C receptors. Brexpiprazole has lower intrinsic activity at the D2 receptor than aripiprazole, suggesting a lower potential to induce D2 agonist-mediated adverse events such as akathisia, insomnia, restlessness, and nausea.

STUDY OBJECTIVE: The objective of this open-label study was to explore the effects of 6-weeks' treatment with brexpiprazole or aripiprazole in patients with schizophrenia (NCT02054702).

METHODS: Patients with an acute relapse of schizophrenia were randomized to receive brexpiprazole 1 to $4 \mathrm{mg}$ /day (3 mg/day target dose) or aripiprazole 10 to $20 \mathrm{mg} /$ day (15 mg/day target dose) for 6 weeks. The mean change in Positive and Negative Syndrome Scale (PANSS) total score from baseline to Week 6 was analyzed.

RESULTS: A total of 97 patients were treated with brexpiprazole $(\mathrm{N}=64)$ or aripiprazole $(\mathrm{N}=33)$. A reduction in the symptoms of schizophrenia as assessed by the PANSS total score were observed in both treatment groups (least square mean change at Week 6: -22.9 and -19.4 for brexpiprazole and aripiprazole, respectively). Brexpiprazole was well tolerated and the incidence of extrapyramidal symptom (EPS)-related adverse events including akathisia was lower in the patients treated with brexpiprazole $(14.1 \%)$ than in the patients treated with aripiprazole $(30.3 \%)$.

CONCLUSION: Clinically relevant improvements in psychopathology were observed in patients with acute schizophrenia treated with brexpiprazole or aripiprazole. Brexpiprazole was well tolerated with a lower incidence of EPS-related adverse events than aripiprazole.

FUNDING: This study was funded by Otsuka Pharmaceutical Development \& Commercialization, Inc., Princeton, NJ, USA, Otsuka Pharmaceutical Co., Ltd. Tokyo, Japan and H. Lundbeck A/S, Valby, Denmark.

\section{Switching from Inadequate Adjunctive Treatment Options to Brexpiprazole Adjunctive to Antidepressant: An Open-Label Study}

Ross A. Baker, PhD, MBA'; Takao Okame, $\mathrm{MSc}^{2}$; Pamela Perry, MS ; Yuki Matsushima, MEng ${ }^{2}$ and Emmanuelle Weiller, PsyD ${ }^{3}$

${ }^{1}$ Otsuka Pharmaceutical Development \&

Commercialization, Inc., Princeton, NJ 
${ }^{2}$ Otsuka Pharmaceutical Co., Ltd., Tokyo, Japan

${ }^{3}$ H. Lundbeck A/S, Valby, Denmark

BACKGROUND: Two pivotal studies have evaluated the efficacy, tolerability and safety of brexpiprazole, a serotonin-dopamine activity modulator, as adjunctive treatment in patients with major depressive disorder.

STUDY OBJECTIVE: This study investigated the effect of brexpiprazole on depressive symptoms in patients inadequately responsive to augmentation of their current antidepressants with other adjunctive therapies (NCT02012218).

METHODS: A 6-week open-label, single-arm study evaluated switching from other adjunctive treatments to brexpiprazole adjunctive to antidepressants. Patients (18-65 years) had inadequate response to a medication adjunctive to their base antidepressant (aripiprazole, quetiapine, bupropion, other antidepressants, or stimulants), and previous failure to an adequate dose and duration of antidepressant monotherapy. Efficacy was measured by change from baseline in Montgomery-Åsberg Depression Rating Scale (MADRS) total score and Cognitive and Physical Functioning Questionnaire (CPFQ). Safety and tolerability were assessed.

RESULTS: Of 61 enrolled patients, 51 (83.6\%) completed 6 weeks' treatment. Mean baseline MADRS and CPFQ scores were 29.6 and 29.3 respectively, and similar across prior adjunctive therapies. Mean change from baseline for MADRS was -17.3 $(p<0.0001)$, ranging from -12.8 $(p<0.0001)$ for patients switched from adjunctive aripiprazole $(\mathrm{n}=12)$ to $-19.5(p<0.0001)$ for patients switched from combination antidepressants $(n=12)$. Mean change from baseline for CPFQ was $-9.2(p<0.0001)$, ranging from $-5.6(\mathrm{p}=.0178)$ for patients switched from combination antidepressant $(\mathrm{n}=12)$ to $-13.3 \quad(\mathrm{p}=0.0003)$ for patients switched from adjunctive stimulants $(n=6)$. Fatigue was the most common treatment emergent adverse event, reported by $>10 \%$ of patients $(9 / 61$, $14.8 \%$ ).

CONCLUSION: Patients switched to brexpiprazole showed marked improvement in depressive symptoms and selfreported improvements in cognitive and physical functioning. Brexpiprazole was well tolerated.

FUNDING: This study was funded by Otsuka Pharmaceutical Development \& Commercialization, Inc., Princeton, NJ, USA, Otsuka Pharmaceutical Co., Ltd. Tokyo, Japan and H. Lundbeck A/S, Valby, Denmark.
Adjunctive Brexpiprazole (OPC-34712) in Patients with Major Depressive Disorder And Irritability: An Exploratory Study

François Menard, $\mathrm{MD}^{\prime}$; Maurizio Fava, $\mathrm{MD}^{2}$; Charlotte Kampp Davidsen, $\mathrm{MSc}^{\prime}$; Anna Eramo, $\mathrm{MD}^{3}$ and Ross A. Baker, $P h D, M B A^{4}$

${ }^{1}$ H. Lundbeck A/S, Valby, Denmark

${ }^{2}$ Massachusets General Hospital, Boston, MA

${ }^{3}$ Lundbeck LLC, Deerfield, IL

${ }^{4}$ Otsuka Pharmaceutical Development \&

Commercialization, Inc., Princeton, NJ

BACKGROUND: Brexpiprazole is a serotonin-dopamine activity modulator that is a partial agonist at serotonin 5-HT1A and dopamine D2 receptors with similar potency, and an antagonist at 5-HT2A and noradrenaline alpha1B/2C receptors.

STUDY OBJECTIVE: The objective of this study was to evaluate adjunctive treatment with brexpiprazole on irritability symptoms in patients with major depressive disorder (MDD) who demonstrated inadequate response to antidepressant treatment (ADT) (NCT01942785).

METHODS: Patients with MDD and inadequate response to $\mathrm{ADT}$ were treated with their current ADT for a period of 2 weeks. Patients who still had an inadequate response, and were irritable (Inventory of Depressive Symptomatology (IDS)-C30 item $6>2$ ), received 6 weeks openlabel treatment with their current ADT at the same dose and adjunctive treatment with brexpiprazole. Brexpiprazole was discontinued at Week 6 , and patients continued with their current ADT up to Week 10. Several patientrated and clinician-rated instruments assessing irritability, hostility, impulsivity and anger, as well as depressive symptoms were analyzed for changes from Baseline to Week 6, and changes between Week 6 and Week 10.

RESULTS: A total of 54 patients were treated with ADT and adjunctive brexpiprazole. At Week 6, improvements from baseline irritability symptoms were observed assessed by Sheehan Irritability Scale (SIS) total score (-21.1) and SIS Item 1 (-3.5), as well as Kellner Symptom Questionnaire anger-hostility subscore (-7.7). More patients stopped having anger attacks during treatment (15 patients) than patients developing anger attacks during treatment (5 patients), measured by the Anger Attacks Questionnaire (AAQ). Depressive symptoms also improved at Week 6 (MADRS total score: -14.2). Irritability symptoms moderately worsened after brexpiprazole discontinuation assessed at Week 10. Adjunctive brexpiprazole was well tolerated, and no new safety concerns were observed.

CONCLUSION: Adjunctive treatment with brexpiprazole may represent a novel strategy for the treatment of 
irritability symptoms in patients with MDD and inadequate response to $\mathrm{ADT}$.

FUNDING: This study was funded by Otsuka Pharmaceutical Development \& Commercialization, Inc., Princeton, NJ, USA and H. Lundbeck A/S, Valby, Denmark.

\section{Investigation of Treatment Emergent Specific}

Sexual Dysfunction in Adult Male Patients Taking Antipsychotic Medications: A Cross Sectional Study

Shirshendu Sinha, $M B B S^{\prime}$; Shirin Schilling, $M D^{\prime}$; Grace Chan, PhD'; Myra Saha, DO'; Galina Prpich, $M S^{\prime}$; Victoria Scranton, $M S^{\prime}$; Pantea Farahmand, $M D^{\prime}$; Ronak Jhaveri, $M D^{\prime}$ and Jayesh Kamath, $M D, P h D^{\prime}$

${ }^{1}$ Department of Psychiatry, University of Connecticut School of Medicine, Farmington, CT

${ }^{2}$ Department of Psychiatry and Psychology, Mayo Clinic College of Medicine, Rochester, MN

BACKGROUND: Sexual side effects are twice as common in patients taking antipsychotics compared to healthy controls and are a major determinant of treatment non adherence. The evidence is inconclusive that antipsychotic medication induced hyper-prolactinemia (HPRL) (serum prolactin $(\mathrm{PRL})>20 \mathrm{ng} / \mathrm{ml}$ ) is the primary cause of sexual dysfunction (SD). Severe hyperprolactinemia (SHPRL) (serum prolactin $>35 \mathrm{ng} / \mathrm{ml}$ ) in men is primarily associated with reduced sexual desire. HPRL might also indirectly inhibit erectile function by inhibiting the production of testosterone (T). We seek to utilize while "The Antipsychotics and Sexual Functioning Questionnaire" (ASFQ) to capture changes in sexual functioning and association with antipsychotic medication initiation.

OBJECTIVES: To explore treatment emergent SD in adult males, especially reduced sexual desire and erectile dysfunction, with validated sexual function questionnaires and to examine if there is an association with HPRL/SHPRL and other endocrine aberrations.

METHODS: This ongoing naturalistic cross sectional study conducted at the University of Connecticut outpatient and inpatient psychiatric facilities includes males with bipolar and schizophrenia spectrum disorders, age 18-65 years old, who have been taking antipsychotic medication for at least 8 weeks. Data collected include demographics, clinical characteristics, self-reported and clinician administered sexual side effect scales. Laboratory data were obtained for serum prolactin, testosterone and thyroid stimulating hormone (TSH).

RESULTS: Nine males participated in the study at time of preliminary analysis. Demographics include mean age: 49 , ethnicity: $100 \%$ white/non-Hispanic, marital status: $89 \%$ single, education: $\mathbf{7 8 \%}$ undergraduate. Primary psychiatric diagnosis included schizophrenia $(22 \%)$, schizoaffective disorder $(22 \%)$, and bipolar spectrum disorders (44\%). Eleven antipsychotics were used (73\% second generation). By self-reported Arizona Sexual Experience Scale (ASEX), $44 \%$ reported sexual side effects. On clinician administered ASFQ, more than $50 \%$ complained of reduced sexual desire and erectile dysfunction consistent with expected SD associated with SHPRL. Prolactin levels ranged from 5.5 to 32.92 , testosterone level ranged from $100-529$, and TSH ranged from 0.48-5.31.

CONCLUSION: Despite small sample size, there is discrepancy for SD between self-report and clinician administered questionnaires; the latter appeared to be more reliable in capturing subtle changes in sexual experience in patients taking antipsychotic medications. The common SDs in males treated with antipsychotic medication are hypoactive sexual desire and erectile dysfunction. The outcomes from this ongoing pilot study will help us design a larger, longitudinal study to decipher pathophysiology of antipsychotic medication induced sexual dysfunction with goal of improved medication compliance and treatment outcomes, and enhanced quality of life.

\section{Adjunctive Brexpiprazole (OPC-34712) in Patients with Major Depressive Disorder and Sleep Disturbances: An Exploratory Study \\ Andrew Krystal, $\mathrm{MD}^{\prime}$; Aurelia Mittoux, PhD ${ }^{2}$; Peter Meisels, $M S c^{2}$ and Ross A. Baker, PhD, $M B A^{3}$ \\ ${ }^{1}$ Duke University Hospital, Durham, NC \\ ${ }^{2}$ H. Lundbeck A/S, Valby, Denmark \\ ${ }^{3}$ Otsuka Pharmaceutical Development \& \\ Commercialization, Inc., Princeton, NJ}

BACKGROUND: Brexpiprazole, a serotonin-dopamine activity modulator, is a partial agonist at serotonin 5-HT1A and dopamine D2 receptors with similar potency, and an antagonist at 5-HT2A and noradrenaline alpha1B/2C receptors. Study Objective: The objective was to evaluate the effects of adjunctive treatment with brexpiprazole on sleep disturbance parameters in patients with MDD inadequately responsive to antidepressant treatment (ADT) (NCT01942733).

METHODS: Patients with an inadequate response to ADT continued treatment with their current ADT for a period of 2-weeks. Patients who still had an inadequate response, and experienced sleep disturbances, received 8-week open-label treatment with their current ADT and adjunctive brexpiprazole ( 1 to $3 \mathrm{mg} /$ day)

RESULTS: A total of 44 patients were treated with ADT and adjunctive brexpiprazole. At week 8, improvements 
from baseline, as measured by polysomnography (PSG; $\mathrm{n}=41$ ) and the Consensus Sleep Diary for Morning (CSD-M; $\mathrm{n}=21$ ), respectively, were observed in Sleep Efficiency (10.4\% and $13.4 \%$; observed cases [OC]), Total Sleep Time (49.0 min and $69.9 \mathrm{~min}$; OC), Sleep Onset Latency (-19.7 min and -37.1 min; OC), and WakeTime after Sleep Onset (-26.4 and -42.9, OC). Improvements were also demonstrated in the Insomnia Severity Index (ISI) total score $(-9.2, \mathrm{n}=41$; mixed model repeated measures [MMRM]), alertness as measured by the Epworth Sleepiness Scale (ESS) total score (-2.1, $\mathrm{n}=41$; MMRM) and functioning as measured by the Cognitive and Physical Functioning Questionnaire (CPFQ; -8.4, $\mathrm{n}=41$; MMRM). No new safety concerns were observed compared to previous brexpiprazole studies.

CONCLUSION: Adjunctive treatment with brexpiprazole may represent a novel strategy for treatment of sleep disturbances in patients with MDD and inadequate response to $\mathrm{ADT}$.

FUNDING: This study was funded by Otsuka Pharmaceutical Development \& Commercialization, Inc., Princeton, NJ, USA and H. Lundbeck A/S, Valby, Denmark.

\section{The Effect of Brexpiprazole (OPC-34712) in Adult Outpatients with Early-Episode Schizophrenia: An Exploratory Study}

Ashok Malla, MD'; Pamela Perry, MS ${ }^{2}$; Kazuhiro Nagamizu, BSc ${ }^{3}$; Emmanuelle Weiller, Psy $D^{4}$ and Ross A. Baker, $P h D, M B A^{2}$

\footnotetext{
${ }^{1}$ Department of Psychiatry, McGill University, Montreal, Canada

${ }^{2}$ Otsuka Pharmaceutical Development \&

Commercialization, Inc., Princeton, NJ

${ }^{3}$ Otsuka Pharmaceutical Co., Ltd., Tokyo, Japan

${ }^{4}$ H. Lundbeck A/S, Valby, Denmark
}

BACKGROUND: Treatment during the early stages of schizophrenia should ideally prevent residual and persistent symptoms, without tolerability trade-offs. This could facilitate adequate social functioning.

STUDY OBJECTIVE: This open-label study (NCT02013622) investigated the effects of flexibly dosed brexpiprazole, a serotonin-dopamine activity modulator, in early-episode schizophrenia.

METHODS: Relatively stable outpatients aged 18-35 years with early-episode schizophrenia $(<5$ years since start of first episode at study start; Positive and Negative Syndrome Scale [PANSS] Total score $<80$ ) were enrolled and received open-label brexpiprazole up to $4 \mathrm{mg} /$ day, for 16 weeks. During the first 2- to 4-week period, brexpiprazole was cross-titrated with previous antipsychotic medications. Assessments included: change from baseline to week 16 in PANSS Total Score, PANSS prosocial items, Clinical Global Impression-Severity (CGI-S) score, Personal and Social Performance (PSP) scale, Scale for Level of Functioning in Schizophrenia (SLOF) and the Pittsburg Sleep Quality Index (PSQI); and Clinical Global Impression-Improvement (CGI-I) score at Weeks 1-16. Safety and tolerability were also assessed.

RESULTS: Overall, 49 patients received brexpiprazole and $25(51 \%)$ patients completed 16 weeks of treatment. Improvements were observed from baseline to Week 16 in PANSS total score (least square mean change: -10.2, $\mathrm{p}<.0001)$, PANSS prosocial items $(\mathrm{p}<.0001)$ and modified PANSS prosocial items $(p<.0001)$. CGI-S and CGI-I scores supported these findings. An improvement of social function as seen in PSP $(p=0.0003)$ and SLOF $(p=0.0002)$ scales and of sleep as measured by PSQI $(p=0.0177)$ was observed. Brexpiprazole was well tolerated with safety results comparable to previous pivotal trials.

CONCLUSION: Brexpiprazole may represent a novel and effective treatment strategy for patients with earlyepisode schizophrenia, and may facilitate improvement of social function in relatively stable patients.

FUNDING: This study was funded by Otsuka Pharmaceutical Development \& Commercialization, Inc., Princeton, NJ, USA, Otsuka Pharmaceutical Co., Ltd. Tokyo, Japan and H. Lundbeck A/S, Valby, Denmark.

\section{Aripiprazole Once-Monthly and Paliperidone Palmitate in Patients with Schizophrenia Stratified by Age: Results from Qualify, a Head- to-Head Study}

Dieter Naber, $M D^{\prime} ;$ Karina Hansen, PhD, $M S c^{2}$; Carlos Forray, $\mathrm{MD}^{3}$; Ross A. Baker, PhD, $\mathrm{MBA}^{4}$; Christophe Sapin, $\mathrm{MSc}^{2}$; Maud Beillat, $\mathrm{MSc}^{2}$; Timothy Peters-Strickland, $\mathrm{MD}^{4}$; Anna-Greta Nylander, PhD, $\mathrm{MBA}^{5}$; Peter Hertel, PhD ${ }^{5}$; Henrik Steen Andersen, $\mathrm{MSc}^{5}$; Anna Eramo, $\mathrm{MD}^{6}$; Jean-Yves Loze, MD, MSc and Steven G. Potkin, $M D^{8}$

${ }^{1}$ University Medical Center Hamburg-Eppendorf, Hamburg, Germany

${ }^{2}$ Lundbeck SAS, Issy-les-Moulineaux, France

${ }^{3}$ Lundbeck Research, Paramus, NJ

${ }^{4}$ Otsuka Pharmaceutical Development \&

Commercialization, Inc., Princeton, NJ

${ }^{5}$ H. Lundbeck A/S, Valby, Denmark

${ }^{6}$ Lundbeck LLC, Deerfield, IL

${ }^{7}$ Otsuka Pharmaceutical Europe, Wexham, UK

${ }^{8}$ Department of Psychiatry and Human Behavior, University of California, Irvine, CA 
OBJECTIVES: The QUALIFY study directly compared the effectiveness of aripiprazole once-monthly $400 \mathrm{mg}$ (AOM $400)$ to paliperidone palmitate once-monthly (PP) in patients with schizophrenia. The effects of AOM 400 and PP treatment in patients $<=35$ years and $>35$ years were assessed in prespecified secondary analyses.

METHODS: QUALIFY was a 28-week, randomized, openlabel, head-to-head study (NCT01795547) of 2 atypical long-acting injectable antipsychotics, AOM 400 and PP (flexible dosing with $78-234 \mathrm{mg} / \mathrm{month}$ as paliperidone palmitate) in patients with schizophrenia, ages 18-60 years; week 24 mean doses \pm SD were $387 \pm 34 \mathrm{mg}$ AOM and $172 \pm 52 \mathrm{mg}$ PP as paliperidone palmitate. The primary endpoint was change from baseline to week 28 on the Heinrichs-Carpenter Quality-of-Life Scale (QLS) total score analyzed with a mixed model for repeated measures in the full analysis set (FAS). Key secondary outcomes included Investigator's Assessment Questionnaire (IAQ) and Clinical Global Impression-Severity of Illness (CGI-S). QLS and IAQ were assessed by raters blinded to the treatment. Higher QLS scores and lower IAQ and CGI-S scores indicate better clinical condition. Preplanned assessments were conducted in the FAS (AOM 400: $\mathrm{n}=136$, PP: $\mathrm{n}=132$ ) in patients $<=35$ years (AOM 400: $\mathrm{n}=41, \mathrm{PP}: \mathrm{n}=37$ ) and $>35$ years (AOM 400: $\mathrm{n}=95$, PP: $\mathrm{n}=95)$.

RESULTS: The primary analysis showed superiority of AOM 400 vs PP on the change in QLS total score with a least squares mean (LSM) difference between treatments of 4.67 (95\% CI: [0.32, 9.02], $\mathrm{P}=0.036)$. Mean \pm SD baseline QLS total scores were similar between groups; AOM 400: $66.0 \pm 21.7$, PP: $62.9 \pm 21.5$. The LSM treatment difference in the change in QLS total scores significantly favored AOM 400 over PP in patients $<=35$ years $(10.68,95 \%$ CI: $[0.70,20.66], \mathrm{P}=0.037)$ with $\mathrm{LSM}$ changes $( \pm \mathrm{SE})$ from baseline to week 28 of $9.34 \pm 3.16$ for $\mathrm{AOM} 400$ and $-1.34 \pm 3.14$ for PP. Significant improvements with AOM 400 over PP in patients $<=35$ years were also found in total IAQ score at week 28 (LSM treatment difference: -2.65, 95\% CI: [-5.28, -0.02], $\mathrm{P}=0.048$ ) and in change from baseline to week 28 in CGI-S scores (LSM treatment difference: $-0.44,95 \%$ CI: $[-0.83,-0.06], \mathrm{P}=0.026)$. In patients $>35$ years, nonsignificant LSM treatment differences numerically favored AOM 400 over PP on QLS (2.81, 95\% CI: [-2.02, 7.63], $\mathrm{P}=0.25$, with LSM changes $\pm \mathrm{SE}$ in QLS of $6.54 \pm 1.71$ for $\mathrm{AOM} 400$ and $3.74 \pm 1.74$ for PP). LSM treatment differences for IAQ $(-1.02,95 \%$ CI: [-2.77, 0.73], $\mathrm{P}=0.25)$ and CGI-S $(-0.22,95 \%$ CI: [-0.44, 0.01 ], $\mathrm{P}=0.061$ ) also favored AOM 400.

CONCLUSION: AOM 400 showed superiority over PP on health-related quality of life as measured by the QLS in the total study population. In patients $<=35$ years, greater improvements with AOM 400 vs PP were seen consistently across effectiveness outcomes, suggesting that AOM 400 treatment at a younger age may confer more beneficial treatment outcomes than PP in patients with schizophrenia.

FUNDING: This study was funded by Otsuka Pharmaceutical Development \& Commercialization, Inc.

\section{Adjunctive Brexpiprazole (OPC-34712) in Patients with Major Depressive Disorder and Anxiety Symptoms: An Exploratory Study}

Lori Davis, $\mathrm{MD}^{1,2}$; Ai Ota, BSc ${ }^{3}$; Pamela Perry, $M S^{4}$; Kana Tsuneyoshi, BS ; Anna Eramo, $M D^{4}$ and Ross $A$. Baker, $P h D, M B A^{5}$

${ }^{1}$ Veterans Affairs Medical Center, Tuscaloosa, AL

${ }^{2}$ University of Alabama School of Medicine,

Birmingham, AL

${ }^{3}$ Otsuka Pharmaceutical Co., Ltd., Tokyo, Japan

${ }^{4}$ Lundbeck LLC, Deerfield, IL

${ }^{5}$ Otsuka Pharmaceutical Development \&

Commercialization, Inc., Princeton, NJ

BACKGROUND: Anxiety symptoms, common in patients with major depressive disorder (MDD), are associated with greater severity, impaired functioning, and less-favorable outcomes. Brexpiprazole, a serotonin-dopamine activity modulator, is a partial agonist at 5-HT1A and dopamine $\mathrm{D} 2$ receptors at similar potency, and an antagonist at 5-HT2A and noradrenaline alpha1B/2C receptors.

STUDY OBJECTIVE: This open-label study explored effects of adjunctive brexpiprazole in patients with MDD and anxiety symptoms (NCT02013531).

METHODS: Patients with MDD and anxiety symptoms (Hamilton Anxiety Rating Scale $[\mathrm{HAM}-\mathrm{A}] \geq 20$ ) with inadequate response to current $\mathrm{ADT}$ were enrolled and received open-label $\mathrm{ADT}+$ brexpiprazole $1-3 \mathrm{mg} /$ day ( $2 \mathrm{mg}$ /day target dose) for 6 weeks. Efficacy endpoints included change in clinician-rated Montgomery-Åsberg Depression Rating Scale (MADRS) and HAM-A total score (baseline to Week 6) and change in the 92-item patient-rated Kellner Symptom Questionnaire (KSQ, range 0-92) total score (baseline to Week 6).

RESULTS: A total of 37 patients were treated with brexpiprazole $+\mathrm{ADT}$; of these, 32 patients completed 6 weeks of treatment. Mean MADRS total score at baseline was 30.3, mean HAM-A total score was 27.0, and mean KSQ total score was 55.9, indicating patients had moderate to severe symptoms of depression and anxiety. Improvements were observed in MADRS total score (least square [LS] mean change) from Baseline to Week 6 in patients treated with brexpiprazole + ADT (LS mean change: -19.6 , 95\% CI [-22.7;-16.6]) and in HAM-A total score (LS mean change: -17.8, 95\% 
CI $[-20.3 ;-15.3])$. In addition, mean change from Baseline to Week 6 in KSQ total score (-29.4) also improved. Adjunctive brexpiprazole was well tolerated; the incidence of activating adverse events (akathisia, restlessness, agitation, anxiety, and insomnia) was low $(\leq 5 \%)$; no clinically relevant changes in the mean laboratory test values, vital signs, or electrocardiogram parameter values were observed.

CONCLUSION: Adjunctive treatment with brexpiprazole may represent a novel and effective strategy for treatment of patients with MDD and symptoms of anxiety showing an inadequate response to $\mathrm{ADT}$.

FUNDING: This study was funded by Otsuka Pharmaceutical Development \& Commercialization, Inc., Princeton, NJ, USA, Otsuka Pharmaceutical Co., Ltd. Tokyo, Japan and H. Lundbeck A/S, Valby, Denmark.

\section{Efficacy and Safety of Lurasidone in Older Adults with Bipolar Depression}

Martha Sajatovic, $M D^{\prime}$; Brent Forester, $M D, M S c^{2,3}$; Joyce Tsai, PhD ${ }^{4}$; Hans Kroger, MPH, MS ; Andrei Pikalov, MD, PhD ${ }^{4}$; Josephine Cucchiaro, $P h D^{4}$ and Antony Loebel, $\mathrm{MD}^{4}$

${ }^{1}$ Case Western Reserve University School of Medicine, Cleveland, $\mathrm{OH}$

${ }^{2}$ Harvard Medical School, Boston, MA

${ }^{3}$ McLean Hospital, Belmont, MA

${ }^{4}$ Sunovion Pharmaceuticals, Inc, Marlborough, MA and Fort Lee, NJ

OBJECTIVE: Treatment of bipolar disorder in the elderly, especially longer-term, has not been well-studied. The aim of this post-hoc analysis was to evaluate the efficacy and tolerability of up to 7.5 months of lurasidone in older adults (age $\geq 55$ years) with bipolar depression.

METHODS: The older adult sample that was analyzed was a subgroup of patients with bipolar I depression who were randomized, double-blind, to 6 weeks of monotherapy with lurasidone $(20-60 \mathrm{mg} / \mathrm{d}$ or $80-120 \mathrm{mg} / \mathrm{d})$ vs. placebo $(\mathrm{N}=83 / 485 ; 17.1 \%)$ or lurasidone $(20-120$ $\mathrm{mg} / \mathrm{d})$ vs. placebo, each adjunctive with $\mathrm{Li}$ or VPA ( $\mathrm{N}=53 / 340 ; 15.6 \%$ ). The primary outcome was change in MADRS at week 6. Patients who completed acute treatment were eligible for treatment with lurasidone 20 $120 \mathrm{mg} / \mathrm{d}(\mathrm{N}=55 / 316 ; 17.4 \%)$ in a 6 month, open-label study; concomitant mood stabilizers were permitted.

RESULTS: At Week 6, mean change in MADRS was significantly greater for lurasidone $20-60 \mathrm{mg} \quad(-15.4$; $\mathrm{P}<0.01$; effect size $=0.86)$ and $80-120 \mathrm{mg} \quad(-14.1$; $\mathrm{P}<0.02$; effect size $=0.74)$ vs placebo $(-7.1)$. Adjunctive therapy with lurasidone (vs placebo) was associated with numerically greater improvement at Week 6 in MADRS $(-13.9$ vs. -11.1 ; ns; effect size $=0.26)$. Discontinuation rates due to adverse events, for lurasidone vs placebo, were similar for the monotherapy $(6.8 \%$ vs $6.9 \%)$ and adjunctive therapy $(3.8 \%$ vs $7.1 \%)$ studies. At the end of 6 months of open-label treatment with lurasidone, monotherapy and adjunctive therapy, respectively, minimal changes were observed in the older adult population in mean weight $(-1.0 \mathrm{~kg} ;-0.4 \mathrm{~kg})$ and median total cholesterol $(-2.0 \mathrm{mg} / \mathrm{dL}$; $+6.0 \mathrm{md} / \mathrm{dL})$, triglycerides $(+2.5 \mathrm{mg} / \mathrm{dL} ;+6.0 \mathrm{mg} / \mathrm{dL})$, and HbA1c $(0.0 \% ;-0.1 \%)$. Patients continued to improve on the MADRS during 6 months of lurasidone treatment in both the monotherapy (-10.1) and adjunctive therapy (-10.1) groups.

CONCLUSIONS: The results of these post-hoc analyses found lurasidone to be an effective acute treatment for older adults with bipolar depression. Efficacy of lurasidone in this age group was maintained over 6 months of continued treatment, with minimal effects on weight or metabolic parameters.

FUNDING: This study was funded by Sunovion Pharmaceuticals Inc.

Clinicaltrials.gov identifier: NCT00868452; NCT00868699; NCT00868959.

\section{Lurasidone in Bipolar Depression: Acute Stable Response as a Predictor of Long-Term Treatment Response}

Dan Iosifescu, $\mathrm{MD}^{\prime}$; Joyce Tsai, PhD ${ }^{2}$; Andrei Pikalov, $M D, P h D^{2}$; Hans Kroger, $M P H, M S^{2}$ and Antony Loebel, $M D^{2}$

${ }^{1}$ Icahn School of Medicine at Mount Sinai, New York, NY

${ }^{2}$ Sunovion Pharmaceuticals Inc., Fort Lee, NJ and Marlborough, MA

OBJECTIVE: To evaluate the predictive value of acute treatment response on long-term response at 6 months, in patients treated with lurasidone as monotherapy.

METHODS: This post-hoc observed cases analysis included patients with bipolar I depression who completed 6 weeks of double-blind, placebo-controlled treatment with lurasidone (fixed-flexible doses of $20-60 \mathrm{mg} / \mathrm{d}$ and 80-120 mg/d; dosages combined) followed by 6 months of open-label treatment with lurasidone (flexible doses of $20-120 \mathrm{mg} / \mathrm{d})$. Response was defined as $\geq 50 \%$ reduction from double-blind baseline on the Montgomery Åsberg Depression Rating Scale (MADRS) total score. Acute stable response was defined as 2 consecutive weeks of meeting response criteria during initial the 6 weeks of treatment, and partial response was defined as $\geq 25 \%$ but 
$<50 \%$ reduction on the MADRS. The proportion of responders at 6 months was determined among patients who were non-responders, partial responders, and responders at each week during weeks 2-6. The predictive value of acute stable response at any time during the initial 6 week treatment and at weeks 5-6 specifically, for long-term response at 6 months, was evaluated with sensitivity, specificity, positive predictive value (PPV), negative predictive value (NPV), and area under the ROC (receiver operative characteristic) curve (AUC). Additionally, we determined the proportion of responders at 6 months with initial improvement in CGIBP-S (clinical global impression of severity, bipolar depression) $\geq 1$ during weeks 1-6 of acute treatment.

RESULTS: The majority of patients who completed 6 months of open-label lurasidone treatment following 6 weeks of acute double-blind lurasidone treatment were responders at endpoint, regardless of their response status at weeks 2-6. Among non-responders, partial responders, and responders at 6 weeks, the proportion of responders at the end of 6 months of extension treatment was $84 \%(16 / 19), 79 \%(30 / 38)$, and $96 \%$ (93/97), respectively. The ability of 2 consecutive weeks of acute response to predict long-term response at month 6 was similar, regardless of whether acute stable response occurred at any time during weeks 1-6 (sensitivity, 61\%; specificity, 80\%; PPV, 97\%; NPV, 18\%; AUCROC, $0.776)$, or stable response occurred specifically at weeks 5-6 (sensitivity, 58\%; specificity, 87\%; PPV, 98\%; NPV, 18\%; AUCROC, 0.788). 139 patients completed 6 months of extension treatment, of whom $94 \%$ experienced improvement in CHI-BP-S $\geq 1$ point during the 6 weeks of initial double-blind treatment with lurasidone.

CONCLUSIONS: Based on the AUCROC, the predictive value of acute stable response was fair-to-moderate. Most patients who responded after 6 months of treatment had demonstrated clinically meaningful improvement by the end of initial acute treatment. The majority of responders and partial responders at the end of acute treatment achieved full response at the end of extension treatment.

FUNDING: This study was funded by Sunovion Pharmaceuticals Inc.

\section{Lurasidone in the Treatment of Bipolar}

\section{Depression: Effect of Baseline Depression}

\section{Severity on Clinical Outcome}

Andrei Pikalov, MD, PhD'; Joyce Tsai, PhD'; Josephine Cucchiaro, $P h D^{\prime}$ and Antony Loebel, $M D^{\prime}$

${ }^{1}$ Sunovion Pharmaceuticals Inc., Marlborough, MA and Ft. Lee, NJ
OBJECTIVE: The aim of this post-hoc analysis was to evaluate the impact of baseline depression severity on clinical response in patients with bipolar depression treated with lurasidone.

METHODS: Patients with bipolar I depression in 2 registration trials were randomized to 6 weeks of double-blind, placebo-controlled treatment with lurasidone monotherapy $(20-60 \mathrm{mg} / \mathrm{d}$ or $80-120 \mathrm{mg} / \mathrm{d}$; Study $1 ; \mathrm{N}=499$ ); or lurasidone adjunctive to lithium or valproate $(20-120 \mathrm{mg} / \mathrm{d}$; Study $2 ; \mathrm{N}=345$; required serum levels were 0.6-1.2 mEq/liter for lithium and 50$125 \mathrm{mg} / \mathrm{ml}$ for valproate). Two baseline depression severity groups were defined post-hoc: a moderate $(\mathrm{MADRS}=20-29)$ and a high $(\mathrm{MADRS} \geq 30)$ severity group. For each group, changes in MADRS total and CGI-BP-S scores were analyzed using a mixed model for repeated measures analysis. Additional efficacy outcomes included the 16-Item Quick Inventory of Depressive Symptomatology (QIDS-SR16).

RESULTS: In the monotherapy study, 214/499 (42.9\%) of patients were in the moderate severity group (mean MADRS $=26.0)$ and 285/499 $(57.1 \%)$ were in the high severity group $(\mathrm{MADRS}=33.9)$; in the adjunctive therapy study, $137 / 345(39.7 \%)$ of patients were in the moderate severity group (mean MADRS $=25.7$ ) and $208 / 345(60.3 \%)$ were in the high severity group (MADRS $=34.0$ ). In the monotherapy study, lurasidone effect sizes (d) for MADRS change at week 6 in the high severity vs moderate severity groups were $d=0.60$ $(\mathrm{P}<0.001)$ vs $0.40(\mathrm{P}=0.035)$ for the $20-60 \mathrm{mg} / \mathrm{d}$ dose range, and $\mathrm{d}=0.55(\mathrm{P}=0.002)$ vs $0.50(\mathrm{P}=0.008)$ for the $80-120 \mathrm{mg} / \mathrm{d}$ dose range. Monotherapy with lurasidone was associated with significantly greater week 6 improvement for both severity groups on the CGI-BP-S (effect sizes, 0.42-0.68), and on the QIDS-SR16 (effect sizes, 0.39-0.59). In the adjunctive therapy study, lurasidone effect sizes for MADRS change in the high severity vs moderate severity groups were $\mathrm{d}=0.25$ $(\mathrm{P}=0.10) \quad$ vs $\mathrm{d}=0.41 \quad(\mathrm{P}=0.033)$; effect sizes for CGI-BP-S change were $\mathrm{d}=0.21(\mathrm{P}=0.18)$ vs $\mathrm{d}=0.60$ $(\mathrm{P}=0.002)$; and effect sizes for QIDS-SR16 change were $\mathrm{d}=0.32(\mathrm{P}=0.026)$ vs $\mathrm{d}=0.73(\mathrm{P}<0.001)$. Lurasidone was well-tolerated in both studies, regardless of depression severity subgroup.

CONCLUSIONS: In this post-hoc analysis, monotherapy with lurasidone was associated with significant improvement, with moderate-to-large effect sizes, for both moderate and high depression severity subgroups. Adjunctive therapy with lurasidone was also associated with significant improvement in the moderate severity subgroup, with moderate-to-large effect sizes. However, the treatment effect of adjunctive lurasidone was notably smaller in the high severity subgroup. The reasons for 
this are uncertain, but may be attributable, in part, to treatment with lithium or valproate in the placebo group, resulting in greater placebo improvement in MADRS and CGI-BP-S scores compared with the monotherapy study.

Clinicaltrials.gov identifier: NCT00868699, NCT00868452 Sponsored by Sunovion Pharmaceuticals Inc.

\section{The Complex Multimodal Management of First- Psychotic Episode in Patients with Morphometric Alteration in Hippocampal Formation}

Bashkim Kadriu, $\mathrm{MD}^{\prime}$; Sonia Demetrios, $\mathrm{MD}^{\prime}$, Wen Gu, PhD'; Gautam Mirchandani, $\mathrm{MD}^{2}$; Marilena Jennings, $M D^{\prime}$ and J. Arturo Sanchez-Lacay, $M D, M P H^{\prime}$

${ }^{1}$ Albert Einstein College of Medicine, Bronx, NY

${ }^{2}$ Department of Radiology, Bronx-Lebanon Hospital Center, Bronx, NY

ABSTRACT: Research data in schizophrenia suggests that early life brain structural alterations are present in subjects at high risk of developing psychosis or at first psychotic break. Changes in medial temporal lobe, specifically hippocampal formation (HP/F) reduction were consistently reported in first psychotic episode as well as mood-anxiety, seizures, and neurodegenerative disorders. In attempt to better understand the treatment complexity of first psychotic episodes, we present three adolescent inpatient cases, each presenting with first episode of psychosis that was associated with significant alterations of hippocampal formation.

CASE 1. A 16-year-old male who presented with emotional and perceptual disturbances associated with GAD and severe panic attacks. The MRI was pertinent with decreased right HP/F. Psychological assessment showed borderline range cognitive functioning and physiological anxiety. Patient was started on risperidone, gradually titrated to therapeutic dose of $1.5 \mathrm{mg}$ po bid. Frequent panic attacks were treated with lorazepam on prn basis. Patient responded gradually to medication titration and once the psychosis subsided, the panic attacks disappeared.

CASE 2. A 17-year-old female who presented with psychosis, mood lability and catatonic symptoms further complicated by myoclonic seizure activity. The patient also had significant history of cannabis use. MRI showed decreased left HP/F and increased bilateral fissure. The sleep deprived EEG studies showed idiopathic myoclonic generalized seizure. Psychological testing demonstrated global deficits in cognitive functioning, poor reality testing and insight. The patient was started on lorazepam $2 \mathrm{mg}$ bid for catatonic symptoms and risperidone titrated to a therapeutic dose of $1 \mathrm{mg}$ in the morning and $2 \mathrm{mg}$ at bedtime for symptoms of psychosis. The patient's psychosis improved with treatment and catatonia subsided with lorazepam.

CASE 3. A 17-year-old male who presented with frank paranoia, persecutory delusions, active auditory/visual hallucinations, coupled with increasing unprovoked agitated behavior in the context of heavy substance use dependence. The MRI showed bilateral HP/F reduction. Psychological assessment showed low average cognitive functioning, poor reality testing and deficits in visual memory. He was started on quetiapine $50 \mathrm{mg}$ orally, cross-titrated to risperidone $0.5 \mathrm{mg}$ reaching a therapeutic dose of $2 \mathrm{mg}$ bid. With treatment, his symptoms of disorganized behavior, frank paranoia, and delusions gradually improved.

DISCUSSION: Numerous studies have found early morphometric and functional hippocampal changes in patients with schizophrenia. Our clinical case presentations and $\mathrm{HP} / \mathrm{F}$ alteration are consistent with the hypothesis that in schizophrenia there is early disruption/arrest of normal neurogenesis that may contribute to the gray matter reduction in multiple brain areas including $\mathrm{HP} / \mathrm{F}$ that likely starts during early childhood and/or adolescence.

\section{The Metabolic Tolerability Profile of Adjunct Brexpiprazole (OPC-34712) in Major Depressive Disorder}

Craig Nelson, $\mathrm{MD}^{\prime}$; Aleksandar Skuban, $\mathrm{MD}^{2}$; Mary Hobart, PhD ${ }^{2}$; Peter Zhang, PhD ${ }^{2}$; Catherine Weiss, $P h D^{2}$ and Emmanuelle Weiller, $P s y D^{3}$

${ }^{1}$ University of California San Francisco, San Francisco, CA

${ }^{2}$ Otsuka Pharmaceutical Development \&

Commercialization, Inc., Princeton, NJ

${ }^{3}$ H. Lundbeck A/S, Valby, Denmark

BACKGROUND: Some atypical antipsychotics are associated with metabolic adverse effects, including weight gain, impaired glucose metabolism and dyslipidemia. Brexpiprazole is a rationally designed serotonindopamine activity modulator that is a partial agonist at 5-HT1A and dopamine D2 receptors at similar potency, and an antagonist at $5 \mathrm{HT} 2 \mathrm{~A}$ and noradrenaline alpha1B/2C receptors.

STUDY OBJECTIVE: The metabolic profile of brexpiprazole was evaluated in patients (pts) with major depressive disorder (MDD), based on data from two phase III studies (NCT01360645 and NCT01360632) and two large longterm (LT) open-label studies (NCT01360866 and NCT01447576).

METHODS: In two short-term (ST) studies, pts with MDD and inadequate response to antidepressants (ADTs) were 
enrolled and received single-blind ADT for 8 weeks. Pts with inadequate response after this prospective phase were randomized to ADT + brexpiprazole or ADT + placebo for 6 weeks. Both studies included fixed doses (2mg; $1 \mathrm{mg}$ and $3 \mathrm{mg}$ ). The LT studies were open-label, 52weeks, flexible-dose $(0.5-3 \mathrm{mg} /$ day and $0.25-3 \mathrm{mg} /$ day $)$ studies with brexpiprazole + ADT. The LT studies enrolled de novo pts, pts who had completed one of the two pivotal studies, as well as pts who had completed one of two phase II studies. Metabolic parameters included weight, glucose, and lipid metabolism-related laboratory measurements.

RESULTS: In the ST studies, mean change in weight from baseline to Week 6 was $1.4 \mathrm{~kg}, 1.4 \mathrm{~kg}, 1.6 \mathrm{~kg}$, and $0.3 \mathrm{~kg}$ for the $1 \mathrm{mg}, 2 \mathrm{mg}$, $3 \mathrm{mg}$ brexpiprazole $+\mathrm{ADT}$ and placebo + ADT groups, respectively. In the LT study, the mean change in weight from baseline to Week 52 was $3.1 \mathrm{~kg}$. An increase in weight of $\geq 7 \%$ at any visit was seen in $4.9 \%, 4.8 \%$, and $2.2 \%$ of brexpiprazole + ADT $1 \mathrm{mg}$, $2 \mathrm{mg}$, and $3 \mathrm{mg}$ pts vs $1.9 \%$ of placebo + ADT pts in the ST studies and in $29.5 \%$ of the pts treated with brexpiprazole $+\mathrm{ADT}$ in the LT study. For fasting metabolic parameters, mean changes from baseline to last visit in the ST studies were (brexpiprazole + ADT 1mg, 2mg, and $3 \mathrm{mg}$ vs placebo $+\mathrm{ADT})$ : total cholesterol 0.70, 2.60, and 1.49 vs $-0.06 \mathrm{mg} / \mathrm{dL}$; high-density lipoprotein cholesterol $1.12,1.21$, and 2.07 vs $0.56 \mathrm{mg} / \mathrm{dL}$; low-density lipoprotein cholesterol $-1.50,1.37$, and -0.77 vs $-1.18 \mathrm{mg} / \mathrm{dL}$; triglycerides $3.21,-0.83$, and $2.20 \mathrm{vs}-2.27 \mathrm{mg} / \mathrm{dL}$; and glucose $-0.75,-0.40$, and 0.70 vs $0.92 \mathrm{mg} / \mathrm{dL}$. Mean changes from baseline to Week 52 in the LT studies were: total cholesterol $0.12 \mathrm{mg} / \mathrm{dL}$; HDL cholesterol $-3.21 \mathrm{mg} /$ $\mathrm{dL}$; LDL cholesterol $0.26 \mathrm{mg} / \mathrm{dL}$; triglycerides $16.91 \mathrm{mg} /$ $\mathrm{dL}$; and glucose $4.61 \mathrm{mg} / \mathrm{dL}$.

CONCLUSION: A moderate weight increase was observed on brexpiprazole with no clinically relevant changes in lipid profiles or other metabolic parameters observed. Results from the LT study were consistent with those of the ST pivotal studies and confirmed the good tolerability of brexpiprazole.

FUNDING: This study was funded by Otsuka Pharmaceutical Development \& Commercialization, Inc., Princeton, NJ, USA and H. Lundbeck A/S, Valby, Denmark.

\section{Effect of Vortioxetine on Functional Capacity in Patients with Major Depressive Disorder with Self-Reported Cognitive Dysfunction}

William Jacobson, $P h D^{\prime}$; Christina Kurre Olsen, $P h D^{2}$; Atul R. Mahableshwarkar, $M D^{\prime}$; Yinzhong Chen, $P h D^{\prime}$ and Richard S.E. Keefe, $P h D^{3}$

${ }^{1}$ Takeda Development Center Americas, Deerfield, IL

${ }^{2}$ H. Lundbeck A/S, Copenhagen, Denmark
${ }^{3}$ Duke University Medical Center, Department of Psychiatry and Behavioral Sciences, Durham, NC

OBJECTIVE: Evaluate the impact of flexible-dose vortioxetine 10-20mg (VOR) vs placebo (PBO) on objective and subjective measures of patient functionality after 8 weeks of treatment in adults with MDD (NCT01564862). Duloxetine 60mg (DUL) was included as an active reference for assay sensitivity of depressive symptoms.

METHODS: Adults with moderate to severe MDD (18-65yrs, MADRS $\geq 26$ ) and self-reported cognitive dysfunction (ie, difficulty concentrating, slow thinking, difficulty in learning new things) were enrolled in this placebo-controlled, active reference study. Objective performance-based aspects of functional capacity were evaluated using UCSD Performance-Based Skills Assessment (UPSA), and subjective aspects with Cognitive and Physical Functioning Questionnaire (CPFQ) and Work Limitation Questionnaire (WLQ). Path analysis assessed the proportion of functional improvement attributed to a direct treatment effect, not due to improvements in mood.

RESULTS: 602 patients were randomized (VOR, $\mathrm{n}=198$; PBO, $\mathrm{n}=194$; DUL, $\mathrm{n}=210)$. VOR demonstrated a significant improvement in UPSA composite score vs $\mathrm{PBO}$ at week $8(\mathrm{n}=175, \Delta+2.94, \mathrm{P}<0.001$; ANCOVA, OC), UPSA-B $(\mathrm{n}=97, \Delta+4.02, \mathrm{P}=0.001)$ but not UPSA-VIM $(\mathrm{n}=78, \Delta+1.75, \mathrm{P}=0.078)$. DUL did not demonstrate a significant change in UPSA composite score $(\mathrm{n}=187, \Delta+0.38, \mathrm{P}=0.637)$, UPSA-B $(\mathrm{n}=93$, $\Delta-0.35, \mathrm{P}=0.775)$, or UPSA-VIM $(\mathrm{n}=94, \Delta+1.17$, $\mathrm{P}=0.219)$ vs $\mathrm{PBO}$ at week 8. Path analysis of the UPSA composite score showed that $96.9 \%$ of the effect of VOR on performance-based functional capacity was direct and not mediated by improvement in depressive symptoms. VOR improved work productivity in working patients vs $\mathrm{PBO}$, as assessed by change in WLQ time management subscale score at week 8 $(\mathrm{VOR} \mathrm{n}=72, \Delta-8.13, \mathrm{P}=0.045 ;$ DUL $\mathrm{n}=72, \Delta-2.53$, $\mathrm{P}=\mathrm{NS} ;$ ANCOVA, OC). Neither VOR nor DUL separated from placebo in the other WLQ subscale scores or the percentage of productivity loss score; however, VOR demonstrated a decrease vs PBO in output demands score $(\mathrm{n}=70, \Delta-7.60, \mathrm{P}=0.069)$. Path analysis showed a direct effect of VOR on work productivity (time, 45.9\%; output demands, 53.2\%) with improvements on mental demands primarily an indirect effect $(68.7 \%)$, mediated by improvements in depressive symptoms. Patients with a baseline CPFQ > 25 (predefined cut-off for clinically relevant patient-perceived cognitive symptoms) demonstrated a significant improvement in mean CPFQ score for VOR and DUL vs PBO $(\mathrm{n}=135, \Delta-1.7, \quad \mathrm{P}=0.041 ; \mathrm{n}=140, \Delta-1.8$, 
$\mathrm{P}=0.024 ;$ MMRM, FAS) which was largely an indirect effect $(70.4 \% ; 89.6 \%)$.

CONCLUSIONS: Treatment with VOR significantly improved scores on the performance-based assessments of patient functionality. Path analyses suggest that the performance-based improvements for VOR are primarily a direct treatment effect and not due to improvements in mood. These data suggest that VOR produces improved functional capacity in patients with MDD.

FUNDING: This study was funded by Takeda Pharmaceutical Company, Ltd. \& H. Lundbeck A/S.

\section{A Case of Fluoxetine Induced Paranoia?}

William Levitt, $M D^{\prime}$ and Rajesh Gaddam, $M B B S^{\prime}$

${ }^{1}$ Bergen Regional Medical Center, Paramus, NJ

ABSTRACT: We present the case of a 28-year-old Korean female who presented to the outpatient psychiatric clinic for complaints of depression and anxiety. The patient had no history of psychosis or paranoia upon arrival. The patient then began treatment with fluoxetine for the next three months with varying compliance. After beginning treatment with fluoxetine, she began to develop paranoia that people were talking about her at work. The paranoia then worsened with an increase in her fluoxetine dosage from 10 to $20 \mathrm{mg}$.

While there are a number of hypotheses for the neurobiology of schizophrenia, less is known about the mechanism of paranoia. However, paranoia is believed to be a result of regulation of serotonin, dopamine, norepinephrine and GABA transmission-especially in the ventral basal ganglia. ${ }^{1}$

Fluoxetine is one of the most commonly prescribed antidepressants. Fluoxetine has been shown to work via 5HT-2C antagonism, which results in increased dopamine and norepinephrine levels via modulating release and reuptake. ${ }^{1,2}$

Increased dopamine has been known to cause paranoia and suspicious thinking in addition to overt psychosis. Increased dopamine release in the prefrontal cortex may possibly alter that patient's interpretation of reality, thus resulting in her paranoia. ${ }^{3,4}$ Excess dopamine in the mesolimbic pathway has also been connected to the development of positive symptoms such as paranoia seen in our patient.

Fluoxetine may also result in the development of paranoia via norepinephrine modulation. It has been hypothesized that norepinephrine plays a significant role in the development of paranoia and has been implicated in schizophrenia. ${ }^{5}$

Fluoxetine appears to work via various mechanisms stimulating serotonin, dopamine and norepinephrine levels. As such, paranoia as result of fluoxetine treatment is a rare complication that we believe needs to be addressed as little is known.

\section{REFERENCES:}

1. Lauterbach E. Serotonin Reuptake Inhibitors, Paranoia, and the Ventral Basal Ganglia. Clin Neuropharmacol. 1991; 14(6): 547-555.

2. Karlsson H, Hietala J, Saarijarvi S. Acute paranoid-hallucinatory psychosis after an overdose of fluoxetine and enalapril: a case report. Nord J Psychiatry. 1998; 52: 153-154.

3. Jordan S, Kramer GL, Zukas PK, Moeller M, Petty F. In vivo biogenic amine efflux in medial prefrontal cortex with imipraminen, fluoxetine, and fluvoxamine. Synapse. 1994; 18: 294-297.

4. Tanda G, Carboni E, Frau R, Di Chiara G. Increase of extracellular dopamine in the prefrontal cortex: a trait of drugs with antidepressant potential? Psychopharmacology (Berl). 1994; 115: 285-288.

5. Fitzgerald P. Is elevated norepinephrine an etiological factor in some cases of schizophrenia? Psychiatry Res. 2014; 215(3): 497-504.

\section{Using Transdermal Administration of Psychiatric Medications to Enhance Quality of Life in An Elderly Nursing Home Patient Diagnosed with Dementia and Depression: A Case Study}

\section{Kathleen T Patterson, Ph.D., PMHNP-BC}

Deerfield Behavioral Health, Warren, PA

STUDY OBJECTIVES: When elderly patients with dementia and depression express behaviors such as biting, kicking, spitting or striking out in resistance to care combined with refusal of oral medications, what medication alternatives are available to improve quality of life?

METHOD: Mrs. T. was an 84-year-old woman who was treated on the Dementia Unit since 1/2013 and diagnosed with moderate to severe dementia with behavioral disturbance in addition to numerous comorbidities including coronary artery disease, diabetes mellitus type 2, and hypertension. Medications at the time of transfer to my care in 12/2013 were Zyprexa 2.5 mg po@ hs and a prn of Zyprexa 2.5 po for aggressive behaviors such as kicking, screaming and hitting staff. Her daughter was concerned as she had stopped eating, refused to get dressed and stopped taking all of her oral psychiatric and medical medications. She refused to open her eyes to speak with me on initial interview and hadn't eaten or bathed for several days. Depressive disorder not elsewhere classified was added and medication considered. In examining the evidence for an alternative depression medication, only selegiline (EMSAM) was supported by research evidence and available in transdermal administration and it had been underutilized because of dietary fears. Emsam $6 \mathrm{mg} /$ day was started as her diet would be controlled. There was no supportive evidence or trials for the use of topical administrations 
for the second generation antipsychotics. For agitated and aggressive behaviors, the pharmacist was consulted and Haldol $0.5 \mathrm{mg}$ topical gel bid was added. Quality of life behaviors targeted included eating meals, getting out of bed, dressing daily, and allowing a shower twice a week.

RESULTS: Three weeks following the start of EMSAM, Mrs. T. was sitting on the side of her bed eating breakfast. There were no side effects noted except for mild skin irritation that resolved. Her resistive behaviors continued especially at night care and the Haldol gel @ hs was increased $1 \mathrm{mg}$. By the 8th week of treatment, she was showering weekly, dressing most days, eating in the dining room, participating in group activities and motoring in her wheelchair. Positive results lasted 8 months, then symptoms returned. Mrs. T. had many cardiac events and 2 hospitalizations not related to psychiatric medications. She was maintained on the topical gels until her death from end-stage cardiac disease in $2 / 2015$.

CONCLUSIONS: There is a dearth of psychiatric research regarding transdermal medication administration. Since haldoperidol, olanzapine and risperidone can all be made into a transdermal application, research efforts should focus on randomized, placebo controlled design studies to assess their effectiveness in the patient with dementia with behaviors. EMSAM should be considered for depression when oral medication is refused or behaviors become vegetative.

\section{Can Naltrexone Induce Psychosis in Alcohol Dependence Patients with no Prior History of Psychosis?}

\section{Manoj Puthiyathu, MD'; Pankaj Manocha, MD' and Rumana Rahmani, $M D^{\prime}$}

${ }^{1}$ Bergen Regional Medical Center, Paramus, NJ

ABSTRACT: Introduction: Naltrexone is a competitive antagonist at all the opiate receptors (such as mu, delta and kappa), and has been FDA approved for treatment of alcohol dependence. Published literature, including randomized double blind placebo-controlled trails, has shown it to be well tolerated at $50 \mathrm{mg}$ with no serious adverse events. However, we report a case of a 43 -yearold Caucasian female who developed psychosis presenting as auditory hallucinations when started on naltrexone. The evidence to support this diagnosis is correlation between the timing of administration of naltrexone and onset of auditory hallucination, and the complete cessation of auditory hallucination following discontinuation of the medication. According to the algorithm of Naranjo et al., we assigned a score of 6 to this case indicating a probable adverse reaction to naltrexone.
EDUCATIONAL OBJECTIVE: In this review, the authors report an unusual case of naltrexone induced psychosis and propose pharmacological mechanism, which may cause such symptoms in patients with comorbid mental illness. Authors also study the therapeutic role of naltrexone in the management of alcohol dependence and the utility of appropriate dosages in such cases.

CONCLUSION: Psychiatrists should carefully monitor and assess for changes in symptom presentation to distinguish between the drug induced psychosis and a psychiatric illness. Reversal of symptoms on withdrawal of naltrexone can prevent unnecessary treatment with antipsychotics.

\section{A Patient Taking Lithium: The Impact of Air Travel and Dehydration}

Pankaj Manocha, $\mathrm{MD}^{1,2}$; Sanjeev Rally, $M D^{3}$ and John Gaston, $M D^{3,4}$

${ }^{1}$ Emory University School of Medicine, Atlanta, GA

${ }^{2}$ Bergen Regional Medical Center, Paramus, NJ

${ }^{3}$ Morehouse School of Medicine, Atlanta, GA

${ }^{4}$ Grady Memorial Hospital, Atlanta, GA

INTRODUCTION: Lithium is considered one of the most effective pharmacologic therapies for Bipolar Disorder. Studies indicate that approximately $75 \%$ of patients taking Lithium on a long-term basis are known to experience at least one episode of lithium toxicity. Lithium toxicity predominantly results from inappropriately high lithium doses or impaired lithium excretion. However, we present a case of a patient on regular scheduled lithium dose and a normal kidney function whose clinical impression is suggestive of lithium toxicity secondary to dehydration as a result of warm climate exposure and recent air travels.

CASE PRESENTATION: A 38 year old Caucasian male with a history of Bipolar Disorder (Type 1 with psychotic features) who is maintained on lithium therapy presented to the emergency department with disorganized thinking/confusion and red-tinged faced with periorbital sparing. The patient was unable to provide any history due to his mental status and was held involuntarily after he was found to have a lithium level of $1.8 \mathrm{mg} / \mathrm{dl}$. The patient's history is significant for multiple psychiatric hospitalizations. He has been maintained on lithium (900 mg TID) with his most recent blood level being $0.9 \mathrm{mg} / \mathrm{dl}$ (one month ago). The patient recently traveled by air through multiple states. In Minneapolis, his parents met with him and noticed that he appeared somewhat confused which they attributed to lack of sleep. While awaiting a connecting flight in Atlanta to his home in Portland, local authorities found him 
collapsed in the airport washroom. He was immediately rushed to our local hospital ER and was able to state that he needed help with lithium.

On initial presentation, he had signs of autonomic instability and was noted to be disorganized, tremulous, confused, and agitated with tachycardia. Significant admission labs included lithium level (1.8) and TSH (5.7). EKG showed sinus rhythm with evidence of T wave inversions and QTc prolongation $(499 \mathrm{msec})$ suggestive of lithium toxicity. Electrolytes, blood glucose level, WBC count, and CT head without contrast were within normal limits. Urine drug screen was negative, and alcohol level was $<10$. Lithium was discontinued, and the patient was started on intravenous fluids, thiamine, and folic acid. His lithium level gradually decreased to a therapeutic range, and he improved with supportive treatment including rehydration and correction of electrolyte imbalances.

CONCLUSIONS: This case illustrates that lithium toxicity can result from dehydration due to a combination of both warm climate exposure and air travel. The neurotoxic effects of lithium may induce delirium even in patients with normal kidney function. Psychiatrists should be aware of patient safety concerns when prescribing lithium to individuals with increased exposure to warm climates, UV sunlight, and the rigors of air travel, and should be encouraged to educate patients about proper usage.

\section{A Solitary Case of Treatment Resistant Depression: Where Do We Stand Now?}

Vandana Kethini, $M D^{\prime}$; Pankaj Manocha, $M D^{1,2}$ and Asghar Hossain, $M D^{\prime}$

${ }^{1}$ Bergen Regional Medical Center, Paramus, NJ

${ }^{2}$ Emory University School of Medicine, Atlanta, GA

INTRODUCTION: According to a WHO 2012 factsheet, depression affects 350 million worldwide and 18 million people at any one time in the US alone. Although there are known, effective treatments for depression, about $30-46 \%$ of patients with major depressive disorder (MDD) fail to fully respond to initial antidepressants. Incomplete remission of these depressive symptoms results in increased risk of relapse, decreased functioning in work and social settings, and increased risk of eventual suicide. The psychiatrist's approach to manage incomplete remission includes "being observant", interchanging antidepressants or adding another antidepressant, adjunctive treatment, cognitive behavioral therapy (CBT), trans magnetic stimulation (TMS), electroconvulsive therapy (ECT) or interpersonal psychotherapy (IPT). Several cases of treatment refractory depression (TRD) have been reported in literature so far.

CASE: We report a 40-year-old, single, Caucasian male with MDD since childhood who was unsuccessfully managed with several pharmacological and nonpharmacological modalities. Patient has been ineffectively tried on several antidepressants such as selective serotonin reuptake inhibitors (SSRIs), serotonin norepinephrine reuptake inhibitors (SNRIs), serotonin norepinephrine dopamine reuptake inhibitors (SNDRIs), tricyclic antidepressants (TCAs), tetracyclic antidepressants and monoamine oxidase inhibitors (MAOIs). Patient was also started on eszopiclone (hypnotics), lithium carbonate (mood stabilizer), aripiprazole (atypical antipsychotics), bupropion hydrochloride (amino ketone), ketamine (NMDA receptor antagonist) infusion, and other antidepressants such as lamotrigine (anticonvulsant) with minimal improvement. Aside from pharmacotherapy, several other treatments including TMS, ECT, CBT and IPT in past 2 years have shown minimal or no improvement in symptoms. To better assist patient with his depressive symptoms, patient started partial hospitalization, and was started on duloxetine (SNRI), vilazodone (combined SSRI and a 5-HT [1A] receptor partial agonist) and lisdexamfetamine (stimulant). Patient was compliant with the treatment and was recommended genecept assay to better understand his lack of response to several medications. The results showed that he has two allele's G/A for calcium channel. The A allele results in superfluous calcium channels, resulting in excessive calcium current. Based on the report patient was recently started on atypical antipsychotic (lurasidone) and will be followed up every 2-3 weeks in outpatient clinic at Bergen Regional.

EDUCATIONAL OBJECTIVE: Readers will appreciate and identify the pharmacological and non-pharmacological modalities used for managing TRD. Authors will also update medical professionals on newer models of care and management of TRD in current literature.

\section{Study of Decision Making Associated to Social Interactions in Depression Using an Interactive Behavioral Task}

Gabriela Fernández Theoduloz, BS ; Valentina Paz, $B S^{\prime}$; Eliana Nicolaisen, '; Alfonso Pérez,', Álvaro Cabana, $P h D^{\prime}$ and Victoria Gradin, $P h D^{\prime}$

${ }^{1}$ Center for Basic Research in Psychology. School of Psychology, Universidad de la República, Montevideo, Uruguay

ABSTRACT: Major depression is a prevalent disorder that significantly affects the social functioning of individuals. Depression is characterized by a negative cognitive style and socially challenging events could trigger dysfunctional negative thinking patterns in depressed people, leading to social avoidance and isolation. This highlights the importance of investigating 
decision making associated to social interactions in depression. In the last few years, tasks that recreate interactive scenarios have been used to provide a framework to operationalize social exchanges. In this study we developed an interactive behavioral task that allows the study of social avoidance preferences in depression. The study investigated the relation between depression and self-esteem, interpersonal problems and anxiety; and if depressed participants differ from controls in decision making and emotional responses in the behavioral task.

METHODS: Data were acquired from a group of participants meeting DSM-IV criteria for depression and a group of controls. Applicants were screened for depression and other psychiatric symptoms. Inclusion criteria for the depression group were satisfying DSM-IV criteria for an episode of depression plus a score $\geq 16$ on the Beck Depression Inventory and at least 3 weeks of not taking antidepressant medication. Participants in the control group had no current or past history of any psychiatric disorder. In the experimental session, participants were assessed for depression symptoms severity, anxiety, self-esteem and interpersonal problems. Participants were assessed with the behavioral task in which they have to choose between a social or an individual option. The social option allows participants to earn more points but it is socially challenging because the result depends on both players' performance. If one player fails they will not get the maximum score in the trial. In the individual option participants avoid the comparison with the other player and the responsibility of performing well.

RESULTS: Depression was associated with low self-esteem, anxiety and interpersonal problems, particularly in the domains of socially-inhibited and nonassertive. Participants in the depression group reported more anxiety knowing that they could meet their co-players at the end of the session. Also, we found that participants in the depression group preferred the individual option significantly more than controls.

CONCLUSIONS: The preference for playing individually among the depressed participants supports the idea that depressive people avoid stressful social situations, even when these could be profitable. These situations may trigger negative thoughts about the self that in turn amplify social avoidance. These findings may contribute to elucidate the cognitive and emotional processes that underlie social interactions in people with depression and may help to improve treatments that target social difficulties.

FUNDING: Academic Committee of Graduate Studies UdelaR
Improving Diagnostic Accuracy of Pediatric Bipolar Within a Private Practice

\section{Daniel Wesemann, LISW, MSN, PMHNP-BC, APRN}

Rush University, College of Nursing, Chicago, IL

INTRODUCTION: The diagnosis of pediatric bipolar disorder (PBD) is still controversial. Bipolar disorder is the sixth leading cause of disability worldwide and nearly $75 \%$ of adults diagnosed with bipolar disorder had symptoms prior to adulthood. Internationally PBD rates continue to grow and prevalence in the United States is estimated to be $1-2 \%$. A public health issue is differentiating those with PBD from those with ADHD. ADHD is the most common neurobiological disorder diagnosed in children; however those with PBD have more serious negative health outcomes. Providers continue to need measures to help differentiate these diagnoses.

METHOD: This is a Doctoral of Nursing Practice capstone project that looked at rates of PBD and ADHD in Iowa and locally at private practice clinic in Davenport, Iowa. A screening packet to differentiate between PBD and ADHD was developed using three valid and reliable tools: Child Mania Rating Scale, Child Behavior Checklist and the NICHQ Vanderbilt. Through convenience sampling, 102 screening packets were completed by parents of pediatric patients at GPA. These data were compared to provider's clinical diagnosis and patient's billing information. Symptom agreement analysis between the screeners and the provider diagnoses was performed using ANOVA and Tukey HSD post hoc analysis.

RESULTS: In Iowa from 2008 to 2013 there was a $33 \%$ decrease in PBD to $0.57 \%$ and at GPA there was a $51.2 \%$ decrease to $0.79 \%$. This contradicts increasing rates of $\mathrm{PBD}$ in the United States and internationally. Also rates of $\mathrm{ADHD}$ increased in the state of Iowa. The screening results showed at least $19.6 \%$ of the participants were positive for PBD. The screening results also indicated at least $21.78 \%$ were positive for ADHD. Comparing the screening data to the provider's clinical diagnosis there was no diagnostic agreement for PBD $(\mathrm{p}<0.05)$.

CONCLUSION: It is highly unlikely that nearly $20 \%$ of the patients at GPA have PBD. However these data do suggest that there is more than $0.79 \%$. Regionally this could be a trend to under diagnose PBD in favor of $\mathrm{ADHD}$ as reported by Iowa Medicaid billing information. Further studies are needed to replicate these data. Providers at GPA are now asked to use these screeners in their practice to further determine if the data obtained from the screening packets match their clinical judgments. 


\section{A 12-Month, Open-Label Study of The Safety and Tolerability of Lisdexamfetamine Dimesylate in Adults with Moderate to Severe Binge Eating Disorder}

Maria Gasior, $\mathrm{MD}, \mathrm{PhD}^{\prime}$; James Hudson, $\mathrm{MD}, \mathrm{Sc} \mathrm{D}^{2}$; Javier Quintero, $\mathrm{MD}^{3} ; \mathrm{M}$. Celeste Ferreira-Cornwell, PhD'; Jana Radewonuk, $M \mathrm{Sc}^{\prime}$ and Susan McElroy, $\mathrm{MD}^{4,5}$

${ }^{1}$ Shire Development LLC, Wayne, PA

${ }^{2}$ McLean Hospital/Harvard Medical School, Belmont, MA

${ }^{3}$ Hospital Universitario Infanta Leonor, Madrid, Spain

${ }^{4}$ Institution: Lindner Center of HOPE, Mason, OH

${ }^{5}$ University of Cincinnati College of Medicine, Cincinnati, $\mathrm{OH}$

STUDY OBJECTIVES: To assess the long-term safety and tolerability of lisdexamfetamine dimesylate (LDX) in adults with moderate to severe binge eating disorder (BED) in a phase 3 , multicenter, 12-month open-label extension study.

METHODS: Eligible adults (18-55 years) with BED who completed 1 of 3 antecedent trials were enrolled in a 52 week open-label extension study (4 weeks of dose optimization [initial dose, $30 \mathrm{mg}$ LDX; target doses, 50 or $70 \mathrm{mg} \mathrm{LDX];} 48$ weeks of dose maintenance). Safety evaluations included the occurrence of treatmentemergent adverse events (TEAEs), vital sign and weight assessments, and responses on the Columbia-Suicide Severity Rating Scale (C-SSRS).

RESULTS: 604 participants enrolled; 599 (521 females; 78 males) were included in the safety analysis set. Mean a SD LDX exposure was 284.3ą118.84 days; 344 participants $(57.4 \%)$ were treated for $\geq 12$ months. A total of $506(84.5 \%)$ participants reported TEAEs (TEAEs leading to discontinuation, 54 [9.0\%]; severe TEAEs, 42 [7.0\%]; serious TEAEs, 17 [2.8\%]). TEAEs reported in $\geq 10 \%$ of participants were dry mouth $(27.2 \%)$, headache $(13.2 \%)$, insomnia $(12.4 \%)$, and upper respiratory tract infection (11.4\%). Mean a SD changes from antecedent study baseline in systolic and diastolic blood pressure, pulse rate, and weight at week 52/early termination $(\mathrm{n}=597)$ were $2.19 \mathrm{a} 11.043$ and $1.77 \mathrm{a} 7.848 \mathrm{mmH}$, $6.58 \mathrm{a} 10.572 \mathrm{bpm}$, and $-7.04 \mathrm{a} 7.534 \mathrm{~kg}$, respectively. On the C-SSRS, there were 2 positive responses for any active suicidal ideations and 4 positive responses for nonsuicidal self-injurious behavior; there were no positive responses for suicidal behavior, actual suicide attempts, or completed suicides.

CONCLUSIONS: The long-term safety and tolerability of LDX was generally consistent with its known safety profile.

FUNDING: This clinical research was sponsored by Shire Development LLC.
PREVIOUS PRESENTATIONS: These findings have been presented at the 21st Annual Meeting of the Eating Disorders Research Society (Taormina, Sicily, Italy; September 17-19, 2015).

\section{Helium Assisted Suicide a Serious Public Health Concern: A Rare Case Report of 20-Year-0ld Male with Helium Induced Suicide Attempt}

\section{Deepti Kaul Mughal, MD}

TMS of Jacksonville, Jacksonville, FL

ABSTRACT: Suicide is the deliberate act of killing oneself. A total of 842,000 people in the world died from suicide in 2013. It has become a major public health problem, and 10th leading cause of death worldwide. Suicide by helium inhalation has become popular in recent years. It has been cited in right-to-die literature. Internet suicide forums also depict it as an effective, painless, and peaceful method of suicide. Despite its lack of direct toxicity, helium's physical properties make it a very dangerous substance. Inhaled helium quickly displaces other gases in air, most importantly oxygen and carbon dioxide. Breathing reflex in humans is not triggered by the lack of oxygen, but rather by the excess of carbon dioxide. Inhaled helium displaces carbon dioxide, resulting in abolition of apneic reflex. When pure helium is inhaled, unconsciousness occurs immediately and death occurs within minutes. The purpose of this case report is to analyze the process of assisted suicide, with helium, and also to create awareness among medical society, and regulatory bodies to prevent availability of another lethal but convenient way of suicide.

CASE REPORT: A 20-year-old Caucasian male was admitted in our psychiatric unit for an attempted suicide with the use of a helium tank and mask. Patient had no history of any other psychiatric disorder or substance use. Six months ago patient was rejected from the Navy because of mild cerebral palsy. Patient felt very hopeless and depressed and contemplated suicide at that time. Three months ago, he began researching methods of suicide on the Internet. He decided to use a helium tank and mask, because this method apparently seemed to him an easy way to attempt suicide. He rented the helium tank from the local balloon shop and ordered the mask on Internet.

DISCUSSION: Helium gas is freely available in refillable tanks, for filling balloons. This easy availability has contributed to increasing incidence of suicidal attempts with helium. Asphyxial suicide by using helium is also practiced by use of plastic bag over the head. There are very few, published cases of suicide by helium, worldwide. Another major problem is that helium inhalation 
can be easily disguised, as a method of assisted suicide. Helium cannot be detected using standard toxicological analysis of blood and urine samples. Special autopsy techniques are needed for confirmation. The public and clinicians, in particular psychiatrists and primary care physicians, need to be aware of helium inhalation as a highly lethal method of assisted suicide. Pathological and laboratory tests to detect the helium in the body should be readily available in toxicology laboratories. It is necessary that regulatory bodies should mandate reporting of suicide by helium to health departments and a national database be maintained. There is dire need for better regulations from the governing bodies to prevent the introduction of this novel method of committing suicide.

\section{Trisomy $\mathrm{X}$ and Psychosis}

\author{
Sheema Imran, $\mathrm{MD}^{\prime}$; Ashley Pearl Mehlman ${ }^{2}$ and \\ Samana Zaidi ${ }^{2}$ \\ ${ }^{1}$ Bergen Regional Medical Center, Paramus, NJ \\ ${ }^{2}$ St. George's University School of Medicine, True \\ Blue, Grenada
}

ABSTRACT: Triple $\mathrm{X}$ syndrome affects up to 1 in 1,000 female births. The clinical presentation varies from asymptomatic to developmental delay, physical abnormalities and abnormal social behavior. This case report presents a patient with triple $\mathrm{X}$ syndrome who, on the spectrum of clinical features, exhibited signs of moderate disease. Her most recent psychiatric evaluation was conducted at Bergen Regional Medical Center, where she was found to be psychotic with a history of pervasive developmental disorder and posttraumatic stress disorder. This case displays the importance of defining such patients within the context of social stressors, especially from peers who reject them based on intellectual deficits and physical appearance. With this in mind, a possible association between patients with triple $\mathrm{X}$ syndrome and psychosis is explored.

\section{Comparing Deep Brain Stimulation (DBS) and Ect for Treatment Resistant Depression in Elderly Population}

Laima Spokas, $M D^{\prime}$; Vandana Doda, $M D^{\prime}$; Rumana Rahmani, $M D^{\prime}$ and Asghar Hossain, $M D^{\prime}$

\footnotetext{
${ }^{1}$ Bergen Regional Medical Center, Paramus, NJ
}

INTRODUCTION: Depression in the elderly population is a major public health problem worldwide. Geriatric depression is associated with poor prognosis. The pharmacological use in this population is limited due to reduced tolerability, increased risk of drug interactions and non-compliance associated with cognitive decline.
Elderly depression not only increases mortality and morbidity in chronic diseases but also increases disability, health care burden and cost. ECT has been a safe treatment option for treatment resistant elderly depression. Neuromodulation therapies have also been researched in the last decade. Deep brain stimulation, in which specific area of the brain are stimulated by implanting electrodes, is a newer experimental treatment option that can be considered for treatment resistant depression among elderly population in the future.

OBJECTIVE: To compare and contrast ECT with deep brain stimulation for treatment resistant depression in elderly. We will also discuss its technique and compare indications, side effects and efficacy.

METHOD: The literature search was done by using PubMed on deep brain stimulation (DBS) and ECT for treatment resistant depression in elderly, published between 20002015.

RESULTS: We found that ECT is safer and more effective treatment modality for geriatric depression as compared to deep brain stimulation (DBS). Preliminary studies have shown that newer ECT technologies such as Ultrabrief RUL (right unilateral) is equally effective as BF (bifrontal) pulse technology and both produce less cognitive side effects as compared to regular ECT. Although DBS is the most invasive among all neuromodulation therapies, it seems to offer positive results in preliminary studies in treatment resistant major depression. Studies have shown decreased depressive symptoms and decreased relapse rates in patients with treatment resistant major depression. Currently, different brain areas are being investigated. DBS is a well-known treatment modality for elderly patients suffering from treatment resistant Parkinson's disease and other movement disorders but it is still an experimental treatment modality for treatment resistant elderly depression and more data and studies involving large sample size and elderly population are needed to confirm the safety and efficacy of DBS in elderly depression.

CONCLUSION: ECT remains the most effective treatment modality for treatment resistant depression in the elderly population. Deep brain stimulation lacks specific studies in the elderly depression. More studies and data involving geriatric population are needed.

\section{Efficacy of Acute Treatment with Aripiprazole Once-Monthly on Marder Factor Scores in Patients with Schizophrenia}

John M. Kane, MD ${ }^{1,2}$; Maia Miguelez, PhD ${ }^{3}$; Timothy Peters-Strickland, $\mathrm{MD}^{4}$; $\mathrm{Na}$ Jin, $\mathrm{MS}^{5}$; Pamela Perry, $\mathrm{MS}^{4}$; Peter Hertel, PhD ${ }^{6}$; Christine Elizabeth Farkas, PharmD, BCPP ; Anna Eramo, $\mathrm{MD}^{7}$; Raymond 
Sanchez, $M D^{4}$; Robert McQuade, $P h D^{8}$ and Zahinoor Ismail, $M D, F R C P C^{9}$

${ }^{1}$ Zucker Hillside Hospital, Glen Oaks, NY

${ }^{2}$ Hofstra North Shore-LIJ School of Medicine, Glen

Oaks, NY

${ }^{3}$ Otsuka Canada Pharmaceutical, Inc., Saint-Laurent, Quebec, Canada

${ }^{4}$ Otsuka Pharmaceutical Development \&

Commercialization, Inc., Princeton, NJ

${ }^{5}$ Otsuka Pharmaceutical Development \&

Commercialization, Inc., Rockville, MD

${ }^{6}$ H. Lundbeck A/S, Valby, Denmark

${ }^{7}$ Lundbeck LLC, Deerfield, IL

${ }^{8}$ Otsuka America Pharmaceutical Inc., Princeton, NJ

${ }^{9}$ Hotchkiss Brain Institute, University of Calgary,

Alberta, Canada

OBJECTIVE: Aripiprazole once-monthly $400 \mathrm{mg}$ (AOM 400) demonstrated significant improvements in symptoms and functioning versus placebo, and good tolerability in patients with an acute exacerbation of schizophrenia (1). The 5 Marder factors of the Positive and Negative Syndrome Scale (PANSS) are well validated and widely used to provide a complete representation of the symptom range in schizophrenia (2). This post hoc analysis investigates effects of AOM 400 on a broad range of symptoms in the acute treatment of schizophrenia using the 5 PANSS Marder factors.

METHODS: In a randomized, double-blind, placebo-controlled, 12-week study, AOM 400 was compared with placebo in adults with schizophrenia experiencing an acute psychotic episode. The clinician-rated PANSS scale comprises 30 items rating symptoms from 1 (not present) to 7 (extremely severe). PANSS item scores are grouped by Marder factors: positive symptoms (8 items), negative symptoms (7 items), disorganized thought (7 items), uncontrolled hostility/excitement (4 items), depression/anxiety symptoms (4 items), and in the PANSS excited component (PEC, 5 items). Changes from baseline to week 12 in PANSS Marder factors were assessed using a mixed model of repeated measures with fixed effects of treatment, region, week, and treatmentby-week interaction, and baseline-by-week interaction as covariates.

RESULTS: Significant improvements in Marder factor scores with AOM $400(\mathrm{n}=162)$ vs placebo $(\mathrm{n}=167)$ were demonstrated by least squares mean changes $[95 \%$ $\mathrm{CI}]$ from baseline to week 12 of $-4.5[-6.0,-3.0]$ $(\mathrm{P}<0.0001)$ for positive symptoms, $-2.5[-3.8,-1.3]$ $(P=0.0001)$ for negative symptoms, $-3.4[-4.7,-2.1]$ $(P<0.0001)$ for disorganized thought, $-2.4[-3.3,-1.5]$ $(P<0.0001)$ for uncontrolled hostility/excitement, -1.7 [-2.5, -0.8] $(P=0.0002)$ for anxiety/depression, and -2.8 $[-3.9,-1.8](P<0.0001)$ for PEC. At baseline,
PANSS Marder factor mean scores $( \pm$ SD) were similar between treatment groups: positive symptoms (AOM 400: $33.1 \pm 3.7$; placebo: $33.1 \pm 3.4)$, negative symptoms (AOM 400: $22.9 \pm 5.1$; placebo: $23.8 \pm 4.5)$, disorganized thought (AOM 400: 24.1 \pm 3.7 ; placebo: $24.4 \pm 3.9$ ), uncontrolled hostility/excitement (AOM 400: $10.0 \pm 3.5$; placebo: $9.9 \pm 3.2$ ), anxiety/depression (AOM 400: 12.2 \pm 2.9 ; placebo: $12.2 \pm 2.8$ ), and PEC (AOM 400: $13.2 \pm 3.9$; placebo: $13.2 \pm 3.6$ ). The improvements with AOM 400 vs placebo were significant from week 1-2 on all Marder factors and remained significant through to endpoint.

CONCLUSIONS: In acutely ill patients with schizophrenia, treatment with AOM 400 was efficacious and demonstrated fast and sustained onset of action across all domains of the illness conceptualized by PANSS Marder factors.

FUNDING: This study was funded by Otsuka Pharmaceutical Development \& Commercialization, Inc.

\section{REFERENCES}

1. Kane JM, Peters-Strickland T, Baker RA, et al. J Clin Psychiatry. 2014; 75(11): 1254-1260.

2. Marder SR, Davis JM, Chouinard G. J Clin Psychiatry. 1997; 58(12): $538-546$

Collaboration of Psychiatry and Applied Behavior Analysis: An Interdisciplinary Approach to Reducing Polypharmacy and Treating Challenging Behaviors

Anna Marie DiPietro, CRNP'; Elbeth Dayton, BCBA, Jennifer Quigley, BCBA; Timothy Nipe, BCBA and Jim Chok, BCBA-D

${ }^{1}$ Melmark, INC, Berwyn, PA

ABSTRACT: In clinical practice, psychiatric practitioners and behavior analysts often make changes to an individuals' medication and behavioral treatment package without much collaboration between the two disciplines. Integrated efforts in the fields of psychiatry and behavior analysis are spare in both research literatures as well as in applied settings. The Residential Treatment Facility (RTF) at Melmark specializes in the assessment and treatment of severe and treatment resistant challenging behavior for children with intellectual disability and co-morbid psychiatric/neurological disorders. The RTF provides intensive clinical services, using applied behavior analysis (ABA), as well as comprehensive psychiatric and rehabilitative care within a short-term residential placement. Data collection occurs 24 hours a day and is reviewed on an ongoing basis by a multidisciplinary team representing the fields of behavior analysis, psychiatry, psychology, and healthcare. This poster will present retrospective medication and 
behavioral data from 39 individuals. Systematic manipulation of medication packages and dosage changes with frequent review and collaboration between clinicians and psychiatric practitioners, in conjunction with intensive behavioral intervention, led to a decrease in both challenging behavior as well as polypharmacy. This poster serves to illustrate the importance of collaboration between psychiatric professionals and board certified behavior analysts (BCBA) in the treatment of severe challenging behavior in individuals with autism spectrum disorders and intellectual disabilities.

\section{Effects of Hydroxyzine on Cognition}

Suhey Gissell Franco Cadet, $M D^{\prime}$; Imran Quereshi, $M D^{\prime}$; Asgar Hossain, $\mathrm{MD}^{\prime}$ and Amandeep Kaur Bhinder

${ }^{1}$ Bergen Regional Medical Center, Paramus, NJ

ABSTRACT: Brain histamine is involved in a wide range of physiological functions such as regulation of the sleep-wake cycle, arousal, cognition and memory, mainly through H1 receptors. Histamine antagonists, often prescribed for allergic disorders, sometimes can induce sleepiness and cognitive deficits. It is essential that antihistamines maintain the integrity of the cognitive system. First-generation antihistamines (Hydroxyzine)) are effective $H$ (1)-receptor antagonists; they are also highly lipophilic and readily cross the blood-brain barrier. Subjects who were treated with the first-generation were found to have significant performance deficits on tests of divided attention, working memory, vigilance, and speed. The sedative effects of the Vistaril persist well into the next day and thus can potentially interfere with daytime performance and safety even when taken the night before. It is therefore recommended that patients whose occupations require vigilance, divided attention, or concentration receive it with caution. It was concluded that histamine and $\mathrm{H} 1$ receptors are involved in maintaining arousal and cognition, and the severity of clinical symptoms is correlated with the amount of antihistamine that penetrate into the brain.

The goal of this literature review is to investigate the effects of Hydroxyzine (Vistaril) on cognition, with the objective to optimize patient response to treatment and to minimize side effects, including cognition decline. Comparison of timing of the dosage, side effect profile and effects on cognition with other drugs will be discussed.

\section{Encenicline Increases the Matrics Consensus Cognitive Battery (MCCB) Neurocognitive Composite Score in Patients with Schizophrenia}

Andrew P. Ho, MD'; Keith Nuechterlein; Richard Keefe; Nancy Dgetluck; Vicki Davis; Ji Yu; and Dana Hilt

${ }^{1}$ Forum Pharma
BACKGROUND: Patients with schizophrenia suffer from broad and significant cognitive impairment and perform $\sim 1$ to 1.5 standard deviations below normal controls. This degree of impairment is analogous to 15 IQ points. Encenicline is an alpha7 nicotinic acetylcholine receptor agonist and may improve cognition in patients with schizophrenia. U.S. subjects with schizophrenia in the encenicline Phase 2B study were assessed with the MCCB, a 10-test battery assessing 7 domains of cognition. Because social cognition is distinct from neurocognition, the MCCB scoring software was updated in 2015 to support a new MCCB Neurocognitive Composite score, calculated without the social cognition domain [MSCEIT]. The new software made possible a new posthoc analysis.

METHODS: Patients with schizophrenia on a stable dose of atypical antipsychotics were randomized to encenicline $0.27,0.9 \mathrm{mg}$, or placebo once daily for 12 weeks. Qualified raters assessed subjects with the MCCB at baseline, Day 44, and Day 84. To mirror the population of the ongoing Phase 3 studies, subjects with age $<50$ were included in this post-hoc analysis $(\mathrm{N}=104)$.

RESULTS: Encenicline $0.27 \mathrm{mg}$ and $0.9 \mathrm{mg}$ were statistically superior to placebo at Day 84 as measured by the MCCB Neurocognitive Composite Score $(p=0.038$, $\mathrm{p}=0.024$ respectively). Effect size of the $0.27 \mathrm{mg}$ and $0.9 \mathrm{mg}$ group was 0.55 and $0.57(\mathrm{NNT} \sim 4)$. There were no significant clinical or laboratory safety/tolerability findings.

CONCLUSIONS: Re-analysis of the Encenicline Phase 2B data using the recently published MCCB Neurocognitive Composite Score supports further development of Encenicline for cognitive impairment of schizophrenia. Confirmatory Phase 3 studies are currently on-going.

\section{Refractory Psychosis in a Patient with Mega Cisterna Magna}

Narissa R. Etwaroo, $\mathrm{MD}^{\prime}$; Astik Joshi, $M D^{\prime}$; Rita Yanez Horton, $M D^{\prime}$ and Donard Dwyer, $P h D^{\prime}$

${ }^{1}$ Louisiana State University, Lafayette, LA

ABSTRACT: Summary: This is a case of a Hispanic female with a past psychiatric history of treatment resistant psychosis and prior suicidal attempt that presented to the hospital with auditory hallucinations and suicidal ideations. Throughout hospitalization, patient continued to be psychotic in spite of treatment with several typical and atypical antipsychotics, antidepressants and inpatient milieu therapy. Interestingly, while her symptoms did not ameliorate in response to medications, she did develop side effects associated with the drugs given, including 
lipid derangement, weight gain and development of dyskinesia. Analysis of serum levels olanzapine showed a supra therapeutic level. This concluded that her ongoing psychosis was not due to noncompliance with treatment or ineffective levels of medication. On further workup for treatment resistant psychosis she was found to have Mega Cisterna Magna on her MRI Brain revealing possible association between neuroanatomical defects and refractory psychosis. This patient was also found to have vitamin $\mathrm{D}$ insufficiency which is hypothesized to be neuro-protective and may play an additive role in her deficit and treatment refractoriness.

Mega Cisterna Magna refers to significantly enlarged CSF retro-cerebellar cisterns in the posterior fossa with normal cerebellar morphology. It is a variant of DandyWalker Malformation which needs to be further studied to determine if it is a normal variant or has clinical significance in neurological as well as psychiatric illness.

DISCUSSION: To our knowledge, this is the only case report that links refractory psychosis with Mega Cisterna Magna and vitamin D insufficiency. Mega Cisterna Magna has previously been associated with schizophrenia and bipolar disorder. This brain abnormality may contribute to disturbances in the cortico-cerebellarthalamic-cortical pathway associated with cognitive dysmetria. The neuroanatomical defect, mega cisterna magna may explain the cognitive dysfunction observed in our patient and provided an organic basis for her lack of response to treatment. Patient was also found to have vitamin $\mathrm{D}$ insufficiency which could exacerbate degenerative or functional decline in the brain due to a decrease in its neuro-protective properties. Neuroimaging and vitamin $\mathrm{D}$ levels need to be looked at as they could account for the treatment refractoriness.

\section{Reduced B Vitamin Therapy for MDD in MTHFR C677t/A1298c Patients - Clinical Response Correlates with Homocysteine Reduction: A Double-Blind, Placebo-Controlled Study}

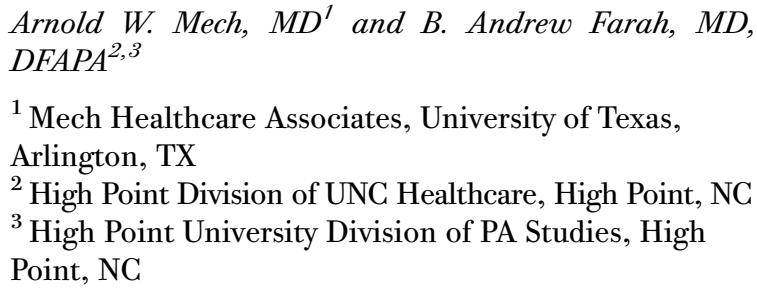

${ }^{1}$ Mech Healthcare Associates, University of Texas, Arlington, TX

${ }^{2}$ High Point Division of UNC Healthcare, High Point, NC

${ }^{3}$ High Point University Division of PA Studies, High Point, NC

ABSTRACT: This study was designed to evaluate the efficacy and safety of reduced B vitamins as monotherapy in adults with major depressive disorder (MDD) who were also positive for at least one of the common MTHFR polymorphisms associated with depression: C677T and/or A1298C. The study further tested the hypothesis that reduced (metabolized) B vitamins will reliably lower plasma homocysteine (HCY), and therefore CNS homocysteine. These findings have implications beyond MDD, as elevated HCY levels are not only associated with depressive disorders, including postpartum depression, but numerous other illnesses and conditions, such as dementias and stroke.

A total of 330 adult patients with MTHFR C677T and/or A1298C polymorphism who met the Diagnostic and Statistical Manual V criteria for MDD without psychosis were enrolled in the trial. 160 were randomized to receive placebo, while 170 received a gel capsule containing a combination of reduced B vitamins (B1, B2, B6, B12, and three forms of B9) and micronutrients designed to lower HCY in the CNS. Specifically, the active ingredients included citrated folic acid, folinic acid (leucovorin), l-methylfolate magnesium, thiamine pyrophosphate, flavin adenine dinucleotide, pyridoxal 5 - phosphate, and adenosylcobalamin, other $b$ vitamin coenzymes, mineral cofactors, betaine, and omega 3s. MTHFR status was determined initially, and plasma HCY levels were drawn at baseline and at eight weeks for all subjects. Subjects were screened for potential side effects and adverse events at weeks two, four, and eight. The MADRS was used to determine efficacy for MDD.

All subjects met the criteria for MDD and were positive for the MTHFR polymorphisms associated with depressive disorders. While 159 of $170 \mathrm{~B}$ vitamin patients completed the 8-week study, 123 of the 160 patients receiving placebo were full completers. Of the 159 patient completers who received reduced B's, 131 (82.4\%) showed a reduction in plasma homocysteine (an average of $2.7 \mu \mathrm{mol} / \mathrm{L}$ from a baseline of $9.6 \mu \mathrm{mol} / \mathrm{L}$, $p<0.001)$, while $28(17.6 \%)$ showed no significant change in plasma HCY. The 123 patients who received placebo demonstrated a small elevation in HCY levels (of $0.4 \mu \mathrm{mol} / \mathrm{L})$.

Active treatment patients demonstrated, on average, a 12 point reduction on the MADRS by week 8 , and $42 \%$ of vitamin monotherapy patients had achieved full remission by the end of the trial $(p<0.001)$. No side effect was significantly different between placebo and active treatment groups. No patients experienced mania in either group.

A combination of reduced $B$ vitamins and micronutrients in capsule form, indicated for conditions associated with lower than optimal folate levels in the CNS and resulting hyperhomocyteinemia, when used in the treatment of MDD in MTHFR positive patients, significantly separated from placebo by week two of treatment on the MADRS rating scale, and separation expanded throughout the trial. Baseline Montgomery-Asberg score was reduced by 12 points on average in the treatment group with 55 of $131(42 \%)$ achieving remission by the week eight visit. Further, treatment response was associated with a significant reduction in HCY levels, 
$32.9 \%$ compared to placebo. Patients receiving placebo experienced, on average, a slight, non-significant increase in HCY levels of $0.4 \mu \mathrm{mol} / \mathrm{L}$. These results confirm the HCY theory of depression and the therapeutic benefit and safety of using reduced $B$ vitamins as monotherapy in depressive disorders particularly in the presence of MTHFR polymorphisms.

\section{Mapping of Intoxications and Suicide Attempts of Children and Adolescents in a Southern State of Brazil}

Rosiane G. M. Zibetti, PhD ${ }^{1,2}$; Karyna Turra, MS ${ }^{\prime}$; Mary Anne P. Smarczewski, $M S^{3}$ and Roberto Rosati, $P h D^{1,2}$

${ }^{1}$ Pequeno Príncipe College, Curitiba, PR, Brazil

${ }^{2}$ Pelé Pequeno Príncipe Research Institute, Curitiba, PR, Brazil

${ }^{3}$ UNIOESTE, Cascavel, PR, Brazil

OBJECTIVES: Poisoning of children and adolescents is considered one of the most significant causes of morbidity and mortality, and it has been continuously increasing. Most poisonings occur at home and are due to pharmaceutic products or chemical. Faced with this reality, the aim of the current study was to know the profile of children and adolescent poisonings reported from 2007 to 2011 in the southern state of Brazil, with emphasis on cases of suicide.

METHODS: This is an exploratory-descriptive study with quantitative approach developed through the retrospective documentary analysis method. Information was collected in the surveillance database on Health Secretariat of the State of Paraná (southern state of Brazil), and analyzed the following variables: gender, age, circumstances of exposure contamination, toxic agent group, general classification and toxic agent. The data were plotted in a web mapping system geology system for medical research called Geomedicine (Ibanez, 2007).

RESULTS: 12,495 notifications were found, of which 3,506 were recorded as suicide attempt; the dominant age group is $16-19$ years, with $85.3 \%$, followed by $12-15$ years, with $84 \%$. The toxic agents used were pesticides with $14.5 \%, 13 \%$ anxiolytics, antidepressants with $11.3 \%$, $6 \%$ anticonvulsants, household cleaning $3.8 \%$, and analgesics and NSAIDs 5\%. Of the total suicide attempt notifications, $0.9 \%$ (33) evolved to death records.

CONCLUSIONS: Suicides result from a complex interaction of biological, genetic, psychological, sociological, cultural and environmental factors. A form of prevention would be early detection in the community of suicidal behavior or suicidal ideation, and referral to specialists. This mapping is important to show the incidence and location of cases to public health actions.
Deuterium-Modified Dextromethorphan and Ultra Low-Dose Quinidine (AVP-786; D6 DM/Q): Phase I Drug Interaction Trial with Paroxetine And Duloxetine

Nadine Knowles ${ }^{\prime}$; Laura Pope, PhD' and Joao Siffert, $M D^{\prime}$

${ }^{1}$ Avanir Pharmaceuticals, Inc., Aliso Viejo, CA

OBJECTIVES: AVP-786 (d6-DM/Q) is a combination of deuterated dextromethorphan (d6-DM) and ultra-low dose quinidine $(\mathrm{Q})$ being studied adjunctively in major depressive disorder. Deuterium, a non-radioactive isotope of hydrogen, is ubiquitous in the environment; the average adult body contains 2 grams of deuterium. Deuteration of DM significantly reduces CYP2D6 metabolism of d6-DM. Combining d6-DM with quinidine (Q), a CYP2D6 inhibitor, increases d6-DM bioavailability. This phase 1 study evaluated pharmacokinetic (PK) interactions between AVP-786 (d6-DM $30 \mathrm{mg}$ and Q $4.75 \mathrm{mg}$ BID), and the CYP2D6 substrates/inhibitors paroxetine (PRX; 20 $\mathrm{mg} / \mathrm{d}$ ) or duloxetine (DUL; $20 \mathrm{mg}$ BID).

METHODS: Single-center, open-label, sequential drug interaction study in healthy volunteers (aged 18-50 years, BMI $18-30 \mathrm{~kg} / \mathrm{m}^{2}$, genotyped as extensive metabolizers of CYP2D6). Subjects enrolled in 1 of 4 groups ( $\mathrm{N}=14$ each): Group 1, PRX days 1-20, AVP-786 days 13-20; Group 2, AVP-786 days 1-20, PRX days 9-20; Group 3, DUL days 1-13, AVP-786 days 6-13; Group 4, AVP-786 days 1-13, DUL days 9-13. Pharmacokinetic parameters $\left(\mathrm{AUC}, \mathrm{C}_{\max }, \mathrm{T}_{\max }, \mathrm{t}_{1 / 2}, \mathrm{C}_{\min }\right.$ and $\mathrm{T}_{\text {min }}$ ) were derived using non-compartmental methods for relevant analytes (d6-DM, d3-dextrorphan (d3-DX), $\mathrm{Q}$, and PRX or DUL). Single vs combined administrations were compared in each group using geometric mean ratios of $\mathrm{C}_{\max }$ and $\mathrm{AUC}_{0-12}\left(\mathrm{AUC}_{0-24}\right.$ for paroxetine) with $90 \%$ confidence intervals. Safety and tolerability were assessed.

RESULTS: Of 56 subjects enrolled, 50 completed $(\mathrm{n}=11$, 14,14 , and 11 in Groups 1, 2, 3, and 4, respectively). In Group 1, AVP-786 coadministration increased steadystate AUC of PRX 1.64 fold and $\mathrm{C}_{\max } 1.49$ fold vs PRX alone. In Group 2, PRX coadministration increased AUC and $\mathrm{C}_{\max }$ of AVP-786 components d6-DM (2.18 fold and 1.81 fold) and Q (1.33 fold and 1.25 fold) with a decrease in exposure to the d3-DX metabolite (AUC decrease 28.4\%; $\mathrm{C}_{\max }$ decrease 22.9\%) compared with AVP-786 alone. In Group 3, AVP-786 coadministration increased steady-state AUC of DUL 1.84 fold and $\mathrm{C}_{\max } 1.68$ fold vs DUL alone. In Group 4, DUL did not appreciably affect AVP-786 components (d6-DM or Q), nor the d3-DX metabolite. Monotherapy vs combination therapy AE percentages were: Group 1: $50 \%$ vs. 83\%; Group 2: $64 \%$ vs. 93\%; Group 3: $43 \%$ vs. 50\%; and Group 4: $50 \%$ vs. 
45\%. The most common AEs were dizziness, diarrhea, headache in Groups 1 and 2 (AVP-786 and PRX) and dizziness, headache, fatigue and muscle tightness in Groups 3 and 4 (AVP-786 and DUL). Clinical laboratories, vital signs, and ECG assessments were unremarkable between treatments.

CONCLUSIONS: In this phase I study, AVP-786 coadministration increased AUC to PRX ( 1.6 fold) and DUL ( $~ 1.8$ fold) vs either drug alone. Likewise, PRX increased the d6-DM AUC $\sim 2.2$ fold with a corresponding decrease $(\sim 28 \%)$ in $\mathrm{d} 3$-DX metabolite exposure; $\mathrm{Q}$ exposure was modestly increased $(\sim 33 \%)$. DUL did not appear to affect exposure to d6-DM, its metabolite or Q.

SUPPORTED BY: Avanir Pharmaceuticals, Inc.

\section{Efficacy, Safety, and Tolerability of Vortioxetine for the Treatment of Major Depressive Disorder in Patients Aged 55 Years or Older}

George Nomikos, MD, PhD'; Dapo Tomori, MD'; Wei Zhong, $\mathrm{PhD}^{\prime}$ and William Palo, $\mathrm{MS}^{\prime}$

${ }^{1}$ Takeda Development Center Americas, Deerfield, IL

ABSTRACT: Study Objective: Major depressive disorder (MDD) is the most prevalent psychiatric disorder in older adults $(9-18 \%$ of individuals aged $\geq 55 y)$ and increases mortality risk by 2-3 times than that of nondepressed older individuals (Tedeschini et al., 2011). This post hoc exploratory analysis evaluated the efficacy, safety, and tolerability of vortioxetine $5-20 \mathrm{mg} / \mathrm{d}$ in MDD patients aged $\geq 55 y$.

METHOD: Study-level efficacy data from 12 short-term (6-8wks), placebo (PBO)-controlled trials of vortioxetine $5-20 \mathrm{mg} / \mathrm{d}$ in adult MDD patients were assessed (full analysis set), and a random-effects meta-analysis was conducted with additional tests for heterogeneity. Safety and tolerability data were pooled from the same group of studies (all patients treated set). Patients met the DSMIV criteria for a major depressive episode and had a baseline Montgomery-Åsberg Depression Rating Scale (MADRS) score ranging from 22-30. Efficacy outcomes included the difference from $\mathrm{PBO}$ in change from baseline (CFB) to study endpoint in MADRS total score (MMRM) and response and remission (LOCF). Safety and tolerability assessment was based on the nature, incidence, and severity of adverse events (AEs) and vital signs during treatment.

RESULTS: A total of 1508 patients aged $\geq 55 \mathrm{y}$ were treated in the 12 studies (PBO $\mathrm{n}=561$; vortioxetine $5-20 \mathrm{mg}$ $\mathrm{n}=947$ ). Patients were mostly female (PBO 64.0\%; vortioxetine $67.1 \%$ ) and Caucasian $(92.0 \%$; 90.1\%). Concomitant medications with an incidence $\geq 10 \%$ in any treatment group included ACE inhibitors, lipidmodifying agents, analgesics, anti-inflammatory and anti-rheumatic products, vitamins, beta-blocking agents, drugs for acid-related disorders, and thyroid therapy. The mean difference from $\mathrm{PBO}$ in CFB in MADRS was -2.56 (5mg $\quad \mathrm{n}=324 \quad \mathrm{P}=0.035), \quad-2.87 \quad(10 \mathrm{mg} \quad \mathrm{n}=222$ $\mathrm{P}=0.007),-1.32 \quad(15 \mathrm{mg} \mathrm{n}=90 \mathrm{P}=\mathrm{NS})$, and -4.65 (20mg $\mathrm{n}=165 \mathrm{P}=0.012$ ). Odds ratios (ORs) for response vs $\mathrm{PBO}$ were 1.6 (5mg $\mathrm{P}=\mathrm{NS}), 1.8(10 \mathrm{mg}$ $\mathrm{P}=0.002), \quad 1.2 \quad(15 \mathrm{mg} \quad \mathrm{P}=\mathrm{NS}), \quad$ and $2.5 \quad(20 \mathrm{mg}$ $\mathrm{P}<0.001)$. ORs for remission vs $\mathrm{PBO}$ were $1.4(5 \mathrm{mg}$ $\mathrm{P}=\mathrm{NS}), 1.4$ (10mg $\mathrm{P}=\mathrm{NS}), 1.4(15 \mathrm{mg} \mathrm{P}=\mathrm{NS})$, and 2.7 (20mg $\mathrm{P}<0.001$ ). The incidence of patients with treatment-emergent AEs (TEAEs) was $61.5 \%$ for PBO and did not increase with higher vortioxetine doses $(5 \mathrm{mg}$ $63.0 \%$; 10mg 56.4\%; 15mg 67.8\%; 20mg 65.5\%). The incidence of TEAEs leading to discontinuation was higher than $\mathrm{PBO}$ at higher vortioxetine doses (PBO $5.2 \%$; $5 \mathrm{mg} 5.1 \%$; 10mg 5.0\%; 15mg $12.7 \%$; $20 \mathrm{mg} 9.8 \%$ ). The incidence of serious AEs was low with no increase with higher vortioxetine doses (PBO 1.2\%; $5 \mathrm{mg} 2.1 \%$; $10 \mathrm{mg} 0.8 \%$; $15 \mathrm{mg} 0.8 \%$; $20 \mathrm{mg} 0 \%$ ). The most common TEAEs (incidence $\geq 10 \%$ in any treatment group) were nausea, headache, and diarrhea. No clinically significant changes in vital signs emerged during treatment.

CONCLUSIONS: Vortioxetine was efficacious and well tolerated at available doses of 5,10 , and $20 \mathrm{mg}$ in MDD patients aged $\geq 55 \mathrm{y}$ with comorbid conditions and/or treated with concomitant medications.

FUNDING: Takeda Pharmaceutical Company, Ltd. and H. Lundbeck A/S.

\section{Developing a Team Model in Psychiatry: Incorporating an Emphasis on Exercise}

\author{
Richard Wallis, MSN, APRN, PMHNP ${ }^{1,2}$ and Mélinda \\ McCusker, MSN, APRN, PMHNP $P^{3,4}$ \\ ${ }^{1}$ University of Texas Medical Branch, Galveston, TX \\ ${ }^{2}$ Denver Health, Denver, CO \\ ${ }^{3}$ Medical University of South Carolina \\ ${ }^{4}$ University of Colorado in Denver, CO
}

ABSTRACT: Introduction: There is no well-established model in outpatient psychiatry. While there are likely effective models being used in practice, the objective here is to identify the components of an effective outpatient psychiatry model. Obesity contributes to depression and is more prevalent in the mentally ill population. The Chronic Care Model (CCM) has been shown to be a useful framework for promoting weight loss in the severe mentally ill (SMI) population. The collaboration between nurse practitioners and physicians has been shown to be more effective than physician-only practice. With a declining proportion of physicians to 
patients, more effective ways of managing mental health must be a priority.

HYPOTHESIS: The CCM framework can be utilized to develop a team model for outpatient mental health that is more effective and efficient, with an emphasis on promoting overall physical and mental well-being through exercise.

OBJECTIVE: This presentation aims to encourage the development and implementation of team models in psychiatry with particular emphasis on exercise as a centrally integrated component.

METHODS: The CCM was used as a general framework for developing a team model where members have defined roles and tasks. A nurse practitioner and physician team model was used for psychiatric management. The roles of individual team members were evaluated and defined to distribute responsibilities equitably within the team. Weight loss management has been given a prominent role in the overall team model.

RESULTS: The team model roles defined include one psychiatrist, two psychiatric-mental health nurse practitioners (PMHNP), one registered nurse (RN), one certified medical assistant (CMA), one registered dietitian (RD), and one fitness coach.

DISCUSSION: The focus on exercise, as a central component of the outpatient mental health model, promotes both physical and mental health in a population with higher rates of obesity and medical comorbidity. Utilizing both physicians and nurse practitioners reinforces communication and collaboration. Combining a focus on weight management, under the CCM, is presented here as a more effective and efficient outpatient mental health model.

CONCLUSIONS: The team model, developed here under the CCM, has promise for being a standard for outpatient mental health, providing a holistic approach for treating the mentally ill.

FUNDING: There has been no funding for this presentation.

\section{Complex Sleep Behaviors in Patient Treated with Quetiapine and Zolpidem for Insomnia}

Clarice Chan, $M D^{\prime}$; Abhishek Rai, $M D^{\prime}$ and David Leavitt, $M D^{2}$

${ }^{1}$ St. Mary Mercy Hospital, Livonia, MI

${ }^{2}$ St. Joseph Mercy Hospital, Ypsilanti, MI

INTRODUCTION: Complex sleep behaviors like somniloquy (sleep-talking), somnambulism (sleep-walking), sleep-eating and sleep-driving are different forms of parasomnia when patients have these actions during sleep without awareness. Zolpidem is a GABA-A receptor agonist to treat insomnia. Many cases of zolpidem-induced complex sleep behaviors have been reported (Paulke et al. 2014). Quetiapine, an antipsychotic medication, is also widely used off-label to treat insomnia for its antagonistic action on the histamine $\mathrm{H} 1$ and serotonin $2 \mathrm{~A}$ receptors. Evidence supporting its efficacy and safety in treating insomnia is lacking (Anderson \& Griend 2014). A research of literature (PUBMED, June 2014) did not reveal any case demonstrating somniloquy, sleep-eating or sleep-driving associated with quetiapine use. A few cases of somnambulism induced by quetiapine have been reported (Raja \& Raja 2012, Hafeez \& Kalinowski 2007, Seeman 2010). Literature search also did not reveal any report of complex sleep behaviors for zolpidem in combination with quetiapine. One case of somnambulism has been reported for zolpidem when used with valproic acid (Sattar et al. 2003).

CASE PRESENTATION: Patient is a female with bipolar I disorder who was on zolpidem for insomnia for many years without parasomnia. Zolpidem was switched to quetiapine during one hospitalization, and no parasomnia was noted. Upon discharge, zolpidem was added to quetiapine for persistent insomnia. Within 2 months, patient's family expressed concerns about her abnormal nocturnal behaviors: she would arouse from sleep, walk to the kitchen to eat or talk to her friends on the phone for hours. She also had episodes of driving out to buy groceries. There was no recollection of events after. After discontinuation of zolpidem, these behaviors resolved. Patient then had a trial of clonazepam and quetiapine: her insomnia resolved without any recurrence of parasomnia.

DISCUSSION: In the case we illustrated, patient did not develop any parasomnia when she was on zolpidem only, quetiapine only or a combination of quetiapine and clonazepam. She presented with parasomnia on a combination of quetiapine and zolpidem. Most of these behaviors were harmless, though the aberrant nature of them led to substantial distress on patient and family. The etiology is not well understood. According to the serotonergic hypothesis of somnambulism (Juszczak \& Swiergiel 2005), serotonergic neurons can be activated, exert excitatory action on motor neurons and precipitate somnambulism.

CONCLUSION: Majority of psychiatric patients have insomnia and frequently rely on medications like zolpidem and quetiapine to treat it. Complex sleep behaviors have the potential of inflicting harm on the patients and others without any awareness. Legal liability 
becomes a concern, and the case we present in this report thus serves to compel clinicians to consider the possible side effect of parasomnia when they try to treat insomnia.

\section{Case Series Demonstrating Risk of Suicidality with Antiepileptic Drugs - Suicide Attempt After 4 Days of Primidone and 1 Day of Levetiracetam Use}

Ashwini Gulwadi, $M D^{\prime}$; Gen Shinozaki, MD, FAPM'; Kalappurackal $C$ Joseph, $M D^{\prime}$ and Pankaj Lamba, $M D^{\prime}$

${ }^{1}$ St. Mary Mercy Hospital, Livonia, MI

${ }^{2}$ Roy J and Lucille A Carver College of Medicine, University of Iowa Hospitals and Clinics, Iowa City, IA

ABSTRACT: Introduction: In January 2008, FDA issued an alert that antiepileptic drugs (AEDs) might increase risk for suicidality based on pooled analysis of 199 clinical trials of 11 (AEDs) as early as one week after starting treatment. But, many subsequent publications demonstrated several confounding and contradicting factors. The result is a not enough convincing support for the FDA conclusions. Here we present two cases of suicidality and their correlation with AEDs.

CASE DESCRIPTIONS: Case 1 was a 79 -year-old male admitted after a serious suicide attempt. He reported that "earlier that day, I snapped and took about 30 pills starting with "p". As nothing happened, I cut my wrist twice with a knife and waited, hoping to die." His brother found him half-an-hour after the event. He was started on primidone for tremor four days prior and reported feeling increasingly irritable for the past two days. Primidone was discontinued on admission and he continued to feel non-suicidal and showed a broad affect. Case 2 was a 76- year-old female with long history of epilepsy who was admitted after suicide attempt by cutting her both wrists deeply to the extent requiring tendon repair. She reported that she felt "it was the right thing to do" at the moment when she felt acutely depressed after reading about her medication list and side effects list that she got from her pharmacy. Her sonin-law found her four hours after the attempt. She was started on levetiracetam one day ago; prior to that she was on phenytoin for over 30 years for epilepsy. One day after the event, she reported complete resolution of suicidal ideation, and reported better mood. Both cases have no previously diagnosed psychiatric problems and no prior suicide behaviors.

DISCUSSION: Both these cases point towards the suicidal behavior associated with starting AEDs within a week of starting the drug. In addition, in our first case the indication of primidone use was tremors, which unlike epilepsy, is not an independent risk factor for increased suicidality. The potential pathogenic mechanisms remain unclear at present. One of the proposed hypotheses is AEDs with GABAergic properties like barbiturate can increase depression and suicidal behavior through GABAergic - mediated decrease in serotonin at the ralphe nuclei.

CONCLUSION: This is an addition to the sparse clinical literature in regard to the risk of suicidality with AEDs. We propose that the clinicians need to be cognizant of this risk and discuss this with patients and their families prior to starting AEDs.

\section{Comparative Neuropharmacology of Therapeutic Agents Targeting Posttraumatic Stress Disorder}

Bruce Daugherty, PhD'; Gregory Sullivan, $M D^{\prime}$; Leland Gershell, $\mathrm{MD} \mathrm{PhD}^{\prime}$ and Seth Lederman, $\mathrm{MD}^{\prime}$

${ }^{1}$ Tonix Pharmceuticals, Inc., New York, NY

ABSTRACT: Background: Syndromal sleep disturbances in PTSD are targeted by drugs affecting serotonin (5-HT) receptors, particularly 5-HT2A and 5-HT2C. Cyclobenzaprine (CBP) and trazodone (TZD) are bedtime PTSD treatment candidates with several 5-HT actions. The 5-HT receptor activities of CBP and TZD, and their respective metabolites norcyclobenzaprine (nCBP) and meta-chlorophenylpiperazine (mCPP), were analyzed.

METHODS: Binding and functional activities of CBP, nCBP, TZD and mCPP were compared on human 5-HT receptors and transporter (5-HTTP).

RESULTS: For CBP, binding affinities (Ki) were high for 5-HT2A (5.4 nM) and 5-HT2B (15 nM); and moderate for 5-HT2C (43 nM) and 5-HTTP (29 nM). For nCBP, Ki was high for 5-HT2B (12 nM); moderate for 5-HT2A (38 nM) and 5-HT2C (43 nM); and low for 5-HTTP (461 nM). For TZD, Ki was high for 5-HT2A (11 nM); and low for 5HT2B (58 nM), 5-HT2C (250 nM) and 5-HTTP (150 $\mathrm{nM})$. For mCPP, Ki was high for 5-HT2C (9.7 nM); moderate for 5-HT2B (32 nM); and low for 5-HT2A (160 $\mathrm{nM})$. CBP, nCBP and TZD are functional antagonists at 5-HT2A (EC50 230, 140, 470, $3000 \mathrm{nM}$ ), 5-HT2B (EC50 $100,580,3000 \mathrm{nM}$ ) and 5-HT2C (EC50 444, 1220, "Not Active" $\mathrm{nM})$. Whereas, $\mathrm{mCPP}$ is a functional agonist at 5-HT2B (EC50 $6.2 \mathrm{nM}$ ) and 5-HT2C (EC50 $75 \mathrm{nM}$ ).

CONCLUSIONS: CBP, nCBP, and TZD are antagonists at 5HT2B and 5-HT2C. In contrast, mCPP is an agonist, consistent with its described anxiogenic effects in PTSD. Together, these data suggest CBP and its metabolite have similar 5-HT system-mediated sleep-promoting and 
anxiolytic effects that deserve further investigation as a potential bedtime treatment for PTSD.

\section{An Evaluation of the Efficacy of a Low Dose, Bedtime, Sublingual Formulation of Cyclobenzaprine (TNX-102 SL*) in Military- Related PTSD}

Gregory M. Sullivan, MD'; Judy F. Gendreau'; R. Michael Gendreau'; Amy Schaberg ${ }^{3}$; Bruce L. Daugherty'; Heather Jividen'; Ashild Peters'; Perry Peters' ${ }^{\text {and Seth Lederman' }}$

${ }^{1}$ Tonix Pharmaceuticals, Inc., New York, NY

${ }^{2}$ Gendreau Consulting, Poway, CA

${ }^{3}$ Schaberg Consulting, Cary, NC

ABSTRACT: Background: Currently there is an urgent unmet need for evidence based pharmacotherapy interventions for military-related PTSD. As a multifunctional agent with serotonin-2A, alpha-1-adrenergic, and histamine-1 receptor blocking properties, TNX-102 $\mathrm{SL}^{*}$ is designed to target sleep disturbance and hyperarousal in PTSD for the purpose of significantly improving global symptoms of the condition. Therefore, the 'AtEase Study' (TNX-CY-P201) is being conducted to assess the potential clinical benefit of TNX-102 SL in the treatment of military related PTSD.

METHODS: In this multi-center, double-blind, randomized, placebo-controlled, efficacy and safety trial of TNX-102 SL, individuals with military-related PTSD are being recruited by advertisement to test a proprietary low dose sublingual formulation of cyclobenzaprine (TNX-102 SL tablet, Tonix Pharmaceuticals, Inc.) for the treatment of PTSD. Eligible participants (males and females) are 18-65 years of age, have experienced DSM-5 PTSD Criterion A-qualifying trauma(s) during military service since 2001, meet DSM-5-defined PTSD by
CAPS-5 (with severity score $\geq 29$ ), and are free of antidepressants and free of or can be washed off of other psychotropic medications. A total of 220 participants are being randomized to one of three arms for 12-weeks of daily bedtime treatment with: placebo $(\mathrm{N}=88)$, TNX-102 SL $2.8 \mathrm{mg}(\mathrm{N}=88)$, TNX-102SL $5.6 \mathrm{mg}$ $(\mathrm{N}=44)$. Twenty-three private clinical trial sites and three VA sites across the US are administering the trial. All who complete the 12-weeks of the AtEase trial are eligible for an open-label extension study (TNX-CYP202) of 12 additional weeks receiving TNX-102 SL 2.8 $\mathrm{mg}$ daily. The primary efficacy endpoint is mean change from baseline in CAPS-5 score after 12 weeks of treatment between the placebo and TNX-102 SL $2.8 \mathrm{mg}$ groups. Secondary efficacy endpoints include comparisons between all three groups on mean change from baseline in sleep measures and hyperarousal symptoms. Sample size was determined for a power of $80 \%$ to detect an observed effect size of 0.5 (approximately a 10-point difference between the Placebo and the TNX-102 SL groups on the CAPS-5), which is considered a clinically relevant difference.

RESULTS: Recruitment is currently ongoing. Topline results are anticipated in the first half of 2016.

CONCLUSION: The AtEase Study is an ongoing, FDAregistration quality, randomized controlled trial and is the first study to evaluate the clinical utility ofTNX-102 SL taken daily at bedtime as a potential pharmacotherapy for the treatment of military related PTSD. Treatment effects on sleep disturbance and hyperarousal symptoms will be considered in the analysis of the results.

Trial Registration: ClinicalTrials.gov Identifier NCT02277704

*TNX-102 SL is an Investigational New Drug and has not been approved for any indications. 